\title{
Synthetic and therapeutic applications of ammonia-lyases and aminomutases
}

Fabio Parmeggiani, Nicholas J. Weise, Syed T. Ahmed and Nicholas J. Turner*

\author{
School of Chemistry, Manchester Institute of Biotechnology (MIB), University of \\ Manchester, 131 Princess Street, M1 7DN, Manchester, United Kingdom. \\ E-mail: nicholas.turner@manchester.ac.uk
}

\begin{abstract}
$\underline{\text { Abstract }}$
Ammonia-lyases and aminomutases are mechanistically and structurally diverse enzymes which catalyze the deamination and/or isomerization of amino acids in nature by cleaving or shifting a $\mathrm{C}-\mathrm{N}$ bond. Of the many protein families in which these enzyme activities are found, only a subset have been employed in the synthesis of optically pure fine chemicals or in medical applications. This review covers the natural diversity of these enzymes, highlighting particular enzyme classes that are used within industrial and medical biotechnology. These highlights detail the discovery and mechanistic investigations of these commercially-relevant enzymes, along with comparisons of their various applications as stand-alone catalysts, components of artificial biosynthetic pathways and biocatalytic or chemo-enzymatic cascades, and therapeutic tools for the potential treatment of various pathologies.
\end{abstract}




\section{Contents}

1. Introduction

1.1 Classification of ammonia-lyases

1.1.1 Aspartate ammonia-lyases

1.1.2 Methylaspartate ammonia-lyases

1.1.3 Aminoacyl-CoA ammonia-lyases

1.1.4 Aromatic amino acid ammonia-lyases

1.1.5 Hydroxy amino acid dehydratases/deaminases

1.1.6 Ethanolamine ammonia-lyases

1.1.7 Amino acid cyclodeaminases

1.2 Classification of aminomutases

1.2.1 AdoCbl-dependent aminomutases

1.2.2 SAM-dependent aminomutases

1.2.3 MIO-dependent aminomutases

1.2.4 PLP-dependent aminomutases/aminotransferases

1.3 Scope of this review

2. Structure and mechanism of aspartate and methylaspartate ammonia-lyases

2.1 Aspartate ammonia-lyases (DALs)

2.2 Methylaspartate ammonia-lyases (MALs)

3. Structure and mechanism of aromatic amino acid ammonia-lyases and aminomutases

3.1 Arylalanine ammonia-lyases (PALs, HALs and TALs)

3.2 Arylalanine aminomutases (PAMs and TAMs)

4. Synthetic applications of ammonia-lyases and aminomutases

4.1 Synthesis of aspartic acid derivatives

4.2 Synthesis of L-arylalanines

4.3 Synthesis of D-arylalanines

4.4 Synthesis of $\beta$-arylalanines

4.5 Enzymatic and chemo-enzymatic cascade applications

5. Therapeutic applications of ammonia-lyases

6. Conclusions and perspectives 


\section{Introduction}

During the past decade, biocatalysis has had an increasing impact on manufacturing in the chemical industry, leading to the development of greener, more efficient and more sustainable synthetic processes. This impact has been especially prominent in the preparation of pharmaceuticals, agrochemicals and fine chemicals, as well as in the development of new biopharmaceuticals for the treatment of several diseases. However, even though the industrialscale use of enzymes is now well established, its application is somewhat limited to specific enzyme classes (e.g., lipases, proteases, dehydrogenases and more recently transaminases, aldolases, nitrilases, etc.). At the research level, biocatalysis is a rapidly expanding area that offers novel catalysts, processes and technologies at an ever-accelerating pace. Some of these new biocatalysts present great potential for stereoselective synthesis, but are currently not applied in a general sense to large scale manufacturing.

The enzymes belonging to the structurally and mechanistically diverse families of ammonialyases and aminomutases exemplify this situation perfectly. These proteins catalyze a range of transformations based upon $\alpha$ - and $\beta$-amino acid scaffolds (and rarely upon other amine derivatives), with excellent stereoselectivity and often perfect atom economy. In common with many other classes of biocatalysts, the toolbox of ammonia-lyases and aminomutases is rapidly expanding as a result of recent developments in bioinformatics, protein engineering and directed evolution, which have led to a significant increase in the number and range of enzymes available for screening and application in organic synthesis. Furthermore, most often the reactions catalyzed by these enzyme families do not have a counterpart in traditional synthetic organic chemistry. This makes their implementation in synthetic processes highly desirable for preparative applications at any scale, but specifically with a view to industrial production of value-added chemicals that are hard to access stereoselectively otherwise. 


\subsection{Classification of ammonia-lyases}

Ammonia-lyases (EC 4.3.1.X, defined as carbon-nitrogen lyases that release ammonia as one of the products) catalyze the reversible cleavage of $\mathrm{C}-\mathrm{N}$ bonds, typically of $\alpha$-amino acids, generating ammonia and an unsaturated or cyclic derivative. In spite of this apparently specific and well-defined transformation, the differences in structure, function and mechanism are remarkable, giving rise to 31 different EC subclasses (Table 1). The vast majority of these enzymes (definitely all the most relevant and better studied subclasses) fall into one of seven main groups based on the specific analogies in the reaction they catalyze and the types of substrates they accept (Scheme 1).

1.1.1 Aspartate ammonia-lyases. Aspartate ammonia-lyases (DALs), also known as aspartases, catalyze the reversible deamination of L-aspartic acid to fumaric acid. ${ }^{1-3}$ They play a crucial role in bacterial nitrogen metabolism and they belong to a broad and well-characterized superfamily (the "aspartase/fumarase" superfamily) with a characteristic tertiary and quaternary structure, as well as a similar active site architecture. ${ }^{4}$ However, other members of this superfamily are known to catalyze different $\mathrm{C}-\mathrm{N}$ lyase reactions, $\mathrm{C}-\mathrm{O}$ lyase reactions and other unrelated transformations. The vast majority of the practical applications of aspartase exploit the reverse of the natural reaction for the asymmetric synthesis of L-aspartic acid. Aspartase is among the most specific enzymes known, with multiple attempts of engineering failing to afford more versatile biocatalysts. However, the synthesis of a limited range of aspartic acid analogues with aspartases has been demonstrated. Section 2.1 will deal with the structure and properties of DALs, while their applications will be presented in Section 4.1. 
Table 1. Updated EC classification of ammonia-lyases (obsolete classes are shown in red).

\begin{tabular}{|c|c|c|c|c|}
\hline EC number & Enzyme & Acronym & Created & Last modified \\
\hline 4.3 .1 .1 & aspartate ammonia-lyase & DAL & 1961 & \\
\hline 4.3.1.2 & methylaspartate ammonia-lyase & MAL & 1961 & \\
\hline 4.3.1.3 & histidine ammonia-lyase & HAL & 1961 & 2008 \\
\hline 4.3.1.4 & formimidoyltetrahydrofolate cyclodeaminase & & 1961 & 2000 \\
\hline 4.3.1.5 & $\begin{array}{l}\text { phenylalanine ammonia-lyase } \\
\text { (deleted and subdivided into EC 4.3.1.23-25) }\end{array}$ & PAL & 1965 & deleted 2008 \\
\hline 4.3.1.6 & $\beta$-alanyl-CoA ammonia-lyase & $\beta A C A L$ & 1965 & \\
\hline 4.3.1.7 & ethanolamine ammonia-lyase & EAAL & 1972 & \\
\hline 4.3.1.8 & $\begin{array}{l}\text { hydroxymethylbilane synthase } \\
\text { (transferred to EC 2.5.1.61) }\end{array}$ & & 1972 & deleted 2003 \\
\hline 4.3.1.9 & D-glucosaminate ammonia-lyase & DGDD & 1972 & 2004 \\
\hline 4.3.1.10 & L-serine- $O$-sulfate ammonia-lyase & & 1972 & \\
\hline 4.3.1.11 & $\begin{array}{l}\text { dihydroxyphenylalanine ammonia-lyase } \\
\text { (deleted for lack of experimental evidence) }\end{array}$ & & 1972 & deleted 2007 \\
\hline 4.3.1.12 & ornithine cyclodeaminase & OCD & 1976 & \\
\hline 4.3.1.13 & carbamoylserine ammonia-lyase & & 1976 & \\
\hline 4.3.1.14 & 3-aminobutyryl-CoA ammonia-lyase & ABCAL & 1999 & \\
\hline 4.3.1.15 & diaminopropionate ammonia-lyase & & 1999 & \\
\hline 4.3.1.16 & threo-3-hydroxy-L-aspartate ammonia-lyase & & 2001 & 2011 \\
\hline 4.3.1.17 & L-serine ammonia-lyase & LSDD & 2001 & 2014 \\
\hline 4.3.1.18 & D-serine ammonia-lyase & DSDD & 2001 & \\
\hline 4.3.1.19 & threonine ammonia-lyase & TDD & 2001 & 2014 \\
\hline 4.3.1.20 & erythro-3-hydroxy-L-aspartate ammonia-lyase & & 2001 & 2011 \\
\hline 4.3.1.21 & $\begin{array}{l}\text { aminodeoxygluconate ammonia-lyase } \\
\text { (deleted, identical to EC 4.3.1.9) }\end{array}$ & & 2002 & deleted 2004 \\
\hline 4.3.1.22 & 3,4-dihydroxyphenylalanine reductive deaminase & & 2007 & \\
\hline 4.3.1.23 & tyrosine ammonia-lyase & TAL & 2008 & \\
\hline 4.3.1.24 & phenylalanine ammonia-lyase & PAL & 2008 & \\
\hline 4.3.1.25 & phenylalanine/tyrosine ammonia-lyase & PAL/TAL & 2008 & \\
\hline 4.3.1.26 & $\begin{array}{l}\text { chromopyrrolate synthase } \\
\text { (transferred to EC 1.21.3.9) }\end{array}$ & & 2010 & deleted 2013 \\
\hline 4.3.1.27 & threo-3-hydroxy-D-aspartate ammonia-lyase & & 2011 & \\
\hline 4.3.1.28 & L-lysine cyclodeaminase & $\mathrm{KCD}$ & 2012 & \\
\hline 4.3.1.29 & D-glucosaminate-6-phosphate ammonia-lyase & & 2013 & \\
\hline 4.3.1.30 & $\begin{array}{l}\text { dTDP-4-amino-4,6-dideoxy-D-glucose ammonia- } \\
\text { lyase }\end{array}$ & & 2011 & \\
\hline 4.3.1.31 & L-tryptophan ammonia-lyase & & 2016 & \\
\hline
\end{tabular}




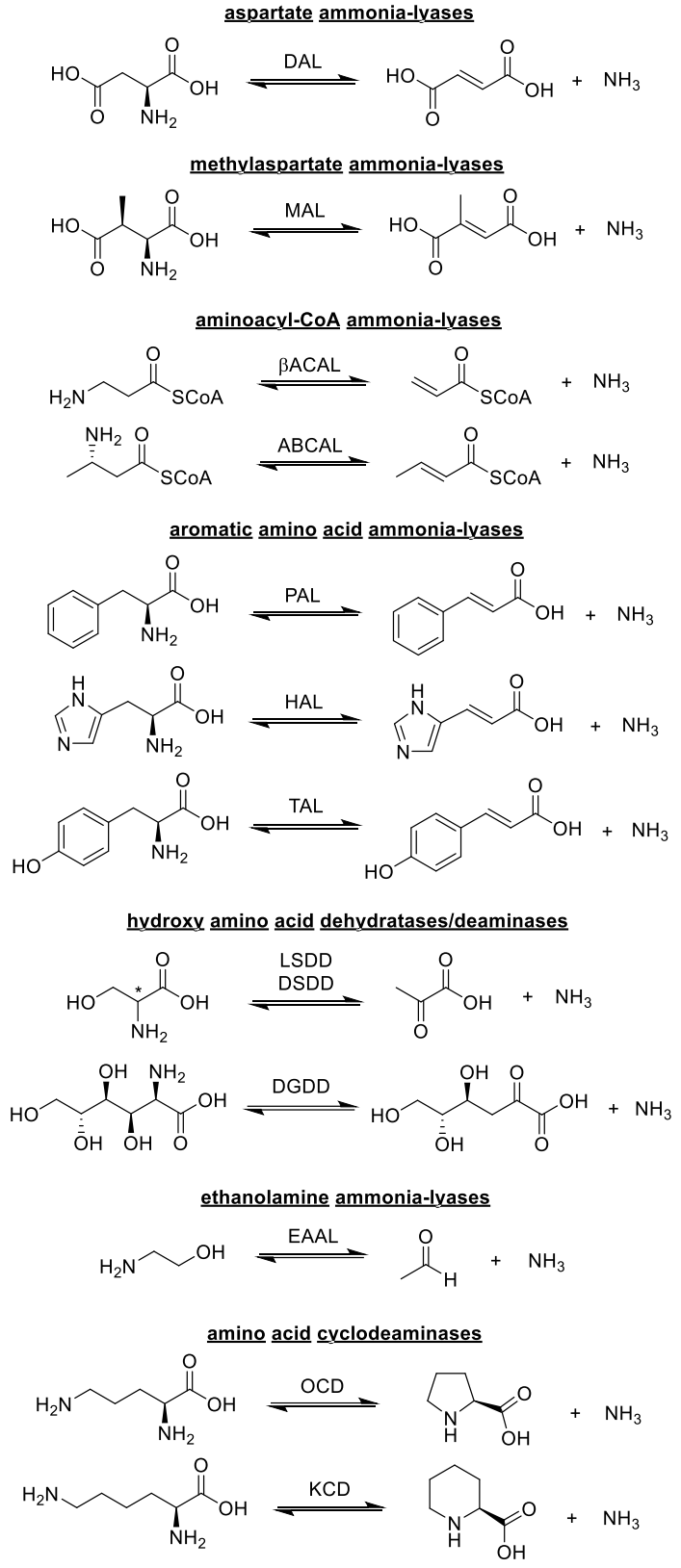

Scheme 1. Representative reactions catalyzed by the most important classes of ammonia-lyases. 


\subsubsection{Methylaspartate ammonia-lyases. 3-Methylaspartate ammonia-lyases, or}

methylaspartases (MALs) share mechanistic and structural features with the enolase superfamily. ${ }^{2,5,6}$ They were first identified in anaerobic bacteria but later proven to be insensitive to oxygen and found in a number of non-anaerobic species. MALs catalyze the deamination of (2S,3S)-threo-3-methylaspartate to 2-methylfumaric acid (i.e., mesaconic acid) and require no specific cofactor, only divalent $\left(\mathrm{Mg}^{2+}\right)$ and monovalent $\left(\mathrm{K}^{+}\right)$cations. In spite of the similarity of the transformation performed, MALs are structurally very different from DALs, being more related to the versatile superfamily of enolases, that catalyze a broad range of reactions (e.g., dehydrations, racemizations and cycloisomerizations). ${ }^{7}$ Also, again in contrast with aspartases, the substrate tolerance of methylaspartases is much broader and it has been expanded further by structure-based protein engineering, leading to the synthesis of a large variety of substituted aspartic acid derivatives. MALs will be discussed in detail in Section 2.2, and their applications in Section 4.1.

1.1.3 Aminoacyl-CoA ammonia-lyases. The fermentation of $\beta$-alanine, performed only by very few organisms, proceeds via activation to the CoA thioester, followed by the elimination of ammonia by a $\beta$-alanyl-CoA ammonia-lyase ( $\beta$ ACAL), producing acryloyl-CoA. Two enzymes of this class have been identified in Clostridium propionicum, and one of them (Cp- $\beta$ ACAL-2) isolated, purified and demonstrated to be active. ${ }^{8}$ The reversibility of the reaction and the tolerance towards different derivatives of the substrate (e.g., crotonyl-CoA, methacryloyl-CoA) have also been demonstrated, illustrating a possible application in the production of small $\beta$ amino acids. More recent structural studies on Cp- $\beta$ ACAL-2, including a crystal structure in complex with propionyl-CoA, led to the proposal of a mechanism based on the formation of an enolate intermediate and retro-Michael elimination of an ammonium ion. ${ }^{9}$ A similar activity is that of L-3-aminobutyryl-CoA ammonia-lyase (ABCAL), first identified in Clostridium subterminale,${ }^{10}$ and later in other organisms such as Brevibacterium. ${ }^{11}$ This enzyme converts 3aminobutyryl-CoA into crotonyl-CoA and ammonia, and it has been postulated to follow the 
same mechanism as $\beta A C A L$. Interestingly, it has been demonstrated that ammonia can be replaced with hydroxylamine in the ABCAL amination reaction, leading to the heterocycle 3methyl-5-isoxazolidinone, after cyclization of the expected 3-hydroxyaminobutyryl-CoA product and loss of CoA. ${ }^{10}$ However, due to the difficulty of accessing CoA-derivatives as substrates, these enzymes are unlikely to find widespread synthetic use in large scale applications.

1.1.4 Aromatic amino acid ammonia-lyases. Phenylalanine ammonia-lyase (PAL), histidine ammonia-lyase (HAL) and tyrosine ammonia-lyase (TAL) catalyze the reversible elimination of ammonia from the corresponding aromatic L-amino acids, yielding cinnamic acid, urocanic acid and $p$-coumaric acid, respectively. ${ }^{12-15}$ Only one report is available in the literature about the conversion of L-tryptophan to 3-indoleacrylic acid by a putative tryptophan ammonia lyase (WAL) from Rubrivivax benzoatilyticus, ${ }^{16}$ however the gene has not been conclusively identified and further work will be necessary before the existence of such enzyme in nature can be confirmed. The characteristic feature of arylalanine ammonia-lyases is the presence of the unusual highly electrophilic heterocycle 4-methylideneimidazol-5-one (MIO), formed by posttranslational cyclization and dehydration of a tripeptide in the active site, which mediates these challenging amination/deamination reactions. ${ }^{14,17}$ This functionality is often improperly called "MIO cofactor", especially in the older literature, but this is not formally correct because cofactors are non-protein-derived molecules (or ions). Similarly, it is often referred to as "MIO prosthetic group", but this is also incorrect, since it implies the separate biosynthesis and subsequent post-translational attachment of this group to the apo-enzyme. Given that the formation of the MIO occurs from nothing but the folded polypeptide chain, it would be better described just as a modified active site residue or catalytic moiety, instead. In recent years, aromatic amino acid ammonia-lyases (particularly PALs) have become popular as excellent and versatile catalysts for the synthesis of substituted amino acids, and a plethora of applications have been described with these enzymes alone or in the context of chemo-enzymatic cascade catalysis. ${ }^{18-21}$ A comprehensive description of the structure and mechanistic studies of arylalanine 
ammonia-lyases will be given in Section 3.1 and their synthetic and biotherapeutic applications will be discussed in Sections 4 and 5, respectively.

1.1.5 Hydroxy amino acid dehydratase/deaminases. A large family of phylogenetically related enzymes dependent on pyridoxal 5'-phosphate (PLP) catalyze reversible reactions where the C-N bond of a $\beta$-hydroxy- $\alpha$-amino acid is replaced with a $\mathrm{C}=\mathrm{O}$ bond, with concomitant elimination of ammonia and water. These enzymes belong to class II of the PLP-dependent enzymes family, and their mechanism involves binding to PLP as an imine (through the $\alpha$-amino group) and elimination of water from the $\beta$-position of the substrate, yielding an enamine that tautomerizes to the imine and is rapidly hydrolyzed non-enzymatically in the aqueous environment, yielding the corresponding $\alpha$-keto acid and ammonia. These enzymes are often referred to as ammonia-lyases because of the $\mathrm{C}-\mathrm{N}$ bond cleavage, but naming them dehydratases/deaminases reflects more clearly the steps of the mechanism (noting that a water molecule is lost in the first step, but one is required for imine hydrolysis). Examples are L- or D-serine dehydratase/deaminase (L- or Dserine to pyruvate), ${ }^{22,23}$ L-threonine dehydratase/deaminase (L-threonine to 2-oxoglutarate), ${ }^{24,25}$ L-threo-3-hydroxyaspartate dehydratase/deaminase (L-threo-3-hydroxyaspartate to oxaloacetate $^{26}$ and D-glucosaminate dehydratase/deaminase (D-glucosaminate to D-2-dehydro3-deoxygluconate). ${ }^{27}$ One interesting exception is diaminopropionate ammonia-lyase (L- $\alpha, \beta$ diaminopropionate to pyruvate), ${ }^{28}$ since its substrate bears an amino group instead of a hydroxyl group at the $\beta$-position, and therefore the $\alpha, \beta$-elimination results in a loss of ammonia instead of water. Another related exception is carbamoylserine ammonia-lyase, that cleaves the carbonoxygen bond of carbamoylserine, releasing $\mathrm{CO}_{2}$ and a first molecule of ammonia, followed by the usual tautomerization and hydrolytic deamination to form pyruvate with elimination of a second molecule of ammonia. ${ }^{29}$ No preparative application of these enzymes is known, even though a few have been used in the design of assays or biosensors. ${ }^{30,31}$

1.1.6 Ethanolamine ammonia-lyases. Even though the overall reaction of ethanolamine ammonia-lyase (EAAL) resembles closely that of dehydratases/deaminases, this enzyme 
performs the conversion with a completely different mechanistic pathway. EAAL reversibly converts ethanolamine into acetaldehyde and ammonia, an activity first discovered in cholinefermenting Clostridium sp. $^{32}$ and later identified in several other organisms, including Salmonella, Klebsiella and Bacillus. Unlike any other ammonia-lyase, EAALs require the cofactor adenosylcobalamin (AdoCbl or coenzyme $\left.\mathrm{B}_{12}\right){ }^{33}$ The availability of crystal structures of the enzyme with cofactor analogues bound ${ }^{34}$ supported mechanistic studies and gave a precise view of the key binding interactions. The mechanism has been extensively studied with native and recombinant enzymes and found to be more similar to that of AdoCbl-dependent aminomutases (see Section 1.2.1). The Co-C bond of AdoCbl is cleaved homolytically, forming the 5'-deoxyadenosyl free radical, which removes a proton radical from $\mathrm{C} 1$ of the substrate. The ethanolamine-derived radical thus generated undergoes a rearrangement with migration of the amine from $\mathrm{C} 2$ to $\mathrm{C} 1$, yielding a hemiaminal radical, which abstracts a hydrogen radical from 5'deoxyadenosine, to regenerate the 5'-deoxyadenosyl radical. The resulting hemiaminal rapidly decomposes to the products ammonia and acetaldehyde. ${ }^{35,36}$ In spite of the very extensive body of work on the mechanistic aspects of these fascinating enzymes, alternative substrates have not been found and the applications of EAALs remain rather limited.

1.1.7 Amino acid cyclodeaminases. The first member of this class to be identified and isolated was L-ornithine cyclodeaminase (OCD) from Clostridium sporogenes PA 3679, which converts L-ornithine to L-proline and ammonia. ${ }^{37}$ Early mechanistic studies suggested that the reaction involves the initial deamination of the $\alpha$-amino group of the substrate to give 2-oxo-5aminopentanoate, which cyclizes spontaneously to $\Delta^{1}$-pyrroline-2-carboxylate, followed by reduction of the latter to L-proline. ${ }^{38}$ These two complementary redox reactions are carried out by a tightly bound nicotinamide adenine dinucleotide $\left(\mathrm{NAD}^{+}\right)$cofactor molecule in the active site, via hydride abstraction and donation. Thus, amino acid cyclodeaminases are the only nicotinamide cofactor-dependent ammonia-lyases known to date. As a matter of fact, this mechanism resembles closely that of amino acid dehydrogenases (classified as oxidoreductases); 
however, the cyclization/reduction steps make the whole process redox-neutral, thus cyclodeaminases are better regarded to as ammonia-lyases. The first X-ray crystal structure of an OCD (from Pseudomonas putida) ${ }^{39}$ allowed the definition of substrate binding residues and the proposal of an alternative mechanism, in which the imine formed by dehydrogenation of the substrate undergoes attack by the free amine to give an $\alpha$-aminoproline intermediate, from which ammonia is eliminated. The resulting imine is then reduced as before. In a subsequent attempt to elucidate the biosynthesis of pipecolate-containing macrolide immunosuppressants such as rapamycin, the analogous cyclodeamination reaction of L-lysine to L-pipecolic acid was also identified. ${ }^{40,41}$ This reaction is catalyzed by L-lysine cyclodeaminases (KCDs), with strong mechanistic similarities to OCDs. ${ }^{42}$ Recombinant expression and purification of a few cyclodeaminases has been achieved, opening up the way to practical applications. For instance, very recently, a simple and highly efficient process for the conversion of L-lysine to L-pipecolic acid was demonstrated using whole cells of $E$. coli overproducing KCD as the biocatalyst, up to a 17.25 $\mathrm{g} \mathrm{L}^{-1}$ product concentration. ${ }^{43}$ Another example is the synthetic biology approach to the production of L-proline by expression of the ocd gene from $P$. putida in an ornithine-producing Corynebacterium glutamicum strain, with yields of up to $0.36 \mathrm{~g}$ L-proline per gram of glucose. ${ }^{44}$ These results already showed the great synthetic potential of cyclodeaminases, and intensification of these processes could offer significantly improved industrial routes to cyclic amino acids in the years to come.

\subsection{Classification of aminomutases}

Aminomutases (EC 5.4.3.X, defined as isomerases mediating intramolecular transfer of amino groups) catalyze the synthetically challenging shift of an amine group along a saturated carbon chain, typically of an amino acid. It must be noted that such a rearrangement is normally described as a "1,2-shift" since the amino group is transferred between two adjacent carbon atoms, but this is not meant to indicate that such atoms are necessarily the first two in the chain. 
Enzymes with aminomutase activity are widespread and involved in the synthesis of diverse natural products in many different organisms (11 distinct EC subclasses identified at present, listed in Table 2). ${ }^{45,46}$ Due to the range of proteinogenic amino acids accepted by different classes of aminomutases, they employ a broad variety of chemistries depending on the substrate type. In fact, in order to achieve the common goal of shifting an amino group, nature has been observed to select and adapt different catalytic strategies, which play different roles in the synthesis of complex natural products and the anaerobic degradation of amino acids. The distant phylogenetic relationships and very different roles make a unified description of aminomutases impossible, even to a greater extent than for ammonia-lyases. Nonetheless, based on the mechanism and cofactor dependency, it is convenient to classify them into four main families (Scheme 2).

Table 2. Updated EC classification of aminomutases (obsolete classes are shown in red).

\begin{tabular}{lllll}
\hline EC number & Enzyme & Acronym & Created & Last modified \\
\hline 5.4.3.1 & $\begin{array}{l}\text { ornithine 4,5-aminomutase } \\
\text { (deleted, multiple enzymes responsible) }\end{array}$ & & 1972 & deleted 1976 \\
5.4.3.2 & lysine 2,3-aminomutase & $2,3-\mathrm{KAM}$ & 1972 & \\
5.4 .3 .3 & B-lysine 5,6-aminomutase & $5,6-\mathrm{KAM}$ & 1972 & \\
5.4 .3 .4 & D-lysine 5,6-aminomutase & 1972 & 2003 \\
5.4 .3 .5 & D-ornithine 4,5-aminomutase & $4,5-\mathrm{DOAM}$ & 1972 & \\
5.4 .3 .6 & tyrosine 2,3-aminomutase & TAM & 1976 & \\
5.4 .3 .7 & leucine 2,3-aminomutase & & 1982 & \\
5.4 .3 .8 & glutamate-1-semialdehyde 2,1-aminomutase & GSAM & 1983 & \\
5.4 .3 .9 & glutamate 2,3-aminomutase & EAM & 2012 & \\
5.4 .3 .10 & phenylalanine aminomutase (L- $\beta$-Phe forming) & $(S)$-PAM & 2013 & \\
5.4 .3 .11 & phenylalanine aminomutase (D- $\beta$-Phe forming) & $(R)$-PAM & 2013 & \\
& & & & \\
\hline
\end{tabular}



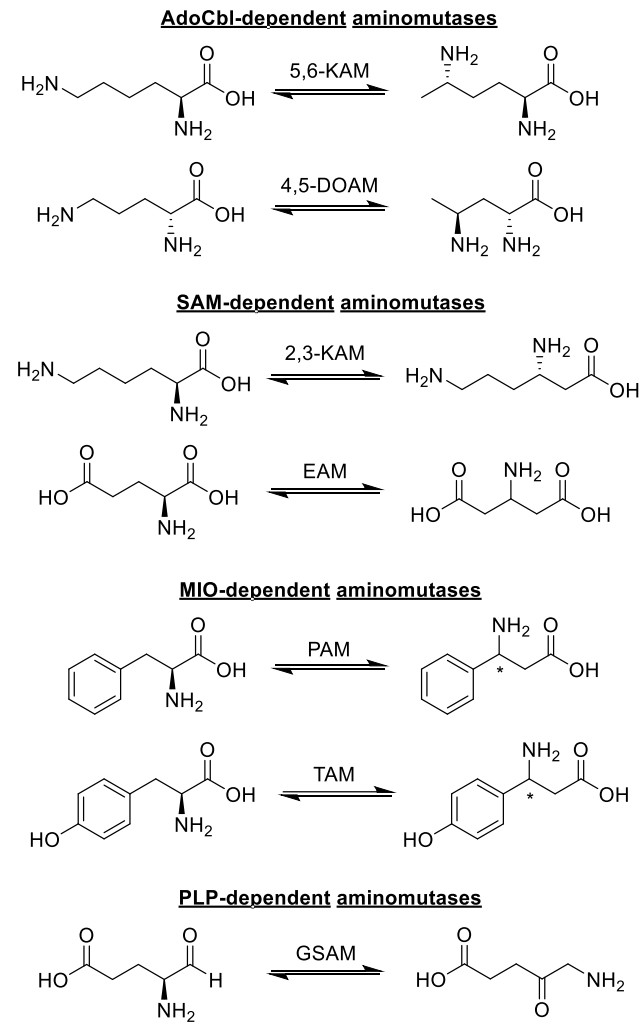

Scheme 2. Representative reactions catalyzed by the most important classes of aminomutases.

1.2.1 AdoCbl-dependent aminomutases. These enzymes catalyze the migration of terminal amino groups to the adjacent position: examples are lysine 5,6-aminomutase (5,6-KAM) and Dornithine 4,5-aminomutase (4,5-DOAM). In order to perform such a complex transformation, they require a radical mechanism using adenosylcobalamin (AdoCbl or coenzyme $\mathrm{B}_{12}$ ) and pyridoxal 5'-phosphate (PLP) as cofactors. The key steps of the mechanism are the homolytic cleavage of the Co-C bond of AdoCbl, forming the 5'-deoxyadenosyl free radical, the abstraction of a hydrogen atom from the substrate (stabilized in the active site by PLP), isomerization via an aziridinecarbinyl radical intermediate, and finally radical transfer to 5'-deoxyadenosine to regenerate the 5'-deoxyadenosyl radical. This has been shown to be associated with large conformational changes bringing the PLP-bound substrate and the AdoCbl close to each other only after substrate binding. ${ }^{47,48,49}$ It has been suggested that such a "locking" mechanism 
prevents side reactions involving the highly reactive radicals formed next to the AdoCbl center. The first AdoCbl-dependent 5,6-KAM and 4,5-DOAM were isolated in the 1970s from the anaerobic bacterium Clostridium sticklandii, ${ }^{50,51}$ and since then several enzymes of this class have been successfully produced in E. coli and purified. ${ }^{52}$ The recombinant proteins showed reasonable activity/stability and, most importantly, are not sensitive to oxygen. In spite of these advantages for the production, purification and use of AdoCbl-dependent mutases, practical applications in biocatalysis are still absent. In particular, one serious issue is light sensitivity (common to all AdoCbl-dependent enzyme, since photolysis of AdoCbl produces hydroxocobalamin that can act as a strong inhibitor).

1.2.2 SAM-dependent aminomutases. The most studied member of this class is lysine 2,3aminomutase (2,3-KAM), discovered in Clostridium subterminale SB4 in the context of lysine metabolism. ${ }^{53,54,55}$ This enzyme mediates the interconversion of L- $\alpha$-lysine to $(S)$ - $\beta$-lysine, and requires three cofactors: $S$-adenosylmethionine (SAM), PLP and a $[4 \mathrm{Fe}-4 \mathrm{~S}]$ cluster ${ }^{56}$ In a similar fashion to AdoCbl-dependent mutases, SAM-dependent mutases require the formation of the 5'deoxyadenosyl radical, but since the homolytic cleavage of the C-S bond of SAM is very difficult, the $[4 \mathrm{Fe}-4 \mathrm{~S}]$ cluster is required as electron donor. ${ }^{57}$ The mechanism is analogous to AdoCbl mutases: the 5'-deoxyadenosyl radical abstracts a hydrogen from the $\beta$-position of the PLP-bound substrate to form a $\beta$-radical intermediate, which rearranges to the more stable $\alpha$ radical via an aziridinyl intermediate. In this species the radical is stabilized by the carboxylic acid group, and its reaction with adenosine to produce the $\beta$-lysine product and regenerate the 5'deoxyadenosyl radical completes the cycle. ${ }^{56,58}$ Other members of this family were discovered more recently, including an enantiocomplementary version of 2,3-KAM from E. coli (forming $(R)$ - $\beta$-lysine, albeit with much lower activity), ${ }^{59}$ and glutamate 2,3 -aminomutases (EAM) from Clostridium difficile $e^{60}$ and thermostable organisms such as Thermoanaerobacter tengcongensis and Morella thermoacetica. ${ }^{61}$ A few very promising applications of these enzymes have been demonstrated in the last decade, such as the evolution of 2,3-KAM into an alanine aminomutase 
for the production of other $\beta$-amino acids including $\beta$-alanine, ${ }^{62,63}$ or the production of $\beta$ glutamate with EAMs. ${ }^{61}$ The potential synthetic use of this class of enzymes is appealing, especially for non-natural substrates, but due to their sensitivity to oxygen and the requirement for expensive cofactors, large scale processes are still not established.

1.2.3 MIO-dependent aminomutases. Remarkably, the same transformation catalyzed by SAMdependent mutases can be performed easily on aromatic amino acids by this class of enzymes, with no requirement for radical sources or oxygen-free conditions. Phenylalanine aminomutase (PAM) and tyrosine aminomutase (TAM) catalyze the 2,3-shift of the $\alpha$-amino group of Lphenylalanine and L-tyrosine to afford $\beta$-phenylalanine and $\beta$-tyrosine, respectively. ${ }^{12,13,45}$ This relatively newly discovered class of mutases is the only case where there are close similarities between ammonia-lyases and aminomutases, since PAMs and TAMs share the same structure, mechanistic pathway and characteristics of PALs, TALs and HALs, being all members of the same MIO-dependent enzyme family (also called "class I lyase-like enzymes"). Another feature of the isomerization reactions catalyzed within this protein family is the production of both $(R)$ and (S)- $\beta$-amino acids from the proteinogenic L- $\alpha$-regioisomers by enzymes with enantiocomplementary selectivity, something uncharacteristic of other aminomutases classes. The availability of stereocomplementary enzymes is of great interest for the development of preparative strategies, considering that both enantiomers of $\beta$-arylalanines are valuable chiral building blocks for synthetic and medicinal chemistry. The features and the applications of this class of mutases will be described in detail in Sections 3.2 and 4.4, respectively.

1.2.4 PLP-dependent aminomutases/aminotransferases. A rather different aminomutase reaction is the 2,1-shift of the amino group of aminoaldehydes (with concomitant 1,2-shift of the carbonyl group) to afford terminal aminoketones. The only example is the isomerization of Lglutamate-1-semialdehyde to 5-aminolevulinate, a precursor of chlorophyll, heme, porphyrins, and other tetrapyrrolic compounds. This reaction is performed in plants and several bacteria by glutamate-1-semialdehyde 2,1-aminomutase (GSAM), a PLP-dependent enzyme first isolated 
from barley leaves. ${ }^{64}$ The reaction involves the exchange of the amino group with a carbonyl functionality (rather than with a proton as seen for all the other aminomutases) and, in fact, it is closely related to that of transaminases. ${ }^{65}$ Indeed, the structure of GSAM is homologous to many PLP-dependent transaminases and the proposed mechanism involves the same PLP-mediated imine formation chemistry, with diaminovalerate as the key intermediate. ${ }^{66-68}$ Due to the poor stability of aminoaldehyde starting materials, the biocatalytic applications of this enzymatic activity are limited, compared to other transaminases.

\subsection{Scope of this review}

Considering this impressive variety of enzyme subclasses, with completely different mechanistic and structural features, a comprehensive report covering all the families mentioned above would be beyond the scope of this review. Several previous accounts and perspectives have already covered in detail the structure and mechanism of specific classes of ammonia-lyases ${ }^{1-3,12-15,19,69-71}$ and aminomutases. ${ }^{45,46,72}$ Nevertheless, since great interest has been devoted to some of these families recently for their (potential or demonstrated) synthetic, industrial or therapeutic applications, the present review intends to cover in detail only these subclasses: aspartate ammonia-lyases (Section 2.1), methylaspartate ammonia-lyases (Section 2.2), arylalanine ammonia-lyases (Section 3.1) and arylalanine aminomutases (Section 3.2), with specific emphasis on their applications (Sections 4 and 5). A few members of these families have been recently demonstrated as valuable tools for the synthesis of high value-added products (e.g., pharmaceuticals, agrochemicals, chiral building blocks) or for biotherapeutic applications in the treatment of specific diseases. 


\section{Structure and mechanism of aspartate and methylaspartate ammonia-lyases}

\subsection{Aspartate ammonia-lyases (DALs)}

The first report of the conversion of L-aspartic acid to succinic acid (a two-step process involving aspartase and a reductase) by Escherichia coli, formerly known as Bacillus coli communis, dates back to $1901,{ }^{73}$ followed by the identification of an equilibrium reaction between L-aspartate, fumarate and ammonia promoted by resting cells of the same organism. ${ }^{74}$ Only in 1971 was the enzyme responsible for this activity (EcDAL, also known as AspA) isolated and purified from $E$. coli. ${ }^{75}$ Even though AspA remains the most studied member of the aspartase family, several other

DALs have been isolated from various sources, for instance Pseudomonas fluorescens, ${ }^{76}$ Hafnia alveis (formerly known as Bacterium cadaveris), ${ }^{77}$ Aeromonas media, ${ }^{78}$ Bacillus subtilis $^{79}$ and Bacillus sp. YM55-1 (also known as AspB). ${ }^{80}$

The first crystal structure of $\mathrm{AspA}^{81}$ showed that the enzyme shares structural features with several other proteins, now collectively referred to as the aspartase/fumarase superfamily. ${ }^{4}$ This family includes not only some related C-N lyases acting on $\mathrm{N}$-substituted derivatives of aspartic acid, such as argininosuccinate lyase (ARL) and adenylosuccinate lyase (ADL), but also the ubiquitous fumarase, also known as fumarate hydratase (FUM), that mediates the reversible hydration of fumarate to L-malate, and other mechanistically related enzymes such as the cis,cismuconate-lactonizing enzyme (CMLE) that converts 3-carboxy-cis,cis-muconate into a muconolactone (Scheme 3). Interestingly, $\delta$-crystallin, the major soluble protein in the eye lenses of birds and reptiles, is also a member of this family due to its close structural relationship with ARL, from which it is thought to have evolved through gene sharing. ${ }^{82}$ Of the two isoforms of $\delta$ crystallin known, only one exhibits ARL activity, but it has been shown through mutagenesis that the other isoform can also regain such activity. 

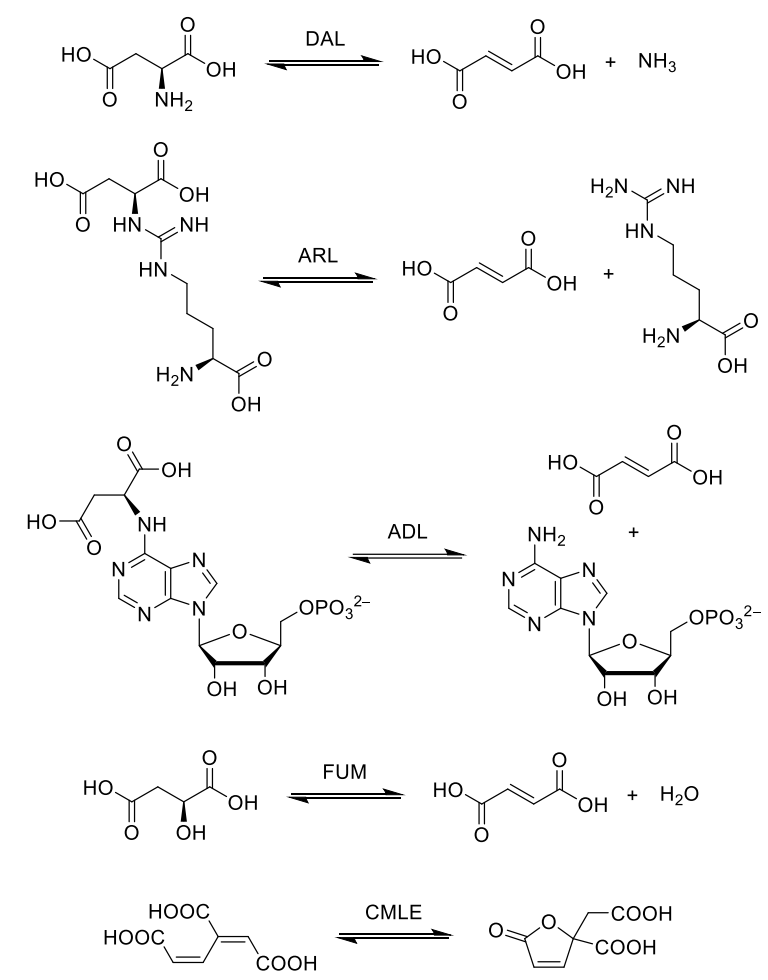

Scheme 3. Reactions catalyzed by members of the aspartase/fumarase superfamily.

The members of the aspartase/fumarase superfamily are homotetramers, with each monomeric subunit being composed of three mainly $\alpha$-helical domains ( $N$-terminal, central, $C$-terminal). The bundles of five rather long $\alpha$-helices in the central domain in each subunit interact with each other forming a tight core of 20 helices in the middle of the tetramer (Figure 1). These features of the tertiary and quaternary structure were first discovered in 1994, with the structural determination of turkey $\delta$-crystallin, ${ }^{83}$ and later found to be common to the whole superfamily. In spite of the very low sequence identities among different members (as low as 15\%), another distinctive feature is the presence of three highly conserved regions (10-15 amino acids each), spatially well-separated in the sequence, that constitute the active site architecture. Indeed, the residues in the conserved regions of three monomers build up each active site, since the homotetramer contains four identical active sites that are located at the interface of three different subunits each. ${ }^{4}$ A portion of the third conserved region is a flexible loop, named the SS-loop, that 
is crucial for the catalytic mechanism since it carries an essential catalytic base and has been shown to close over the rest of the active site after substrate binding. ${ }^{84}$
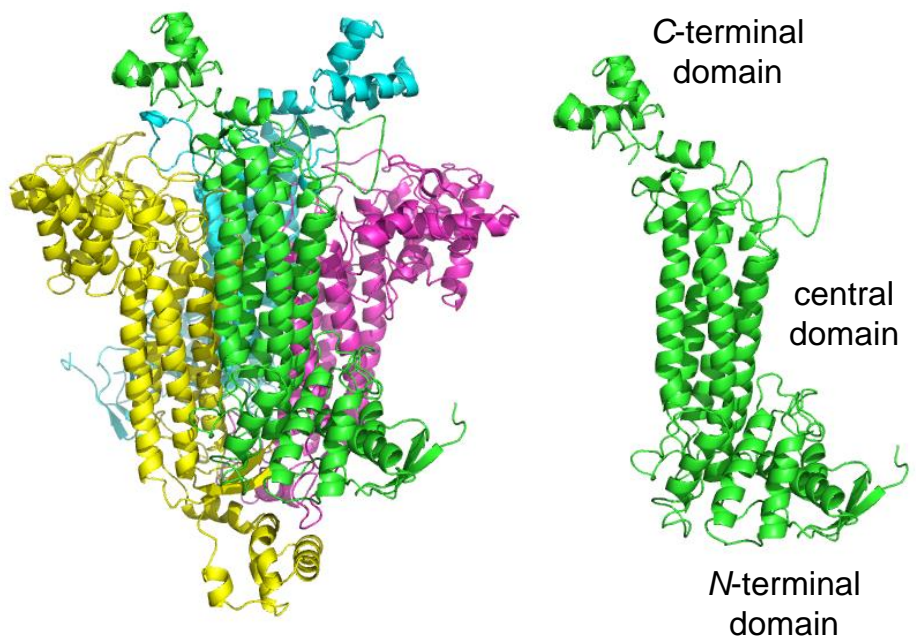

Figure 1. X-ray crystal structure of EcDAL (PDB ID: 1JSW), showing the homotetramer and the domains of each subunit.

AspA and other DALs were originally shown to be allosterically activated by a divalent metal ion and by L-aspartate, ${ }^{85}$ but other enzymes of the family do not display these features. The location of the binding site for these activators, independent from the catalytic site, has not been identified in the crystal structure of AspA.

A general acid-base mechanism has been proposed for aspartases and related enzymes (Scheme 4), with strong evidence from $\mathrm{pH}$-rate profiles and inhibition studies for DALs, FUMs, ADLs and ARLs. ${ }^{4,86}$ In the first step, after substrate binding and loop closure, the pro- $R$ proton at the $\beta$ position is abstracted by the essential catalytic base on the flexible loop, forming a carbanion stabilized as its aci-carboxylate (ene-diolate) form. This intermediate then rearranges with concomitant cleavage of the $\mathrm{C}-\mathrm{N}$ bond ( $\mathrm{C}-\mathrm{O}$ bond for fumarases), leading to the release of the nucleophile (ammonia, amine or water) and fumarate. Such cleavage is the rate determining step, 
which may be facilitated by the presence of a general acid to donate a proton to the leaving group. Since the elimination is anti, two separate residues must be involved in proton abstraction and proton donation. For most of the superfamily members the general acid is catalytically essential, but for aspartases it has not yet been possible to establish if the leaving group is released as an ammonium ion or as free ammonia. The latter case might make the presence of a general acid to protonate the $\alpha$-amino group unnecessary, and indeed a catalytically essential acid residue in the active site has not yet been identified by mutagenesis studies.

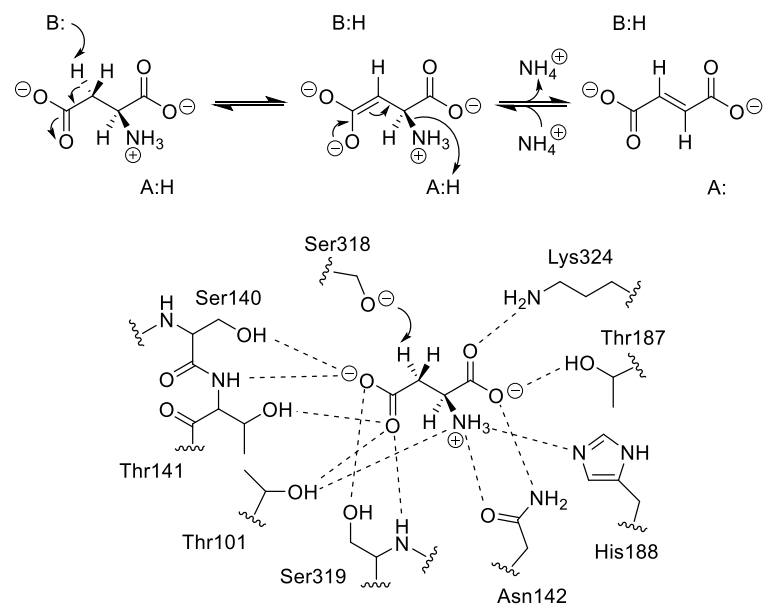

Scheme 4. Catalytic mechanism of DALs and schematic representation of the active site of AspB.

This general acid-base mechanism is also supported by inhibition experiments carried out with substrate analogues bearing a nitro group instead of the $\beta$-carboxylate (Scheme 5). The aspartase from Hafnia alveis was shown to be inhibited competitively by such compounds, binding them several hundred times more tightly than its natural substrates. ${ }^{87}$ These results suggest that the anions behave like transition state analogues and that the mechanisms of the aspartase reaction involves an enzyme-bound 3-carbanion. Additional supporting evidence comes from an X-ray crystal structure of AspB with L-aspartate bound. ${ }^{84}$ This structure shows the substrate in a distorted conformation similar to that of the ene-diolate intermediate, stabilized by an extensive 
network of hydrogen bonds. This might allude to a facilitated proton abstraction from the $\mathrm{sp}^{3}$ carbon caused by binding-induced distortion of the geometry of the substrate, forcing it to adopt a conformation similar to the $\mathrm{sp}^{2}$ ene-diolate intermediate.

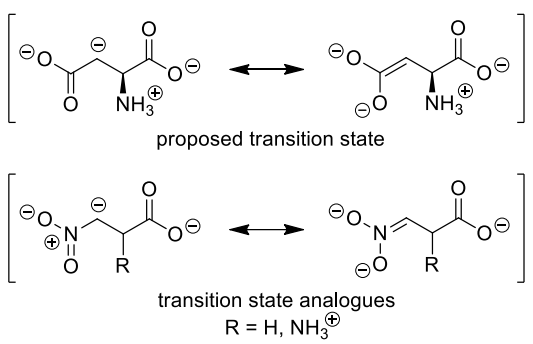

Scheme 5. Nitro analogues of the ene-diolate intermediate.

The structure of AspB with L-aspartate bound also showed for the first time the closedconformation of the enzyme (similar to what was previously observed for other superfamily members, e.g. ADL), allowing the identification of Ser318 as the essential catalytic base. This was also confirmed by mutagenesis, since no activity was detected with the Ser318Ala mutant. ${ }^{84}$ However, to function as a base, the serine residue must be deprotonated to the corresponding oxyanion, and the mechanism by which this serine anion is formed and stabilized has not yet been elucidated.

Concerning substrate specificity, aspartases are among the most specific enzymes known, with extensive studies failing to identify any alternative amino acid substrate. Many early reports already proved lack of activity with D-aspartate, other proteinogenic L-amino acids, other unsaturated acids (e.g., crotonate, maleate, mesaconate, aconitate) and related compounds. ${ }^{74,88,89}$ Remarkably $\mathrm{Ma}$ et al. reported an alteration of specificity from L-aspartate to L-asparagine by prolonged incubation with different concentrations of ethanol, although this work was never exploited for preparative applications. ${ }^{90}$ The case of L-aspartic- $\beta$-semialdehyde is worth noting: even though this compound is a non-natural substrate that is deaminated by the enzyme, it leads 
to irreversible inactivation by chemical reaction with active site residues, i.e., it behaves as a suicide substrate. ${ }^{91}$ Additional experiments showed that after deamination and further reaction with the active site, the aldehyde group is still intact, and that the amino acid residue that is covalently modified is a cysteine. Subsequently, Falzone et al. performed a very extensive screening of structural analogues of aspartate, in the attempt to find alternative substrates. While this was not successful, it led to the identification of several inhibitors with various $K_{i}$ values, ranging from 0.2 to $36 \mathrm{mM}$ (Scheme 6 ) ${ }^{85}$ The binding seems to strictly require one polar group at each end of the short carbon chain, while greater variation is tolerated at the $\alpha$-position. Nevertheless, in spite of the high specificity of aspartases, a few interesting examples of promiscuity and successful engineering have been reported, as described in Section 4.1.

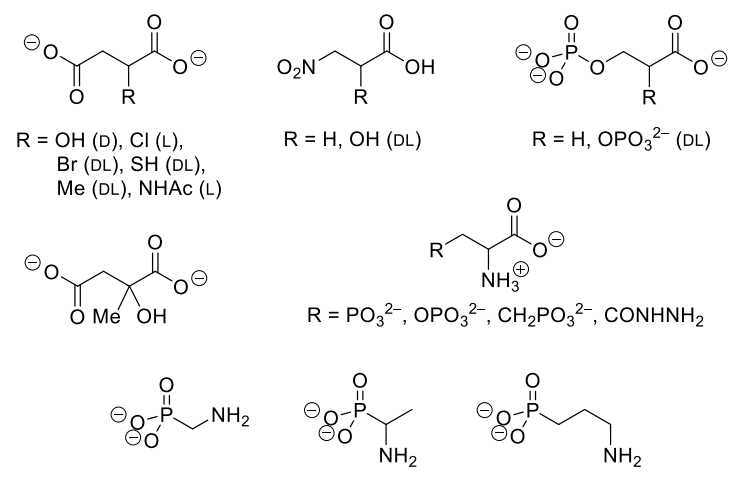

Scheme 6. Inhibitors of AspA identified in the screening for potential non-natural substrates.

\subsection{Methylaspartate ammonia-lyases (MALs)}

While investigating the reversible conversion of glutamate to mesaconate and ammonia by Clostridium tetanomorphum, Barker et al. discovered that by treating the cell-free extract with charcoal some essential factors were removed, resulting in no activity when starting from glutamate, and accumulation of a different amino acid when starting from mesaconate and ammonia. The amino acid was found to be L-threo-3-methylaspartate, and this led to the isolation of the first methylaspartase (CtMAL) in $1958 .^{92,93}$ Facultative anaerobes such as Proteus sp., 
Enterobacter sp., Morganella morganii and Citrobacter sp. have also been shown to harbor MAL activity, as evidenced through cell-free assays ${ }^{94}$ and/or protein isolation. ${ }^{95}$ Intriguingly, it was also observed that in the reverse reaction, the enzyme catalyzes not only anti-addition, but also synaddition of ammonia, forming L-erythro-3-methylaspartate as a minor product (Scheme 7). ${ }^{96}$

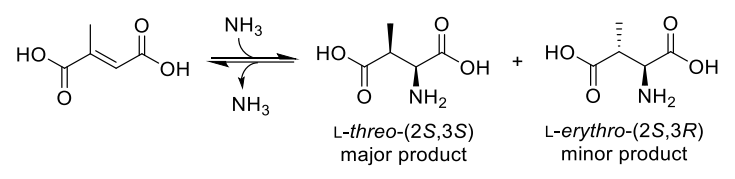

Scheme 7. Reaction catalyzed by MALs.

CtMAL was first produced recombinantly in $E$. coli in $1992,{ }^{97}$ followed by other methylaspartases from different sources, including Citrobacter amalonaticus (CaMAL), ${ }^{98}$ Fusobacterium varium (FvMAL) ${ }^{99}$ and Carboxydothermus hydrogenoformans (ChMAL). ${ }^{100}$ The latter is of particular interest due to its thermostability and higher activity at high temperatures. MALs are homodimeric proteins that require divalent $\left(\mathrm{Mg}^{2+}\right)$ and monovalent $\left(\mathrm{K}^{+}\right)$cations for activity. The catalytic mechanism has been debated for many decades, with various hypotheses proposed, including a carbanion mechanism similar to that of DALs (Section 2.1) or the involvement of an active site modification similar to that of PALs (Section 3.1). However, the Xray crystal structure analysis of ligand-free $\mathrm{CtMAL}^{6}$ and of the complex of CaMAL with L-threo3-methylaspartate, ${ }^{5}$ both published in 2002, solved the conflict. The MAL subunit is composed of a large $C$-terminal domain that has the structure of an 8 -fold $\alpha / \beta$-barrel (TIM barrel), and a smaller $N$-terminal domain, composed mainly of $\beta$-strands, that wraps around the barrel and partially occludes its $C$-terminus (Figure 2). 

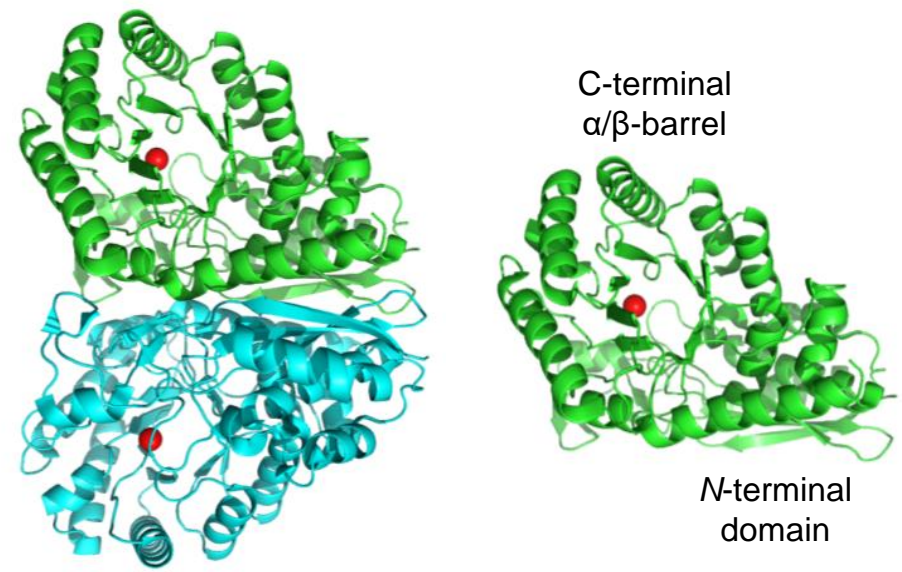

Figure 2. X-ray crystal structure of CtMAL (PDB ID: 1KCZ), showing the homodimer and the domains of each subunit. The $\mathrm{Mg}^{2+}$ ions are shown in red.

Therefore, from a structural point of view, MALs were found to be totally unrelated to either DALs or MIO-enzymes, and instead to belong to the enolase superfamily, despite the very low sequence similarity (clearly illustrating the tolerance of such fold for amino acid substitutions). The members of this family catalyze a broad range of transformations, including $\beta$-eliminations of water, racemizations and cycloisomerizations, all of which share a common metal-assisted general base catalyzed abstraction of a proton from the $\alpha$-position of a carboxylic acid, generating a stable enolate intermediate. ${ }^{7,101}$ The structures of CtMAL and CaMAL, combined with sequence alignments with other members of the superfamily, enabled the identification of the magnesium ion binding site and the catalytic residue responsible for the proton abstraction (Lys331). This suggested a mechanism based on the removal of the $\beta$-proton of 3-methylaspartate mediated by the catalytic lysine, formation of the stable ene-diolate (further stabilized by the magnesium ion) and collapse of the intermediate to release ammonia (Scheme 8). ${ }^{102}$ 

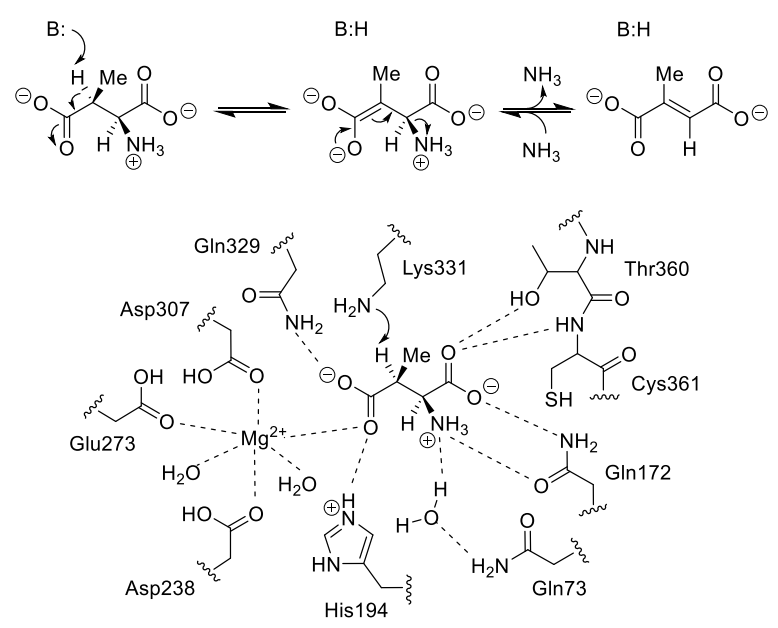

Scheme 8. Catalytic mechanism of MALs and schematic representation of the active site of CtMAL.

The magnesium ion sits on top of the $C$-terminal mouth of the $\alpha / \beta$-barrel, tightly bound by three carboxylic acid residues (Asp238, Glu273 and Asp307). Three molecules of water complete the octahedral coordination. Upon substrate binding, one of these water molecules is replaced by the oxygen atoms of the $\beta$-carboxylate group of the substrate, stabilizing the enolate intermediate, and effectively lowering the $\mathrm{p} K_{a}$ of the $\alpha$-proton. The residue of Lys331 was identified as the base responsible for the proton removal from the L-threo substrate, and it was postulated that His194 (superimposable with key basic residues of other members of the enolase superfamily) could act as the base for the removal of the proton of the L-erythro substrate. Indeed, it has been demonstrated with other enolase enzymes (e.g., mandelate racemase, glucarate dehydratase) that the enolate intermediate can be converted into different stereoisomeric products by different residues suitably placed in the active site. ${ }^{6}$

These considerations, and this mechanism of MAL, are strongly supported by mutagenesis studies. ${ }^{103,104}$ Poelarends and co-workers demonstrated that MAL does not catalyze the direct epimerization of the L-threo and L-erythro products, then investigated the effect of several mutations of the relevant active site residues. The Lys331 residue was confirmed to be essential 
for activity, since variants harboring mutations to Ala, Gly, His, Gln or Arg completely lost MAL activity. On the other hand, the His194Ala mutation results in perfect diastereoselectivity for the L-threo product, albeit with considerably reduced activity due to the loss of some stabilizing hydrogen bond interactions. ${ }^{103}$ A similar outcome was observed, to a lesser extent, with the Gln329Ala mutation. ${ }^{104}$ Several other residues lining the active site were subjected to sitedirected mutagenesis and some were found to be critical for substrate binding and activation (particularly Gln73, Gln172, Tyr356, Thr360 and Leu384), with obvious benefits for future engineering studies. ${ }^{104}$

With this combination of structural information, biochemical studies and mutagenesis experiments, the mechanism of MAL catalyzed reactions has been elucidated, leading to further work on expanding the substrate scope of these enzymes by engineering and evolution, which will be described in Section 4.1 .

\section{Structure and mechanism of aromatic amino acid ammonia-lyases and aminomutases}

\subsection{Arylalanine ammonia-lyases (PALs, HALs and TALs)}

The non-oxidative deaminations of L-phenylalanine, L-tyrosine and L-histidine (mediated by PALs, TALs and HALs, respectively) to form the corresponding $\alpha, \beta$-unsaturated acids (cinnamic acid, $p$-coumaric acid and urocanic acid) have different metabolic and catabolic functions in various organisms.

HALs play a major role in amino acid degradation. While catabolic pathways of most amino acids usually start with the oxidative removal of the $\alpha$-amino group by transaminases, in the case of histidine this process begins with the HAL mediated non-oxidative deamination, yielding urocanate that is further converted to $\mathrm{N}$-formiminoglutamate. The latter is either used for the synthesis of 5-formiminotetrahydrofolate (in mammals), or is further degraded to glutamate (in 
bacteria and some fungi). Interestingly, the groups of eukaryotes found not to employ HALs in primary metabolism (Dikarya and land plants) are composed of organisms known to encode polypeptides with PAL activity, acquired through horizontal gene transfer. ${ }^{105}$

PALs, on the other hand, are needed for biosynthetic purposes. The conversion of phenylalanine into cinnamic acid is the first step of phenylpropanoid biosynthesis in plants, which affords a stunningly broad collection of polyhydroxylated aromatic compounds (e.g., lignans, coumarins, flavonoids, stilbenes, anthocyanins). ${ }^{106}$ By siphoning the primary metabolite phenylalanine into polyphenol synthetic pathways, PAL effectively acts as a gateway enzyme between primary and secondary metabolism. TALs serve the same role (mainly as an alternative pathway to $p$ coumaroyl-CoA), but they are involved in more specific pathways, possibly adding evidence to the speculation that they are rarer than PALs. ${ }^{107}$ However, considering that the $p$-coumaric acid product of the TAL reaction can also be derived from the cinnamic acid product of the PAL reaction by means of cinnamic acid 4-hydroxylase $(\mathrm{CAH})$, the metabolic functions of PALs and TALs are strongly related.

Arylalanine ammonia-lyases, generally known as class I lyase-like enzymes, together with the structurally related aminomutases (see Section 3.2), are fairly ubiquitous in nature, with PALs and TALs being less common in bacteria. A large number of those have been characterized, produced recombinantly in E. coli and purified to homogeneity.

The most studied enzymes of this family are RgPAL from Rhodotorula glutinis, ${ }^{108}$ RtPAL from Rhodosporidium toruloides (formerly known as Rhodotorula gracilis), ${ }^{109}$ PcPAL from Petroselinum crispum (parsley), ${ }^{110,111}$ AvPAL from Anabaena variabilis, ${ }^{112}$ RsTAL from Rhodobacter sphaeroides, SxTAL (BagA) from Streptomyces sp., ${ }^{113}$ PfHAL from Pseudomonas fluorescens, ${ }^{114}$ and PpHAL from Pseudomonas putida. ${ }^{115}$

Obviously, the reactions catalyzed by arylalanine ammonia-lyases are chemically more challenging than those of DALs and MALs, since the abstraction of a proton from the $\beta$-carbon is unfavorable due to the very low acidity ( $\mathrm{p} K_{a} \sim 40$ ) of the $\beta$-protons. Indeed, it is quite surprising 
that these enzymes do not require complex cofactors and radical mechanisms as seen, for instance, with some aminomutases. This has posed a considerable mechanistic challenge to biochemists and, after half a century of research, the answer is still not completely clear.

The very first mechanistic studies performed on HAL identified a catalytically essential electrophile, derived either from a tightly bound cofactor or from active site functionalization. ${ }^{116}$ Inactivation by the radioactively labelled nucleophiles $\left[{ }^{3} \mathrm{H}\right]$-borohydride and $\left[{ }^{14} \mathrm{C}\right]$-cyanide afforded, upon complete hydrolysis, $\left[{ }^{3} \mathrm{H}\right]$-alanine and $\left[{ }^{14} \mathrm{C}\right]$-aspartate, respectively, suggesting that the electrophilic group was dehydroalanine (acting as a Michael acceptor). ${ }^{108,115,117,118}$ It was also recognized that the nitrogen of dehydroalanine formed a Schiff base with a carbonyl compound that could not be identified. ${ }^{117}$ Site-directed mutagenesis studies in PpHAL, ${ }^{119}$ PcPAL $^{120}$ and RsTAL ${ }^{121}$ demonstrated that an active site serine residue is the precursor of the catalytically essential dehydroalanine (Ser143 in PpHAL, Ser102 in PcPAL, Ser150 in RsTAL). Interestingly, the HAL and PAL mutants where that serine was replaced with a cysteine showed identical catalytic properties, suggesting that the dehydroalanine-derived functionality could be formed by post-translational modification via elimination of either $\mathrm{H}_{2} \mathrm{O}$ or $\mathrm{H}_{2} \mathrm{~S} .{ }^{122,123}$

Unexpectedly, with the first X-ray crystallographic study of PpHAL, Schulz and co-workers showed that the electron density around the predicted dehydroalanine residue was not consistent with any of the standard proteinogenic amino acids, and the electrophilic functionality in the active site was more complex than expected. The observed structure was the five-membered heterocycle 4-methylideneimidazol-5-one (MIO, formally 5-methylene-3,5-dihydro-4H-imidazol4-one). ${ }^{124}$ The MIO moiety, very often inappropriately called "MIO cofactor" or "MIO prosthetic group" (as briefly discussed in Section 1.1.4), is in fact a dehydroalanine derivative formed by cyclization and double dehydration of an active site tripeptide, typically Ala-Ser-Gly and, less frequently, Thr/Ser/Cys-Ser-Gly (Scheme 9). ${ }^{14,17,71}$ 


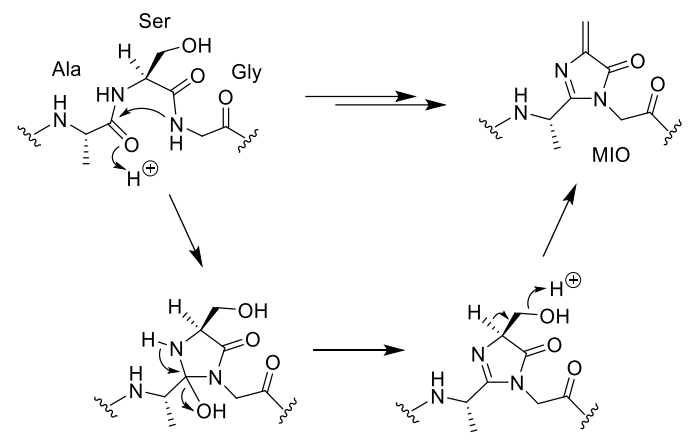

Scheme 9. Mechanism of formation of the MIO electrophile by cyclization and double dehydration of the active site tripeptide Ala-Ser-Gly.

The mechanism of formation of the MIO electrophile is post-translational and autocatalytic, since heterologous expression of prokaryotic and eukaryotic PALs and HALs results in fully active enzymes. It closely resembles the formation of the fluorophore of green fluorescent protein (GFP), discovered in $1996,{ }^{125}$ but, in contrast with arylalanine ammonia-lyases, the cyclizing tripeptide in GFP is Ser/Thr-Tyr-Gly, and the final step is an oxidation by molecular oxygen instead of the second elimination of water. ${ }^{126}$ A very recent molecular dynamics simulation study provided great insight into the process of MIO formation in PpHAL, supporting the idea that mechanical compression from neighboring residues is required to prevent the formation of stabilizing hydrogen bonds and to enforce the correct alignment of donor and acceptor orbitals. ${ }^{127}$ The crystal structure of PpHAL also showed for the first time the general structural features of arylalanine ammonia-lyases, homotetramers mainly built of $\alpha$-helices of varying lengths ${ }^{128}$. Each subunit contains a globular $N$-terminal domain and an elongated $C$-terminal domain with five parallel $\alpha$-helices, surrounded by six more (Figure 3). The tetrameric structure contains four identical active sites, each built of residues belonging to three different subunits. ${ }^{124}$ Certain plant and fungal PALs have been shown to bear an additional $C$-terminal multi-helix domain, which may play a role in the fine regulation of enzyme activity in these organisms via substrate channeling and/or overall destabilization of the protein. ${ }^{110,129}$ Structural alignments of these 
domains point to their origins as partial duplication or gene fusion events within the evolutionary history of plant and fungal PALs. ${ }^{130}$ Other than the presence of this additional domain, the general structural features of arylalanine ammonia lyases are found invariably in all the known crystal structures of the members of this family, e.g., RtPAL, ${ }^{109}$ PcPAL $^{110}$ and AvPAL. ${ }^{112}$ All of the enzymes that have been structurally characterized were found to contain the MIO moiety (already demonstrated by spectroscopic methods ${ }^{131}$ ) and to have almost identical active site geometry (Scheme 10a).
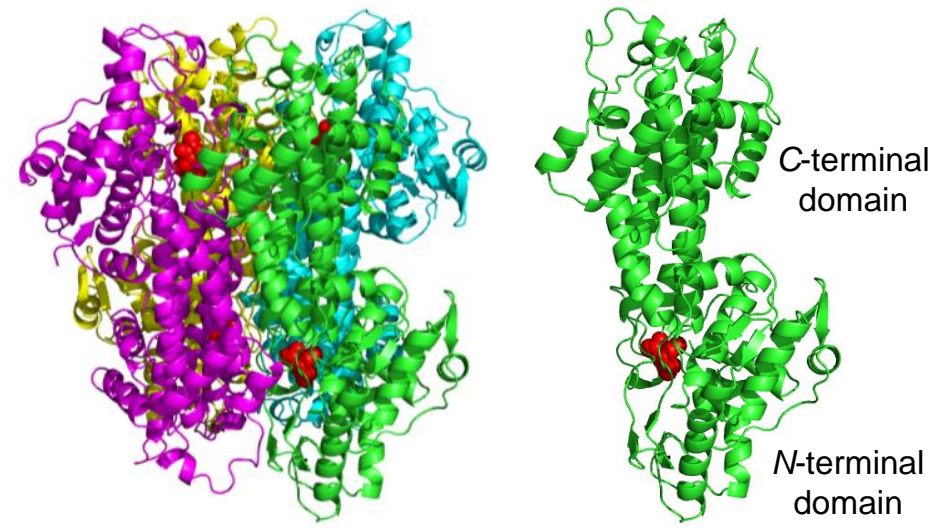

Figure 3. X-ray crystal structure of PpHAL (PDB ID: 1B8F), showing the homotetramer and the domains of each subunit. The MIO heterocycles are shown in red. 

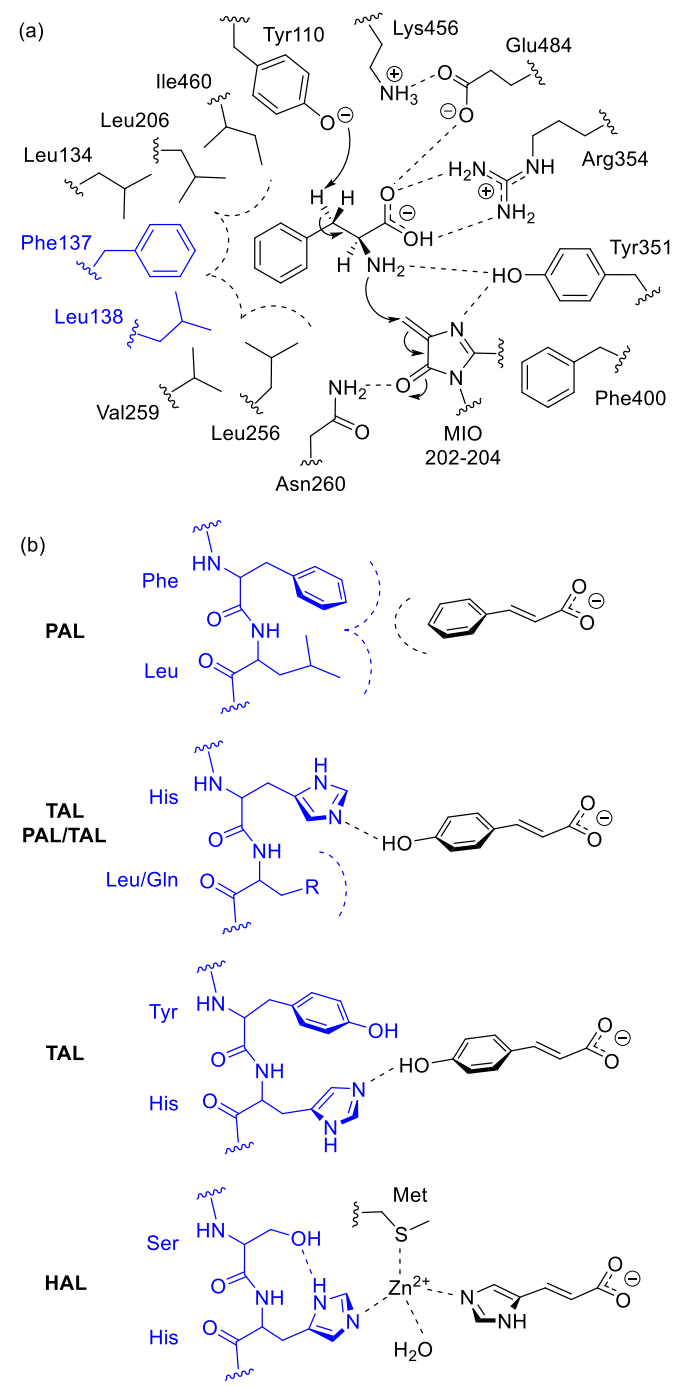

Scheme 10. Schematic representation of the active site of PcPAL (a) and putative roles of the socalled selectivity residues in substrate recognition (b). Selectivity residues are shown in blue.

Just a few years later, the first crystal structure of a TAL was reported (RsTAL from Rhodobacter sphaeroides), ${ }^{132}$ demonstrating that only one residue (His89 in RsTAL) is responsible for its selectivity for tyrosine. The single point mutation His89Phe resulted in a complete selectivity switch from TAL to PAL activity, as was also demonstrated independently and simultaneously by Schmidt-Dannert and co-workers. ${ }^{107}$ Analogously, the Phe144His mutation introduced in AtPAL1 from Arabidopsis thaliana gave the opposite switch from PAL to TAL activity. ${ }^{107}$ These 
remarkable examples of modulation of substrate specificity are rather rare; an earlier attempt to turn PcPAL into a HAL by replacing the only two non-conserved active site residues (Leu138His-Gln488Glu) gave a mutant much less active on phenylalanine and with a $K_{M}$ for histidine almost identical to that of HAL, albeit with much lower $k_{\text {cat }}{ }^{133}$ These results indicate the subtle influence residues far from the active site can have, and also reinforce the idea of the active site glutamate at the second position being a determining feature of HALs (as glutamine is found in all other family members). ${ }^{133,134}$ More recently, examples of enzymes with TAL activity (e.g., SxTAL/BagA from Streptomyces) ${ }^{113}$ or dual phenylalanine-tyrosine specificity (e.g., ZmPAL1 from Zea mays) ${ }^{135}$ have been identified with the non-homologous histidine active site residues being seemingly responsible for TAL activity, presumably due to the interaction of these two residues with the para-substituent of the substrate. The existence of these enzymes, combined with the known bifunctionality of some fungal enzymes point to multiple convergent evolution events resulting in TAL activity within different clades of the class I lyase-like enzyme family. As variation at either position from hydrophobic residues to histidine is shown to confer tyrosine or histidine acceptance, these two active site positions act as "selectivity residues" (Scheme 10b). In particular, for PAL activity hydrophobic interactions are predominant, for TAL activity the histidine nitrogen interacts with the para-hydroxy functionality of the substrate, and for HAL activity (recognized in early studies to depend on $\mathrm{Zn}^{2+}$ ions ${ }^{136}$ ) the histidine residue and the histidine substrate are proposed to coordinate the divalent cation, as shown by detailed computational simulations. ${ }^{137}$ Examples of the most typical selectivity residue combinations seen in the aromatic binding pocket, and the corresponding substrate specificities, are shown in Table 3. 
Table 3. Examples of ammonia-lyase substrate specificities and selectivity residues.

\begin{tabular}{lll}
\hline Activity & Selectivity residues & Examples \\
\hline PAL & Phe-Leu & PcPAL, AvPAL, AtPAL1 \\
TAL & Tyr-His & BagA \\
TAL & His-Leu & RsTAL \\
PAL/TAL & His-Leu & ZmPAL1 \\
PAL/TAL & His-Gln & RgPAL, RtPAL \\
HAL & Ser-His & PpHAL, PfHAL \\
\hline
\end{tabular}

Mutagenesis studies on PpHAL, ${ }^{138} \mathrm{PcPAL}^{133}$ and $\mathrm{RsTAL}^{121}$ revealed also the presence of an essential catalytic tyrosine residue (Tyr53 in PpHAL, Tyr110 in PcPAL, Tyr60 in RsTAL) on a flexible loop covering the binding site. In contrast with PpHAL, the first crystal structure of a PAL did not show a complete picture of the active site, since the flexible loop carrying the catalytic tyrosine was either absent (RtPAL) or locked in the inactive open conformation (PcPAL), as shown in Figure 4. By homology modelling, using the closed-loop structure of PpHAL as a guide, a closed loop structure of PcPAL was built, along with those of two bacterial homologs. ${ }^{129}$ Dynamic simulations led to the hypothesis that the additional $C$-terminal domain of eukaryotic PALs (absent in bacterial PALs) destabilizes the enzyme, thus decreasing its lifetime, a potentially important feature in the regulation of phenylpropanoid biosynthetic pathways. Further focused directed evolution work around the carboxylate/amine binding sites only led to variants with decreased activity, highlighting the presence of a complex and highly mutationsensitive network of hydrogen bonds. ${ }^{139}$ 


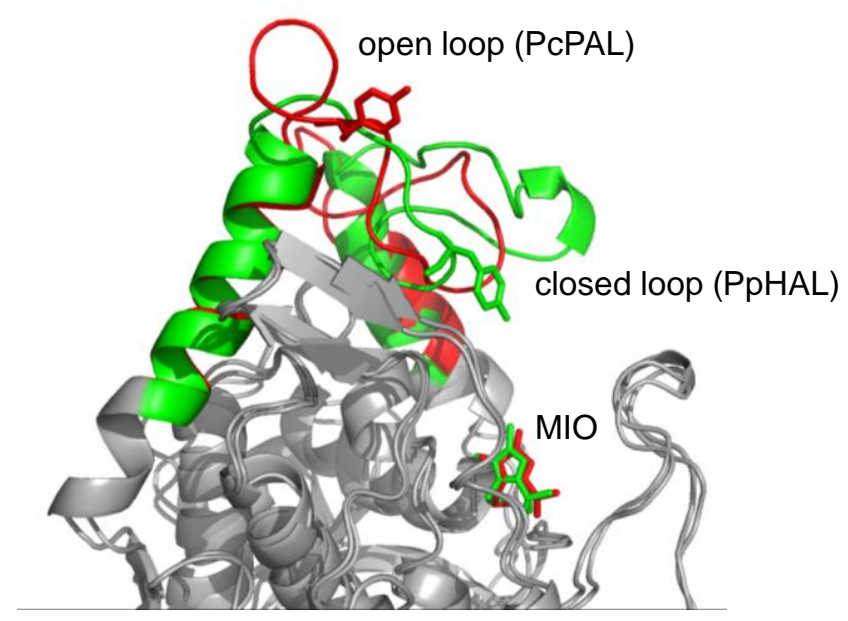

Figure 4. Overlay of the X-ray crystal structures of PpHAL (PDB ID: 1B8F) and PcPAL (PDB ID: 1W27). The loop bearing the catalytic tyrosine is locked in the closed conformation in the structure of PpHAL (green), but in an inactive open conformation in the structure of PcPAL.

It is worth mentioning that the crystallization of PpHAL required the mutagenesis of a surface cysteine to alanine (Cys273Ala) in order to obtain high quality crystals reproducibly. ${ }^{124}$ A similar strategy, applied to the recently discovered and characterized AvPAL from Anabaena variabilis, ${ }^{112}$ allowed researchers to obtain the first crystal structure of a PAL with all the residues in the catalytically active closed conformation. In this case, mutation of two surface cysteine residues was required (AvPAL-Cys503Ser-Cys565Ser) and the solution of the structure afforded the first complete picture of the active site of a PAL. ${ }^{130}$

The mechanism of MIO-dependent ammonia-lyases has been debated for many decades, and is still considered partly controversial. The most likely mechanistic paths proposed are summarized in Scheme 11. 


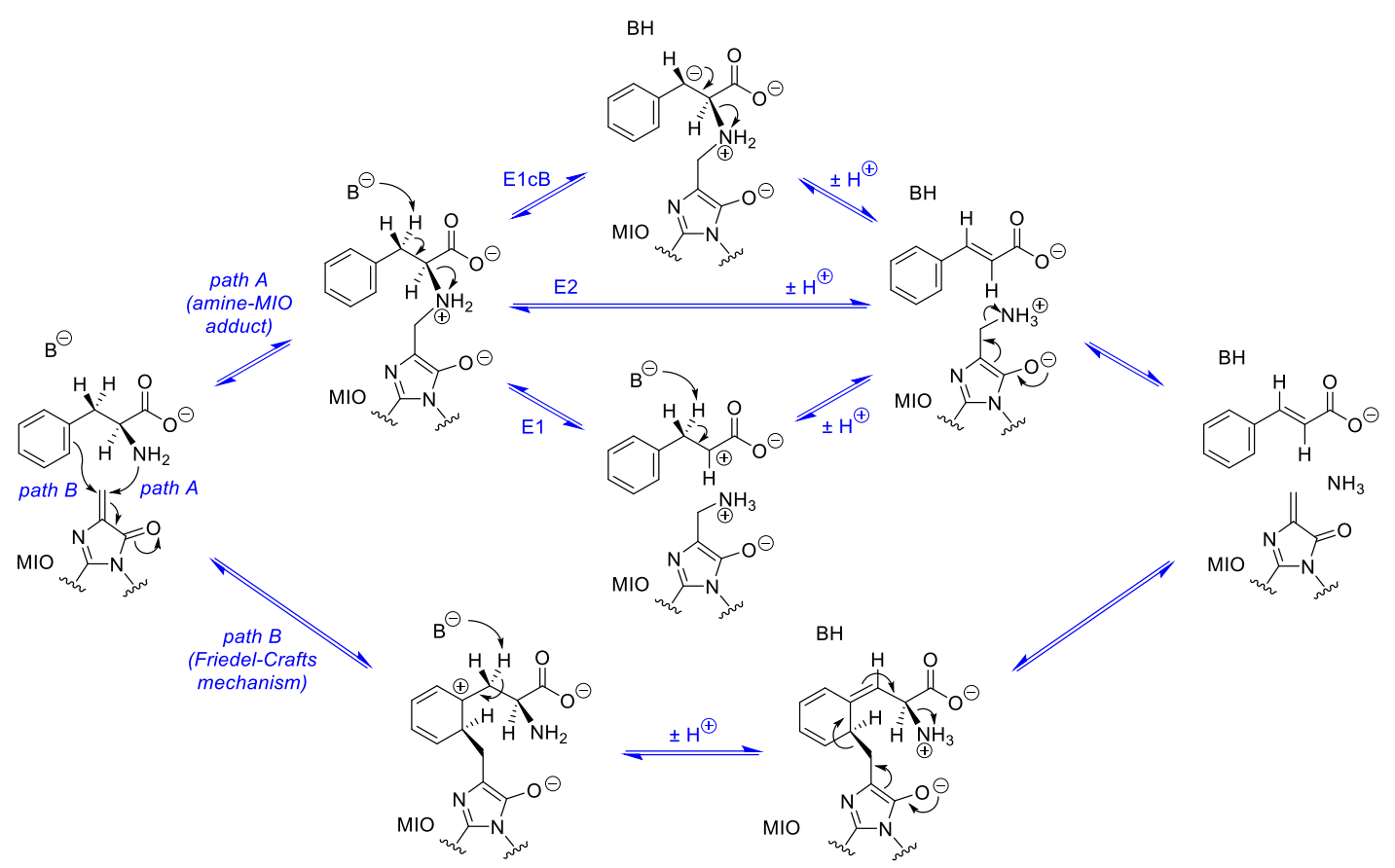

Scheme 11. Mechanisms proposed for the deaminations mediated by arylalanine ammonia-lyases.

The first proposal by Hanson and Havir, ${ }^{118}$ later revised by Cleland and co-workers, ${ }^{140}$ was formulated even before the structure of the MIO had been fully elucidated. This reaction mechanism (Scheme 11, path A) involved a direct interaction of the electrophilic functionality with the deprotonated amino group of the substrate, forming a covalent amino-MIO intermediate. This step facilitates the reaction by forming a secondary ammonium ion, a better leaving group than less substituted species. Then, the catalytically essential enzymatic base abstracts the pro- $S$ proton from the $\beta$-position, forming a carbanion intermediate, immediately followed by elimination of the MIO- $\mathrm{NH}_{2}$ leaving group (E1cB mechanism). Lastly, reprotonation of the MIO$\mathrm{NH}_{2}$ moiety and elimination of ammonia completes the cycle. The $\beta$-proton abstraction and the MIO- $\mathrm{NH}_{2}$ elimination can, in principle, occur according to three different pathways: E1 (via a carbocation intermediate), E2 (concerted) and E1cB (via a carbanion intermediate), as shown in Scheme 11. However, the E1 mechanism is extremely unlikely, not only due to the high energy of the $\alpha$-carbocation intermediate, but also because of the incompatibility with the orientation of 
helix dipoles, as observed, for instance, in RtPAL. ${ }^{109}$ On the basis of the kinetic isotope effects observed with ${ }^{15} \mathrm{~N}$-labelled and $\beta-{ }^{2} \mathrm{H}_{2}$-labelled phenylalanines, the concerted E2 mechanism was also originally excluded. ${ }^{140}$ However, this statement is strictly valid only if the elimination step is rate-limiting, and a detailed computational study on the mechanism of RsTAL reactions indicated that this is not necessarily the case. ${ }^{141}$ In this work, Poppe and co-workers revealed a tandem nucleophilic and electrophilic enhancement by a proton transfer from the protonated amino group of the zwitterionic substrate (increasing the nucleophilicity of the nitrogen) to the nitrogen of the MIO ring (increasing the electrophilicity of the double bond). This step is mediated by Tyr300, a residue found to be fully conserved not only in all known 3D structures of MIO-enzymes, but also across the gene sequences (e.g., Tyr351 in PcPAL, Scheme 10a). The calculations suggested that such proton transfer is the rate-determining step of the reaction, possibly justifying why no significant ${ }^{2} \mathrm{H}$ or ${ }^{15} \mathrm{~N}$ isotope effects could be observed experimentally.

In 1995, a completely different mechanistic pathway was proposed by Rétey and coworkers. ${ }^{142,143}$ This mechanism (Scheme 11, path B) involves first a Friedel-Crafts-like attack of the MIO electrophile with formation of a covalent ring-MIO intermediate, which renders the $\beta$ proton more acidic by generating a positively charged complex. The Friedel-Crafts-like complex then decomposes with elimination of an ammonium ion, to restore the aromaticity of the system and regenerate the MIO. ${ }^{144,145}$ This mechanism is plausible for electron-rich aromatic rings such as histidine or tyrosine, but less plausible for the PAL reaction on unsubstituted substrates. Evidence to support this mechanism includes the relative rates of different substrates due to resonance effects (e.g., much higher reaction rates of $m$-tyrosine with PAL compared to phenylalanine), ${ }^{143}$ and the stereoelectronic viability observed in the reaction of a suitablydesigned synthetic model compound. ${ }^{146}$

A very recent computational study also highlighted the possibility of a single-step mechanism for the TAL reaction, without the formation of covalent bonds with the MIO. ${ }^{147}$ This hypothesis, however, was considered unlikely on the basis of serious contradictions with experimental data, 
such as the kinetic isotope effects, ${ }^{140}$ the stepwise release of the arylacrylate prior to that of ammonia, ${ }^{148}$ and the reversibility even at relatively low ammonia concentrations. ${ }^{149}$

The amino-MIO mechanism is now generally accepted as the most plausible, primarily on the basis of structural data (e.g., RsTAL with the inhibitor 2-aminoindan-2-phosphonate bound, ${ }^{150}$ and various related aminomutases, see Section 3.2), computational simulations ${ }^{141}$ and evidence of conversion of substrates that cannot react via a Friedel-Crafts-like mechanism (e.g., pentafluorophenylalanine ${ }^{151}$ or propargylglycine ${ }^{149}$ ). The main problem of the difficulty of abstraction of a non-acidic $\beta$-proton in the $\mathrm{E} 1 \mathrm{cB}$ mechanism, has not found a convincing explanation as of yet (although examples of abstraction of an apparently non-acidic proton vicinal to a good leaving group performed by hydroxide ions are known). On the other hand, the E2 mechanism overcomes this difficulty, and computational data also showed that substrate binding to form the amino-MIO intermediate caused a weakening of the $\mathrm{C}_{\alpha}-\mathrm{N}$ bond and, at the same time, increased the acidity of the $\beta$-proton, potentially enabling a smooth E2 elimination. ${ }^{141}$ The debate between the $\mathrm{E} 1 \mathrm{cB}$ and the $\mathrm{E} 2$ mechanisms will probably continue until conclusive experimental evidence is found.

The conversion of arylalanines bearing a strongly electron-withdrawing group (EWG) is also worth mentioning because of its mechanistic implications. The conversion of 4-nitrohistidine with HAL was reported by Klee et al. in 1979. ${ }^{152}$ In addition, no deuterium kinetic isotope effect was observed with $\beta-{ }^{2} \mathrm{H}_{2}-4$-nitrohistidine, as opposed to unsubstituted $\beta-{ }^{2} \mathrm{H}_{2}$-histidine. This can be explained by considering that the decrease in electron-density of the ring leads to an increased acidity of the $\beta$-protons, thus making the abstraction of the pro- $S$ hydrogen no longer the rate limiting step. More recently, Rétey and co-workers demonstrated the conversion of nitrophenylalanine with PcPAL, in both the deamination and the amination directions, showing also that the MIO-less mutant PcPAL-Ser202Ala is active on such substrates, even with considerably higher specific activities than the natural substrates. ${ }^{153}$ This was thought to be further evidence of a Friedel-Crafts-like mechanism, since the strong electron-withdrawing effect 
of the nitro group provides the same activation as that provided by the MIO moiety after the Friedel-Crafts-type attack. Very recently, however, we showed evidence of a MIO-independent competing reaction pathway with highly activated substrates (i.e., those carrying strong EWGs), that proceeds non-stereoselectively and more slowly. ${ }^{154}$ With isotopic labelling and mutagenesis studies it was shown that this pathway is consistent with an E1cB mechanism and proceeds via stereoselective deprotonation of the pro- $R \beta$-proton of D-phenylalanine, followed by either nonselective reprotonation of the carbanion, or elimination of ammonia. This remarkable MIOindependent activity could be increased by engineering ${ }^{155}$ (and exploited in biocatalytic applications, see Section 4.3), but attempts to engineer a truly D-selective PAL are currently unsuccessful.

In spite of the ongoing mechanistic debate, which will certainly promote more discussion and research in the future, aromatic amino acid ammonia-lyases (PALs in particular) have been employed very effectively in multiple practical applications, ranging from the synthesis of amino acids, to assays and biosensors, to the development of new therapeutic strategies. These applications will be discussed in Sections 4 and 5.

\subsection{Arylalanine aminomutases (PAMs and TAMs)}

The first example of a MIO-dependent 2,3-amine shift was reported in 2003 through the study of enediyne antitumor natural products in Streptomyces globisporus. ${ }^{156}$ The chromophore of the C0127 secondary metabolite under study was known to contain an unusual 3-hydroxy-5-chloro- $\beta$ tyrosine of $(S)$-configuration (Scheme 12a), despite the absence of any open reading frames predicted to have traditional aminomutase chemistry in the cloned biosynthetic gene cluster. Investigation of the gene encoding SgcC4 revealed it shared homology with known HAL and PAL enzymes, as well as its $(S)$-selective tyrosine aminomutase (or $(S)$-TAM) activity. The association of MIO-dependent catalysis was also confirmed via borohydride and cyanide inactivation, as well as mutagenesis of the central residue essential for catalytic moiety 
formation. ${ }^{156}$ Further evidence was added to this assertion in later kinetic studies of the enzyme involving the addition of metals or cofactors often required by other classes of aminomutase, spectroscopic analysis of the wild-type enzyme and the MIO-less variant and correlated $\mathrm{pH}$ profiles with other family members. ${ }^{157}$ The structure of the enzyme was also solved confirming both the presence of the fully formed MIO in the active site and the structural homology of this protein with characterized aromatic amino acid ammonia-lyases. ${ }^{158}$

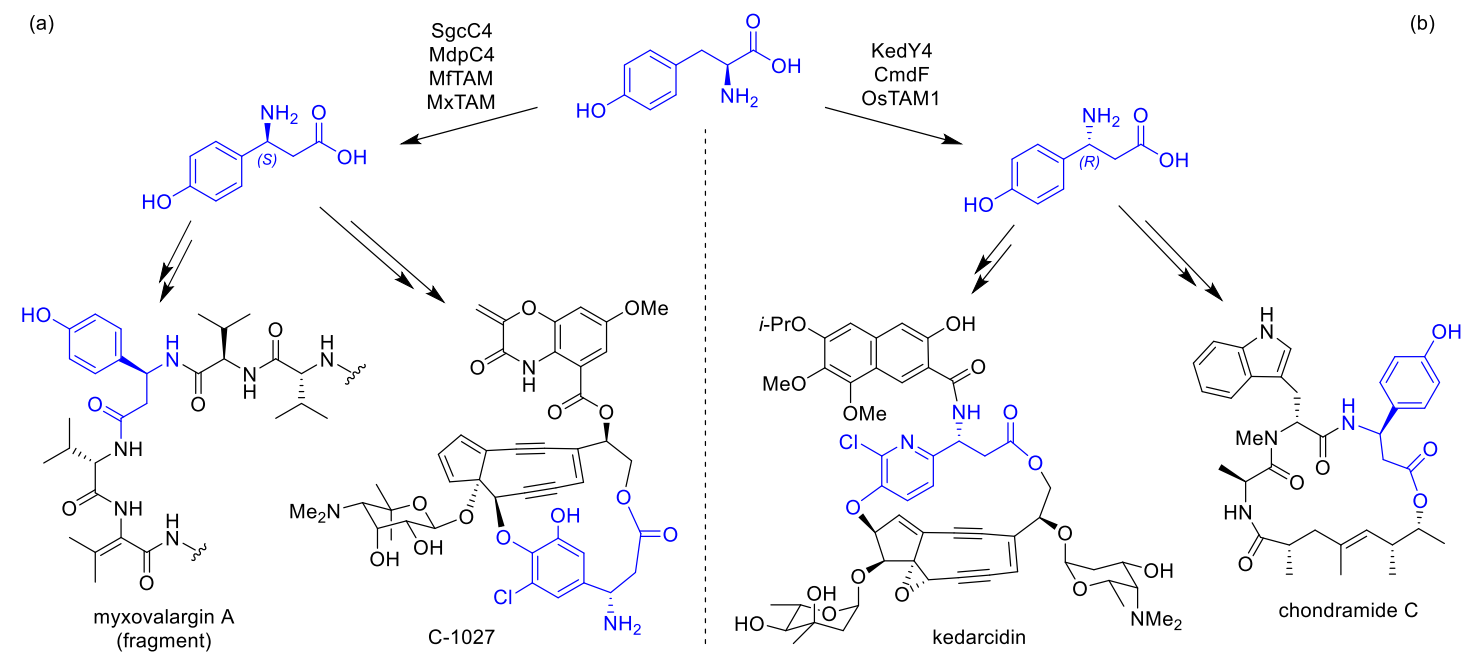

Scheme 12. Examples of enzymes with $(S)$-TAM activity (a) and $(R)$-TAM activity (b), implicated in secondary metabolic processes.

Following this first example of a MIO-dependent aminomutase, other family members were uncovered in related and more diverse metabolic pathways. One example of this is the $\mathrm{SgcC} 4$ orthologous protein MdpC4 from Actinomadura madurae, another $(S)$-TAM involved in the production of the structurally similar enediyne natural product madurapeptin. ${ }^{159}$ Another more recent discovery was the enzyme KedY4 from Streptoalloteichus sp., an $(R)$-selective family member that was predicted to be involved in the production of the 2-azatyrosine derivative central to the kedarcidin toxin (Scheme 12b). By again tracing the $s g c$ gene cluster template onto this more unusual example of an enediyne antibiotic, the putative activity of this orthologue was 
confirmed $^{160}$ constituting the first discovery of a class I lyase-like enzyme with a nonproteinogenic amino acid as its natural substrate.

Until the discovery of KedY4, the only example of an $(R)$-selective aminomutase from bacteria was CmdF from Chondromyces crocatus (also referred to as CcTAM). This enzyme was found to be involved in secondary metabolic synthesis of cytotoxic depsipeptides called chondramides, previously known to contain $(R)-\beta$-tyrosine (Scheme $12 \mathrm{~b}) .{ }^{161,162}$ Interestingly, when this enzyme was used as a query sequence for the discovery of new family members, none of the hits shared similar catalytic activity with $\mathrm{CmdF}$. Those most closely related were found to be $(S)$-TAMs from the Myxococcus genus of bacteria (MfTAM from M. fulvus and MxTAM from an unnamed species) and possibly implicated in non-ribosomal peptide synthesis of the $(S)$ - $\beta$-tyrosinecontaining antibiotic myxovalargin A (Scheme 12a). ${ }^{134}$ This observation, combined with the enantiocomplementarity of KedY4 and McpC4/SgcC4, suggests that enantioselectivity switching between closely related family members involved in similar biosynthetic pathways has led to convergent evolution of $(S)$-TAM activity, rather than transfer of $(S)$-TAM encoding genes between distantly related bacteria.

Another relative found through the same database search was the homologous enzyme from Cupriavidus metallidurans. Despite discovery of weak $(R)$-TAM activity in this enzyme, the deamination product $p$-coumarate was found in vast excess in all cases making it a probable TAL under physiological conditions. ${ }^{134}$ The interesting distribution of enantioselectivity among the bacterial TAM clades has prompted mutational studies, ${ }^{134}$ mechanistic, labelling and $\mathrm{pH}$ profile studies $^{163}$ of the $(R)$-selective CmdF. The results are in accordance with the incidence of $\beta$ tyrosine racemase activity unveiled by earlier kinetic studies of the enantiocomplementary SgcC4. ${ }^{157}$ As such, it is possible that this is a feature of all TAMs as an evolutionary artefact of many selectivity switching events between $(R)$ - and $(S)$-specific enzymes.

There has been evidence for a long time that eukaryotes also harbor enzymes with TAM activity, e.g., the detection of such activity in the purple fungus Cortinarius violaceus in the absence of 
characterisation of the specific protein responsible. ${ }^{164}$ The most recent addition to the suite of class I lyase-like enzymes with TAM activity is an $(R)$-selective enzyme (OsTAM1) from the rice plant Oryza sativa. Its discovery was prompted by the finding of $(R)-\beta$-tyrosine production by rice plants in response to jasmonate exposure, possibly as a counter defense mechanism, given the concurrent finding that the non-natural amino acid product inhibits growth of some competing species. ${ }^{165}$ This information, combined with previous studies of aminomutases within the class I lyase-like family of enzymes, allowed identification of a candidate open reading frame whose product was revealed to be a TAM, ${ }^{165}$ the first example of such an enzyme in eukaryotes. Further biochemical studies revealed that, unlike its bacterial relatives, OsTAM1 did not display decreased or variable enantioselectivity, giving consistently high enantiomeric excess for the $(R)$ configured product even after prolonged reaction time and over a range of $\mathrm{pH}$ values. The enzyme also showed acceptance of phenylalanine, albeit with an activity just $3 \%$ of that for tyrosine, ${ }^{166}$ which possibly points to the evolutionary origin of this enzyme from an ancestral plant PAL. Building on this, variations identified at two positions in the active site of OsTAM1, and the related phenylalanine-specific TcPAM, ${ }^{166}$ allowed mutagenic studies of the enzyme to increase binding affinity for phenylalanine. ${ }^{167}$ Interestingly, the variation of either (but not both) of these residues served not only to increase phenylalanine acceptance, but also to enhance overall turnover and ammonia-lyase activity. ${ }^{167}$ This alteration between TAM- and PAL-like activity is reminiscent of studies with PcPAL-Glu484Asn, where decrease in catalytic efficiency with L-phenylalanine and increase with L-tyrosine was observed. ${ }^{168}$ These results taken together highlight the importance of fine-tuning mutations for substrate specificity which occur at positions other than the selectivity residues.

Apart from OsTAM1, with its PAM side-activity, there also exist a suite of distinct aromatic amino acid aminomutases active on L-phenylalanine, whose biological function is to provide a pool of $\beta$-phenylalanine for incorporation into various natural products. The most investigated of these are the $(R)$-PAM orthologues ubiquitous across taxol-producing species of yew tree. This 
group of nearly identical proteins ${ }^{169-171}$ have received much attention due to their mediation of the first committed step in the biosynthesis of the side chain of taxol, ${ }^{171}$ the most well-known bioderived anticancer drug in clinical use. ${ }^{172}$ The existence of this class of enzymes in many of these yew tree species, as well as several taxol-producing fungi, is often anecdotal through presence of the final product. ${ }^{173}$ One of the first studies to provide direct evidence of aminomutase activity was in a cell-free assay of Taxus brevifolia. ${ }^{174}$ This discovery laid the groundwork for investigation of other enzymes from Taxus species, such as T. cuspidata, T. chinensis and T. wallichiana (with extremely high sequence identity), which have each been studied as isolated proteins and their catalytic activity confirmed. ${ }^{169-171}$ Two of these in particular, from $T$. canadensis (TcPAM) and from T. wallichiana var. chinensis (TwPAM), have subsequently been investigated in great detail due to the unusual stereochemical requirements of the $(R)$-PAM reaction.

While the formation of $(S)$ - $\beta$-phenylalanine through MIO chemistry proceeds with deamination and reamination from the same stereoheterotopic face of the substrate (Scheme 13a), the production of $(R)$ - $\beta$-phenylalanine from the corresponding $(S)$-configured proteinogenic regioisomer necessitates abstraction of the amino group from one face of the newly formed cinnamate intermediate, and reamination on the opposite face (Scheme 13b). Upon investigation using labelling studies and TcPAM, it was also revealed that the concomitant proton transfer is mediated in a similar but opposite fashion, constituting in a formal exchange with the amine group from the opposite face. ${ }^{175}$ Kinetic isotope effects of the labelled and unlabelled substrates from the same study highlighted the initial proton extraction as the rate limiting step in the catalytic cycle. ${ }^{175}$ The method by which the enzyme accomplishes this rearrangement has been subject to speculation, with suggestions including reorientation of the entire ligand in the active site $^{176}$ and possible conformational changes of the intermediate involving rotation around both the $\mathrm{C}_{1}-\mathrm{C}_{\alpha}$ and $\mathrm{C}_{\beta}-\mathrm{C}_{i p s o}$ bonds together ${ }^{170,177}$ or each of these bonds in isolation (Scheme $13 \mathrm{~b}$ ). ${ }^{178,179}$ 
(a) (S)-PAM MECHANISM

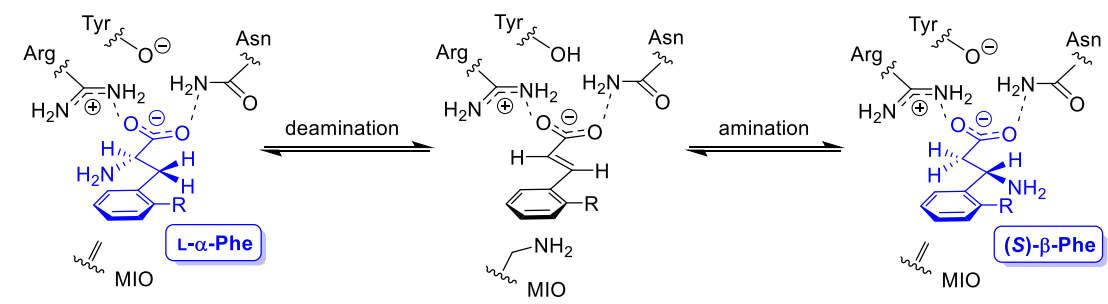

(b) (R)-PAM MECHANISM

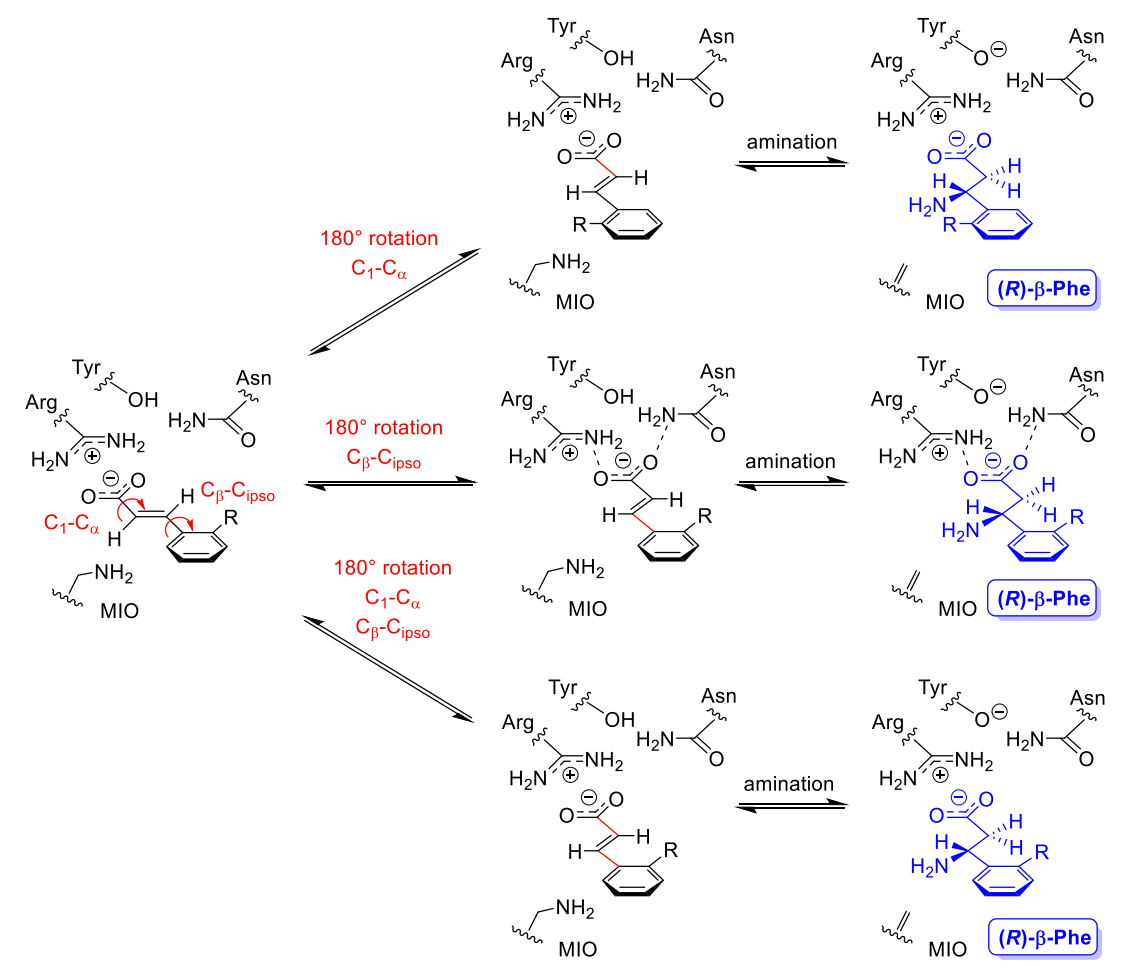

Scheme 13. General deamination-reamination mechanism of $(S)$-selective PAMs (a) and proposed deamination-rotation-reamination mechanism for $(R)$-selective PAMs (b). The bonds around which a rotation is thought to happen are shown in red. The substrate bears a substituent at the ortho-position in order to highlight whether ring flipping has occurred, compared to the previous conformation.

Upon release of the X-ray crystal structure of TcPAM with bound cinnamate ${ }^{170}$ it could be seen that the reaction intermediate was accommodated in a non-planar form, with distortion of bond angles around the central $\pi$-system of the molecule and a misalignment of the aryl ring and carboxyl group in their respective active site binding pockets. This could be seen to imply an 
enzyme mediated partial disruption of the conjugation of the reaction intermediate, decreasing the torsional barrier of rotation around both $\mathrm{C}_{1}-\mathrm{C}_{\alpha}$ and $\mathrm{C}_{\beta}-\mathrm{C}_{i p s o}$ bonds, whilst also providing minimal perturbation of the two conformers with regard to pre- and post-rotational positioning of the aromatic ring. Interestingly, in the study citing conformer interchange involving torsion at both bonds, the reaction is seen not to proceed with a non-natural substrate which would result in a 2methylcinnamate intermediate. ${ }^{177}$ Assuming rotation around both bonds, one might predict that this substrate would provide only minor hindrance to the presentation of either stereoheterotopic face of the central double bond compared with the unsubstituted intermediate, whereas rotation around just the $\mathrm{C}_{1}-\mathrm{C}_{\alpha}$ would, as it involves flipping of the entire ring. This is a hypothesis backed up by computational studies of the same enzyme where calculation results indicate not only preference for a single $180^{\circ}$ rotation around the $\mathrm{C}_{1}-\mathrm{C}_{\alpha}$ bond, but also a favouring of the MIOadduct mechanism and ammonia elimination through an E2 mechanism. ${ }^{179}$ The in silico study has also predicted that the MIO abstraction/addition of the amino group could only occur at the $\alpha$ position in one of the two conformers and at the $\beta$-position in the second conformer, assuming a single bond rotation. ${ }^{179}$ This is consistent with the established strict enantioselectivity of the rearrangement reactions for this enzyme, ${ }^{175}$ as it contravenes the production/conversion of both D-phenylalanine and $(S)-\beta$-phenylalanine in theory. The most recent structural studies, this time of the closely related TwPAM, however, provide evidence for two distinct binding modes of the cinnamate intermediate in the active site, ${ }^{176}$ possibly mitigating the need for any sort of bond rotation. With the earlier realization that the plant $(R)$-PAM and PAL active sites are nearly identical, differing by only a single aryl binding pocket residue, ${ }^{180}$ the molecular mechanism by which $(R)$ - $\beta$-selective ammonia readdition is enabled uniquely in the Taxus-derived enzymes remains unclear, regardless of the precise mode by which the stereoheterotopic faces of the central double bond are presented to the MIO and catalytic tyrosine. It is likely that the residues that determine this lie elsewhere in the protein, perhaps altering breathing motions to reshape the active site of PAMs during catalysis. ${ }^{176}$ 
Despite the PAMs from Taxus sp. being the only known $(R)$-selective and phenylalanine specific mutases for a long time, there have always been clues hinting at the existence of analogous enzymes in other organisms (Scheme 14a). Such evidence includes the occurrence of other metabolites containing ( $R$ - $\beta$-phenylalanine, the most notable examples of these being the chemically similar astins and cyclochlorotines from the plant Aster tartaricus and fungus Talaromyces islandicum respectively. ${ }^{181,182}$ Although these bioactive pentapeptides were isolated and characterized decades ago, knowledge of their biosynthesis was hindered by the lack of genome sequence data. With the recent release of the $T$. islandicum genome, ${ }^{183}$ the biosynthetic gene cluster associated with cyclochlorotine production could be identified. ${ }^{184}$ One of the putative genes within the group was predicted to code for a member of the class I lyase-like family and, through knowledge of the aminomutase potential of MIO catalysis and previous evidence of convergent evolution of mutase activity, it has been reasoned that the gene product, CctP, may well be an $(R)$-PAM responsible for the $(R)$ - $\beta$-phenylalanine found in the final natural product. ${ }^{184}$ Interestingly, the similarity between the astin and cyclochlorotine compounds extracted from members of two separate eukaryotic kingdoms minimizes the likelihood that these organisms have arrived at the same pentapeptide structure by chance and sheds light on the possibility of horizontal gene transfer or endophytic production of the astin series by a fungal plant symbiont. Upon further investigation the latter seems to have been the case, as seen in recent work on the characterization of the Aster-associated, astin-producing fungus Pelliciarosea asterica. ${ }^{185}$ Given the close relationship between this organism and species of Talaromyces, ${ }^{185}$ it stands to reason that $P$. asterica also harbors an $(R)$-PAM making fungi a possibly rich source of new enzymes with this activity. 


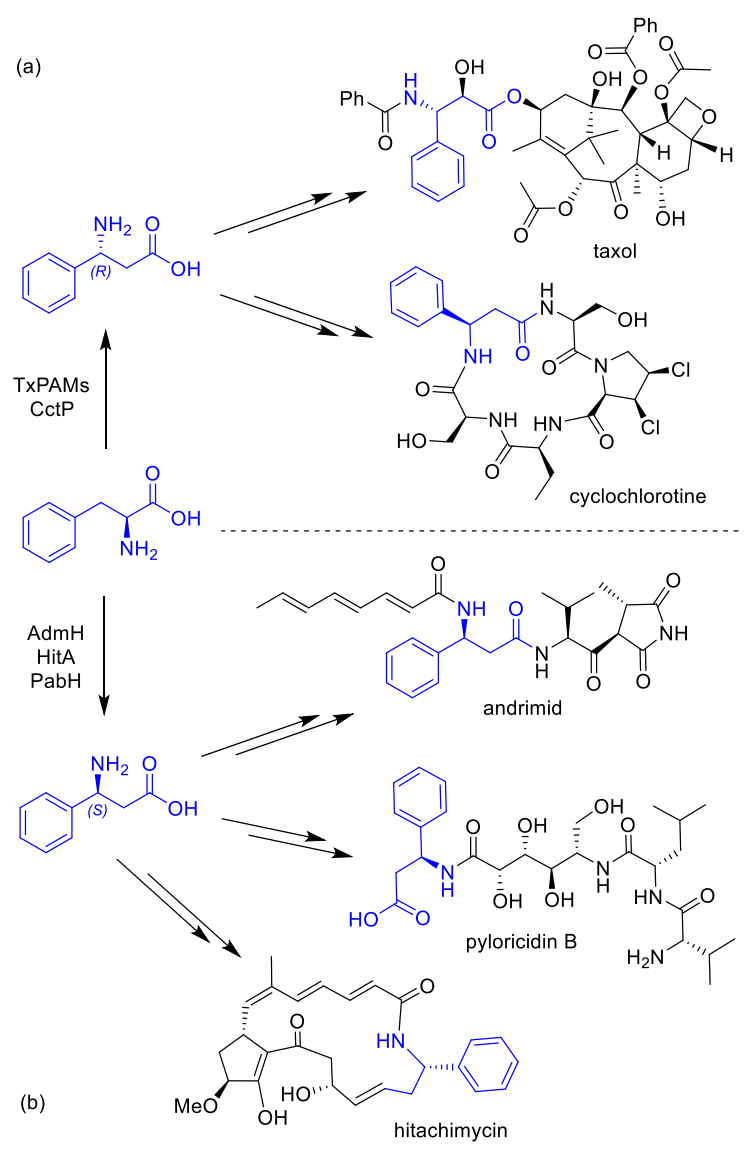

Scheme 14. Examples of enzymes with (R)-PAM activity (a) and (S)-PAM activity (b), implicated in secondary metabolic processes.

Unlike the $(R)$-selective PAM enzymes, their enantiocomplementary relatives are only known from various examples in the eubacterial kingdom (Scheme 14b). The first of these to be discovered, and thus most extensively characterized, was AdmH from Enterobacter agglomerans, which was identified in the context of the biosynthetic gene cluster for the antibiotic andrimid. ${ }^{186}$ Later studies confirmed the excellent $(S)$-selectivity of this enzyme, a feature linked to the natural gatekeeping mechanism of the biosynthetic process ensuring chemical fidelity and final product homogeneity when the gene cluster is upregulated. ${ }^{187}$ The discovery of a PAM with opposite enantioselectivity to the previously characterized Taxus enzymes prompted mechanistic studies and comparisons between the two isomerization reactions. Whereas TcPAM from the Canadian 
yew tree was expected to mediate rotation of the acrylic acid intermediate in the active site, labelling studies showed this need not be the case for AdmH, with both proton/amine abstraction and re-addition occurring on the same respective faces. ${ }^{177}$ Further evidence was added to this by parallel isomerization assays with both enzymes using L-2-methylphenylalanine as a chemical mechanistic probe. The turnover of this substrate to the expected regioisomer by AdmH, but not by TcPAM, was cited as evidence for the lack of a torsional barrier to isomerisation in the former enzymatic process. ${ }^{177}$ Finally, confirmation of this binding mode was achieved through crystallographic studies of the enzyme, revealing MIO-amino acid adduct electron densities consistent with both $\beta$ - and $\alpha$-phenylalanine derivatives from separate active sites. ${ }^{188}$ The minimal shift in backbone position seen between the $\beta$ - and $\alpha$-regioisomers in overlaid structures indicates that the AdmH mediated binding mode of the substrate allows the mutase reaction to occur without repositioning and/or major torsional considerations.

The structural insight from the studies of AdmH also allowed rationalization of the enzyme's unique substrate positioning, with this being attributed to a bulky residue (Phe455) not found in any other active site of a known class I lyase-like enzyme. The size and position of this residue was seen to alter the trajectory of substrate binding, making it similar to that employed by the $(S)$ TAM SgcC4, although with a different molecular basis. ${ }^{188}$ This finding was used to uncover homologous sequences with the same residue at aligning positions and putatively assign them $(S)$ PAM activity. Initially, six putative sequences were found, ${ }^{188}$ but owing to ongoing genomic sequencing efforts the same search repeated more recently uncovered 19 hits. One of these, PabH from a species of the genus Bacillus, could be tentatively attributed to the biosynthesis of pyloricidin-like antibacterial compounds via a genetic context analysis and, upon characterization, was also found to possess the predicted aminomutase activity. ${ }^{189}$ The only other instance of an (S)-PAM known to be involved in secondary metabolism is the case of HitA from Streptomyces scabrisporus. In this example, the biosynthetic gene cluster for the known polyketide hitachimycin was identified from the genome sequence of the host organism solely 
based on the presence of a PAM-like sequence. Rather than looking for particular sequence motifs, the entire query sequence of a known PAM was used to identify a potential open reading frame in the context of probable polyketide biosynthetic genes. Upon characterization of the putative aminomutase, $(S)$-PAM activity was confirmed, as required to achieve the correct stereochemistry for the natural product under investigation. ${ }^{190}$

Another of the enzymes selected in both of the aforementioned database searches for its possession of an (S)-PAM-like active site was EncP from Streptomyces maritimus. ${ }^{188}$ Unlike the other hits, which were all uncharacterized sequences, this enzyme was already known to be involved in the production of wailupemycin metabolites and the antibiotic enterocin in its host organism, courtesy of its PAL activity. ${ }^{191}$ Upon further characterization, however, the enzyme was found to exhibit very low turnover compared to other known ammonia-lyases and to have the highest sequence similarity to the mutase AdmH. ${ }^{192}$ As an investigation of this, both enzymes were tested for ammonia-lyase and aminomutase activity over a range of temperatures, to reflect the different physiological environments they would be subjected to in the thermotolerant $S$. maritimus and mesophilic E. agglomerans. Strikingly, both enzymes were found to exhibit mutase activity at lower temperatures with only minimal production of cinnamate when Lphenylalanine was used as a starting material. ${ }^{193}$ This finding was in accordance with the leaking of cinnamate reported upon the first biochemical characterization of $\mathrm{AdmH}^{187}$ and also explains in part the apparently low reaction rate for EncP in initial studies, ${ }^{192}$ where only formation of the ammonia-lyase product cinnamate was measured. At higher temperatures, both enzymes were seen to produce an increasing amount of cinnamate, with minimal PAM activity detectable at temperatures of around $70^{\circ} \mathrm{C}$ (Scheme 15). This thermal bifunctionality between PAM and PAL activity was reasoned to be due to the effect of increased temperature on the dynamics of the enzyme. Whilst at lower temperatures the inner active site loop would remain in the closed conformation long enough for deamination and reamination to occur, at higher temperatures interruption of the catalytic cycle (with loop opening and release of the intermediate) would result 
in ammonia-lyase activity. ${ }^{193}$ From a physiological perspective, knowledge of the distinct pathways associated with EncP and AdmH allows speculation that, whilst both enzymes possess unwanted side activities, they are unlikely to have detrimental wasteful effects in vivo. This is because AdmH has been shown on two accounts to produce only a small amount of unproductive cinnamate $^{187}$ and EncP, whilst able to form a sizable amount of $(S)-\beta$-phenylalanine, can also revert it to metabolically active L-phenylalanine or deaminate it to cinnamate ${ }^{193}$ under the same conditions. In this way the relevant product can be siphoned off into the host specific pathway allowing the bifunctional PAL/(S)-PAM enzymes to act as multipurpose starting enzymes for different metabolic strategies in an evolutionary context.

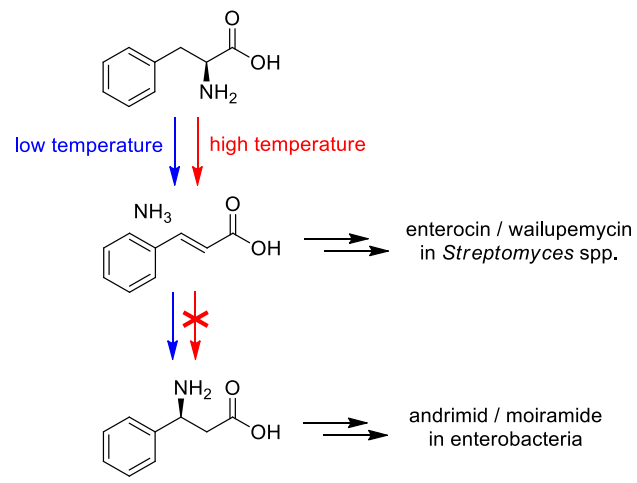

Scheme 15. The thermobifunctionality of EncP and AdmH which both display predominantly $(S)$ PAM activity at low temperature and more PAL activity as the temperature increases.

As briefly demonstrated in this section, PAMs and TAMs occupy a key position in secondary metabolism to provide adequate supplies of aromatic $\beta$-amino acids for the incorporation in a strikingly broad range of complex natural products. Likewise, their activity has been exploited in preparative synthesis not only to afford these valuable natural precursors, but also multiple related non-natural building blocks (e.g., for medicinal chemistry), as will be discussed in Section 4.4. 


\section{Synthetic applications of ammonia-lyases and aminomutases}

The past few decades have seen an exponential rise in the use of biocatalysts to convert readily available and inexpensive starting materials to value-added products. This has been credited to several factors, including the advancement in recombinant DNA technologies, the development of suitable high-throughput screening systems, the improvement of bioinformatics for the discovery of new enzymes, and the reduction in the cost of DNA synthesis and sequencing. This has led to the identification and development of enzymes with unique and novel chemistry better suited for sustainable manufacturing. Perhaps the greatest influence came from the fine chemical and pharmaceutical industry at a time when strict rules and regulations were enforced to reduce the carbon footprint in order to combat climate change. This led to the adoption of greener strategies to mitigate current problems the industry was facing, including the need for harmful organic solvents, the generation of toxic waste, the poor atom economy of most fine chemical processes and the reliance on non-renewable feedstocks. The concept of "greenness" of chemical processes was already considered relevant in the 1960s, however, it was only in 1998 when Anastas and Warner developed the 12 principles of green chemistry. Biocatalytic processes typically adhere to most (if not all) of these principles, contributing to the ever-increasing popularity of enzymatic reactions in industrial processes. In this section, multiple examples of ammonia-lyase and aminomutase biocatalytic processes (including chemo-enzymatic and multienzymatic cascades) will be provided, highlighting the advantages brought to chemical manufacturing.

\subsection{Synthesis of aspartic acid derivatives}

Undoubtedly, aspartase represents one of the earliest success stories of biocatalysis for industrial applications. Its high specificity and activity were immediately recognized and exploited in several industrial processes for the preparation of L-aspartic acid from fumaric acid and ammonium salts, comprehensively reviewed elsewhere. ${ }^{1,3}$ The earliest applications employed 
wild-type $E$. coli cells immobilized on various supports, such as polyacrylamide, ${ }^{194}$ polyurethane ${ }^{195}$ or carrageenan, ${ }^{196}$ with high productivity, good stability and, most importantly, low cost. Subsequent improvements on this process involved the use of recombinant cells overproducing aspartase ${ }^{197}$ and the application of thermotolerant DALs with increased stability under the process conditions. ${ }^{198}$ On laboratory scale, the aspartase reaction has been demonstrated also for the enantioselective synthesis of specifically labelled L- $\left[1,4-{ }^{13} \mathrm{C}_{2}\right]$-aspartic acid from $[1,4-$ ${ }^{13} \mathrm{C}_{2}$ ]-fumaric acid (44\% isolated yield). ${ }^{199}$

A number of technical solutions have been reported to improve conversions and/or overall efficiency of the aspartase reaction. In particular, a recent strategy based on the overproduction of AspA in the psychrophilic organism Shewanella livingstonensis Ac10, led to the improvement of conversions to $99.3 \%$. The cells were heat-treated to deactivate most of the endogenous enzymes (including fumarase, which interferes with the aspartase reaction producing malate as a byproduct). ${ }^{200}$ Several systems were also patented for the chemical recycling of the waste stream from the aspartase reaction. The use of maleic anhydride to lower the $\mathrm{pH}$ of the L-aspartate solution and precipitate the L-aspartic acid generates an effluent containing only maleic acid and low concentrations of ammonia. Isomerization of maleic acid to fumaric acid (by treatment with ammonium bromide and ammonium persulfate, followed by ion-exchange) and $\mathrm{pH}$ adjustment (by addition of ammonium hydroxide) affords a solution of fumaric acid that can be directly recycled. ${ }^{201}$ A more elegant system, perfecting the same concept, involves the coupling of aspartase with maleate isomerase, to perform the isomerization of maleate to fumarate in the same reaction system as the addition of ammonia. ${ }^{202}$ This makes the whole process more efficient, avoids the additional chemical step and affords the aspartate product in much higher purity (Scheme 16). 


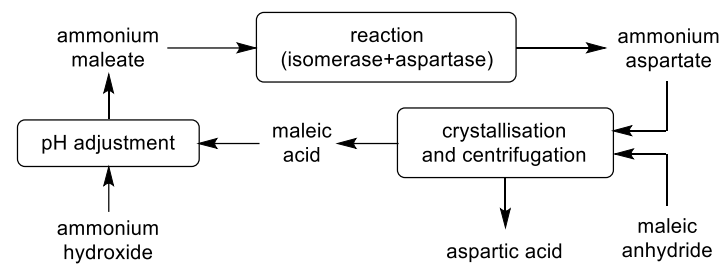

Scheme 16. Coupling of aspartase and maleate isomerase for the industrial-scale synthesis of Laspartate with waste stream recycling.

Aspartase has also been employed in several other multi-enzymatic cascade processes, since its high substrate specificity prevents cross-reactions with other enzymatic systems. One example is the recent co-immobilisation of $\mathrm{AspB}$ with a microbial aspartate transaminase (AspTA) to produce L-phenylalanine from phenylpyruvate, using as the amino donor L-aspartate formed in situ from fumarate (Scheme 17a). ${ }^{203}$ The two enzymes were co-immobilized on an amino-epoxy support, giving a 95\% yield of L-phenylalanine at $100 \mathrm{mM}$ concentration in continuous flow. In another example, Zhu and co-workers employed aspartase as an ammonia scavenger to shift the equilibria of a nitrilase reaction for the production of $\beta$-alanine (Scheme 17b). ${ }^{204}$ The reaction of 3-aminopropionitrile with a nitrilase identified from a screen (NIT3397 from Bradyrhizobium japonicum) affords $\beta$-alanine and ammonia, with traces of the intermediate 3 -aminopropanamide. Upon increasing the substrate concentration up to $3 \mathrm{M}\left(210 \mathrm{~g} \mathrm{~L}^{-1}\right)$, the accumulation of considerable quantities of amide was observed, because of the equilibrium established between the acid, the amide and ammonia in the enzymatic hydrolysis. The addition of DAL and fumarate to the system, to shift the equilibria by consuming the by-product ammonia, resulted in almost complete conversion of the amide to acid with no influence on the nitrilase activity. 

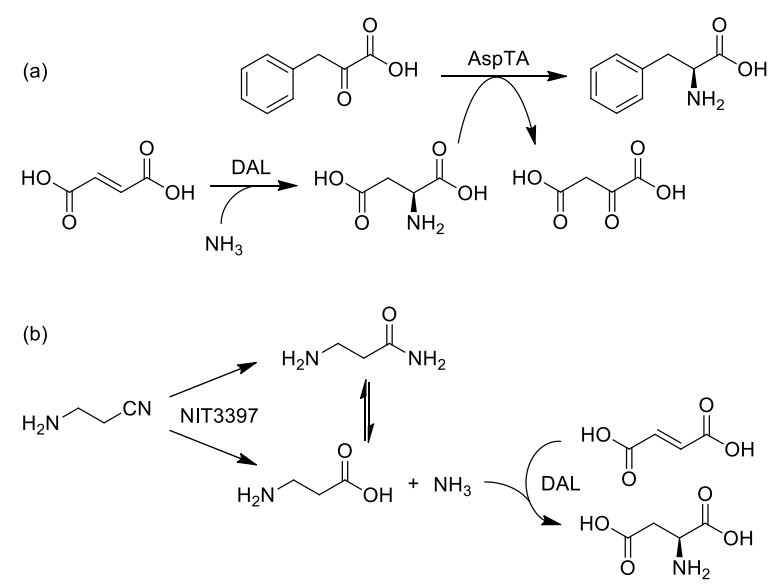

Scheme 17. Examples of cascade applications of DALs: (a) in situ generation of a co-substrate; (b) in situ removal of a by-product to drive an enzymatic equilibrium reaction.

Another remarkable cascade application of aspartase was as part of an in vitro artificial metabolic cycle for the interconversion of chemical functional groups employing six different enzymes (Scheme 18): aspartase AspB from Bacillus sp. (DAL), L-aspartate oxidase from Sulfolobus tokodaii (LASPO), L-malate dehydrogenase from bovine heart (LMDH), and fumarase from porcine heart (FUM), combined with commercial catalase (CAT) and formate dehydrogenase (FDH) from unspecified bacterial sources. ${ }^{205}$ The four main steps of the cycle involve the conversion of fumarate to L-aspartate (by DAL), dehydrogenation to 2-oxaloacetate (by LASPO coupled with CAT to break down $\mathrm{H}_{2} \mathrm{O}_{2}$ ), reduction to L-malate (by LMDH coupled with FDH to regenerate the NADH cofactor) and dehydration (by FUM), regenerating fumarate ready to start a new cycle. The authors demonstrated that the addition of any of the intermediates to this multienzymatic system establishes a steady state in which concentrations of all the four compounds do not vary and the oxidation of formate at the expense of molecular oxygen proceeds continuously. This process exemplifies the concept of systems biocatalysis, where simple and modular enzymatic activities are combined to achieve the desired transformation. 


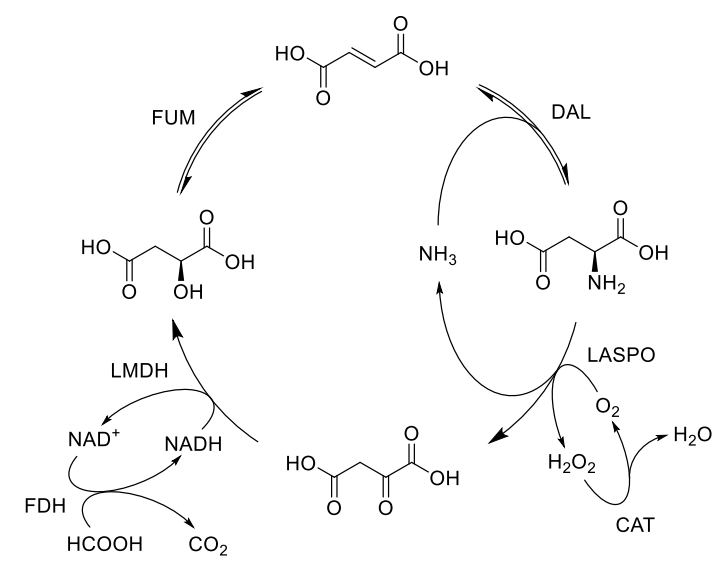

Scheme 18. In vitro artificial cascade cycle for the interconversion of functional groups, as an example of artificial metabolism. The net reaction consumes formate and oxygen and produces water and carbon dioxide.

Apart from the plethora of uses of aspartase for the natural (forward or reverse) reaction, the expansion of its application to the synthesis of substituted aspartic acid derivatives has been considered highly desirable as well. Many aspartate derivatives are building blocks of bioactive compounds such as pharmaceuticals and natural products, and their chemical synthesis remains quite challenging. However, as mentioned in Section 2.1, aspartases were found to be highly specific for their natural reaction, in spite of countless attempts to find alternative substrates. The first example of promiscuity of the aspartase from Bacillus cadaveris was reported by Emery in 1963: the synthesis of $N$-hydroxyaspartic acid by reaction of fumarate with hydroxylamine as the nucleophile. ${ }^{206}$ The product was identified by its physico-chemical properties, but proved too unstable to isolate. Only in 2008 was a similar experiment attempted and expanded in order to probe the specificity of AspB for the nucleophile. ${ }^{207}$ Incubating the enzyme with fumarate and a panel of several small nucleophiles gave complete conversion not only with hydroxylamine, as reported by Emery, but also with hydrazine, methoxyamine and methylamine (Scheme 19a). Larger (ethylamine, glycine, formamide) and charged (azide, cyanide, cyanate) nucleophiles afforded no conversion. The isolation problems with $N$-hydroxyaspartic acid could not be solved 
by purification, protection or derivatization, but all the other derivatives were easily purified by ion-exchange chromatography. ${ }^{207}$

(a)

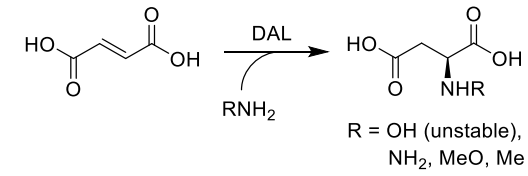

(b)

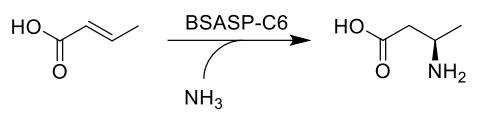

Scheme 19. Synthesis of various amino acids with wild-type and mutant DALs: $N$-substituted aspartic acid derivatives with AspA and AspB (a) and (R)-3-aminobutyrate (b).

The consideration that the $\beta$-carboxylate group of the substrate plays a key role in stabilizing the intermediate, while the $\alpha$-carboxylate is not actively involved, prompted Vogel et al. to start an engineering project to alter the active site of AspB to accommodate different functional groups. ${ }^{208}$ Four positions in close contact with the $\alpha$-carboxylate were selected and simultaneously mutated (Thr187, Met321, Lys324, Asn326), affording a considerably large library. The screening of 300,000 clones against methyl crotonate (by HPLC, using cluster screening in mixed populations) afforded the quadruple mutant Thr187Cys-Met321Ile-Lys324Met-Asn326Cys, named BSASPC6, active on the selected substrate to form $(R)$-3-aminobutyrate (Scheme 19b). Even though the catalytic performance was poor, this is the first example of active site reshaping of a DAL, an exciting proof-of-concept of the applicability of these enzymes to a broader set of products. This also highlights the importance of synergistic mutations (further analysis revealed that at least three mutations were decisive) and the effectiveness of cluster screening for large library sizes.

Concerning MALs, instead, their reaction on natural substrates is of limited interest for synthetic applications. The use of CtMAL to prepare L-threo-3-methylaspartic acid (from mesaconate, $76 \%$ yield), ${ }^{92}$ and D-threo-3-methylaspartic acid (from the racemic threo-diastereoisomer, by removal of the L-enantiomer, $36 \%$ yield $)^{93}$ is obviously very efficient and has been described 
already in the very early studies. However, in contrast with DALs, the real potential of MALs lies in their activity on non-natural substrates, which was recognized early as well.

The ability of CtMAL to accept various $\beta$-substituted aspartic acids (ethyl, $n$-propyl, isopropyl side chains with progressively low rates, while no activity was observed with $n$-butyl) was reported in $1967,{ }^{209}$ but the first reports of asymmetric additions on substituted fumaric acids appeared only about two decades later. Gani and co-workers first tested a panel of 2halofumarates as substrates, obtaining different results: with 2-fluorofumarate the reaction occurred slowly with accumulation of multiple side-products; with 2-chlorofumarate the product could be isolated in high yield and purity; with 2-bromofumarate the product was formed efficiently but could not be isolated due to rapid cyclization to the corresponding 2,3aziridinedicarboxylate, and irreversible inhibition of the enzyme. ${ }^{210,211}$

They also demonstrated the synthesis of a panel of 3-alkylaspartates with CtMAL, starting from 2-alkylfumarates (Scheme 20), including specifically ${ }^{2} \mathrm{H}$-labelled aspartates obtained by running the reactions in $\mathrm{D}_{2} \mathrm{O} .{ }^{212}$ The same group also reported the synthetic access to $N$-substituted aspartate derivatives using other nitrogen nucleophiles (e.g., hydrazine, methylamine, hydroxylamine, methoxyamine). ${ }^{213}$ Even though this work showed a much greater substrate tolerance than aspartases (Scheme 20), the reaction is still limited to small nucleophiles. A similar substrate scope was also reported later for the thermostable ChMAL. ${ }^{100}$ 


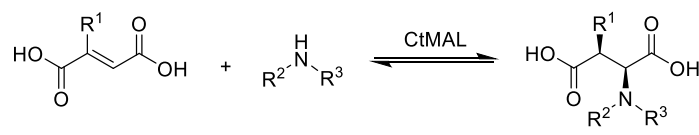

\begin{tabular}{l|ccccc|} 
Isolated yield [\%] & $\mathrm{R}^{1}=\mathrm{H}$ & $\mathrm{R}^{1}=\mathrm{Me}$ & $\mathrm{R}^{1}=\mathrm{Et}$ & $\mathrm{R}^{1}=n-\mathrm{Pr}$ & $\mathrm{R}^{1}=i-\mathrm{Pr}$ \\
\hline $\begin{array}{l}\text { ammonia } \\
\mathrm{R}^{2}=\mathrm{R}^{3}=\mathrm{H}\end{array}$ & 90 & 61 & 60 & 49 & 54 \\
$\begin{array}{l}\text { hydrazine } \\
\mathrm{R}^{2}=\mathrm{NH}_{2}, \mathrm{R}^{3}=\mathrm{H}\end{array}$ & 42 & 61 & 57 & 31 & 33 \\
$\begin{array}{l}\text { methylamine } \\
\mathrm{R}^{2}=\mathrm{Me}, \mathrm{R}^{3}=\mathrm{H}\end{array}$ & 45 & 40 & 35 & n.r. & n.r. \\
$\begin{array}{l}\text { dimethylamine } \\
\mathrm{R}^{2}=\mathrm{R}^{3}=\mathrm{Me}\end{array}$ & 28 & n.r. & - & - & - \\
$\begin{array}{l}\text { ethylamine } \\
\mathrm{R}^{2}=\mathrm{Et}, \mathrm{R}^{3}=\mathrm{H}\end{array}$ & $\mathrm{n} . \mathrm{r}$. & - & - & - & - \\
hydroxylamine & & & & & \\
$\mathrm{R}^{2}=\mathrm{OH}, \mathrm{R}^{3}=\mathrm{H}$ & 28 & 19 & 12 & - & - \\
$\begin{array}{l}\text { methoxyamine } \\
\mathrm{R}^{2}=\mathrm{OMe}, \mathrm{R}^{3}=\mathrm{H}\end{array}$ & 31 & 34 & n.r. & - & - \\
\hline
\end{tabular}

Scheme 20. Aspartic acid derivatives prepared with wild-type MAL (n.r. = no reaction).

However, much more striking results were obtained recently by structure-guided engineering, leading to the development of two single-point variants with greatly enhanced nucleophile scope or electrophile scope. ${ }^{214}$ Analyzing the crystal structure of CtMAL with L-threo-3methylaspartate bound, residues Gln73 and Gln172 (in close contact with the amino group) were selected and targeted by site-saturation mutagenesis. The screening for the model reaction of the addition of methylamine to mesaconate led to the identification of Gln73Ala as the variant with the highest catalytic rate. This variant showed a remarkably broad nucleophile tolerance (Scheme 21a), accepting a wide range of amines from simple alkylamines to cyclohexylamine and benzylamine. Remarkably, all the products were obtained with excellent diastereoselectivity. In a similar fashion, from the analysis of the same crystal structure, mutagenesis efforts were then directed to Phe170, Tyr356 and Leu384, that form the binding pocket for the methyl group of mesaconate. Screening for the model reaction of the addition of ammonia to 2-hexylfumarate resulted in the identification of Leu384Ala as the most active variant, which showed an exceptionally broad electrophile tolerance (Scheme 21b), accepting substituted fumarates with a side chain as bulky as a benzyl group, albeit with lower diastereoselectivities across the board. 
Many of these reactions were optimized for preparative scale application, demonstrating the synthetic potential of MAL variants for the synthesis of biologically active compounds. ${ }^{214}$

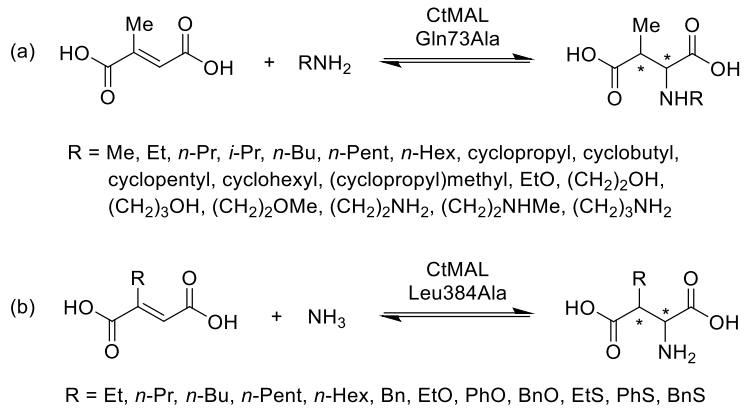

Scheme 21. Expanded nucleophile/electrophile scope of engineered MAL variants.

Lastly, DALs and MALs have also been employed in synthetic biology applications, an area certainly bound to grow in the near future. For instance, the activity of aspartase has been exploited in a recent successful metabolic engineering effort towards the fermentative production of 3-aminopropionic acid ( $\beta$-alanine) from glucose (Scheme 22). ${ }^{215}$ Starting from an engineered E. coli strain producing fumarate as a host, the panD gene from Corynebacterium glutamicum (encoding L-aspartate- $\alpha$-decarboxylase), the aspA gene (encoding aspartase) and the $p p c$ gene (encoding phosphoenolpyruvate carboxylase) were overexpressed either by promoter replacement or using additional plasmids. The final strain allowed production of 3 -aminopropionic acid up to a concentration of $32.3 \mathrm{~g} \mathrm{~L}^{-1}$ over $39 \mathrm{~h}$. 


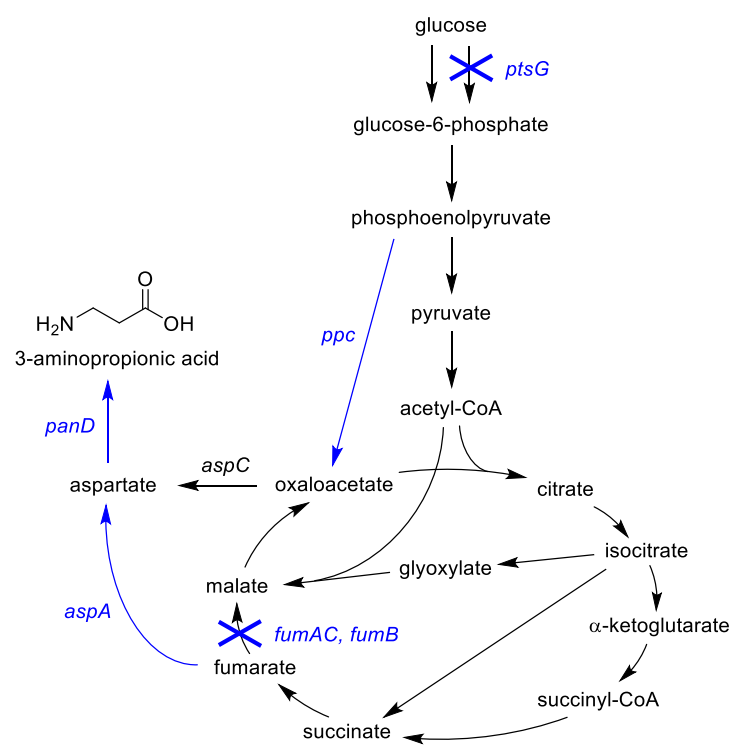

Scheme 22. Metabolic engineering of $E$. coli for the production of 3-aminopropionic acid. The genes overexpressed or deleted in the final strain are shown in blue.

Another recent metabolic engineering project afforded an efficient $E$. coli platform for the production of mesaconate from glucose, with a productivity of up to $7.8 \mathrm{~g} \mathrm{~L}^{-1}$. The constructed pathway includes recombinant expression of glutamate mutase (an AdoCbl-dependent enzyme for the conversion of glutamate to methylaspartate) and MDAL. In order to maximize the efficiency of the glutamate mutase, the two genes encoding for the two subunits of this enzyme were selected from different organisms: the $\operatorname{glm} E$ gene for the dimeric catalytic subunit from Clostridium cochlearium and mutS gene for the monomeric $\mathrm{B}_{12}$-binding subunit from Clostridium tetanomorphum (Scheme 23). ${ }^{216}$ 


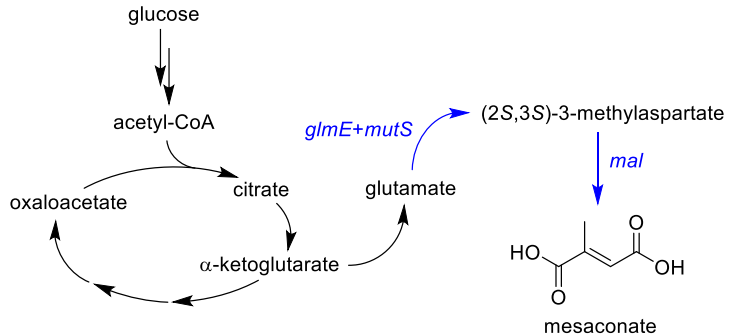

Scheme 23. Metabolic engineering of $E$. coli for the production of mesaconate. The genes overexpressed in the final strain are shown in blue.

\subsection{Synthesis of L-arylalanines}

Derivatives of L-phenylalanine are ubiquitous in nature, involved in many metabolic processes or pathways and are chiral structural units of a number of chemical messengers, including hormones and neurotransmitters. Non-natural L-arylalanines are also crucial in a myriad of chemical processes and are incorporated as key pharmacophores in many active pharmaceutical ingredients (APIs) and drug candidates. Whilst D-phenylalanine is obviously much rarer in nature, the Denantiomers of many arylalanines are almost equally common and useful building blocks in synthetic chemistry. A representative selection of APIs, drug candidates or precursors based on L- and D-arylalanine synthons is presented in Scheme 24. 

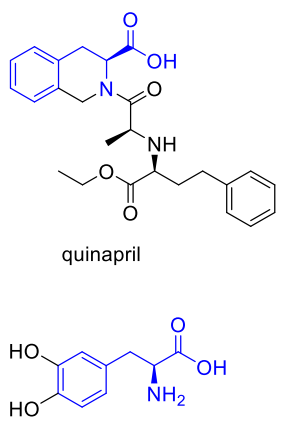

L-DOPA

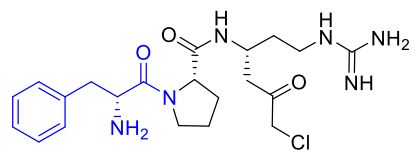

PPACK

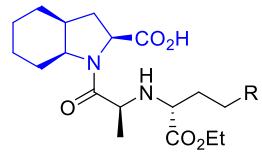

perindopril, $\mathrm{R}=\mathrm{Me}$
indolapril, $\mathrm{R}=\mathrm{Ph}$
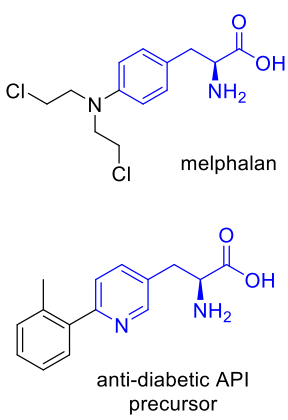
precursor

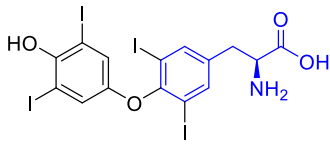

L-thyroxine

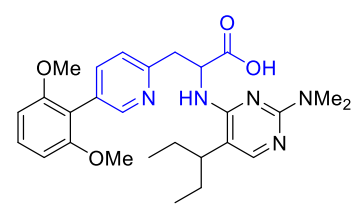

leukocyte adhesion inhibitor

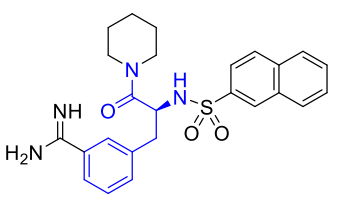

P3APA anticoagulant

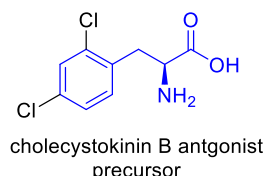

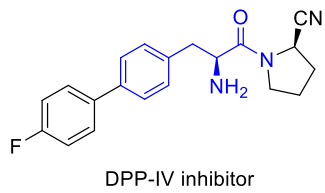

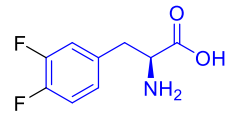

nuclear hormone inhibitor precursor
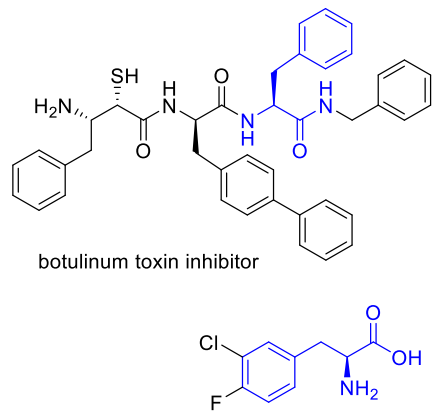

hepatitis $C$ antiviral

Scheme 24. Representative examples of APIs, drug candidates or precursors containing L- and Darylalanine derivatives (the arylalanine moieties are highlighted in blue).

Due to the reversibility of the enzymatic reactions of arylalanine ammonia-lyases, first alluded to by Williams and Hiroms in 1967 for HALs, ${ }^{217}$ and Hanson and Havir in 1968 for PALs, ${ }^{218}$ it is synthetically more useful to drive the reaction in the amination direction to produce the higher value L-arylalanine from the inexpensive arylacrylic acid substrate. Several methods already exist for the chemical synthesis of enantiopure amino acids, for example, the asymmetric hydrogenation of dehydroamino acids, ${ }^{219}$ a process well known for the synthesis of L-DOPA, or the classical Strecker synthesis ${ }^{220}$ (either performed in an asymmetric fashion ${ }^{221}$ or followed by chemical or enzymatic resolution). The ammonia-lyase reaction, however, has a number of unique advantages over other methods: $100 \%$ atom economy, inexpensive and easily accessible substrates, and no requirement for expensive cofactors or regeneration systems. Furthermore, the 
direct addition of ammonia to the $\alpha$-position of the acrylic acid occurs with anti-Michael regioselectivity, a reaction which cannot be emulated with current synthetic methods, highlighting the synthetic utility of these biocatalysts.

In order to favor the equilibrium position towards the amination direction, the biotransformation step is carried out in the presence of high concentrations of ammonia. The potential industrial application of PAL was first demonstrated in 1976, in a patent filed by Pfizer using RtPAL from Rhodosporidium toruloides (formerly known as Rhodotorula gracilis) covalently immobilized onto a solid support. ${ }^{222}$ Unsubstituted cinnamic acid with a concentration of $500 \mathrm{mM}$ in the presence of concentrated buffered ammonia at $\mathrm{pH} 9.5$ gave $90 \%$ yield of L-phenylalanine after 18 h incubation at $37^{\circ} \mathrm{C}$. Yamada et al. soon published the first paper with full optimization parameters of RgPAL from Rhodotorula glutinis for the hydroamination of cinnamic acid to Lphenylalanine. ${ }^{223} \mathrm{~A}$ number of factors were tested, such as optimal growth conditions, $\mathrm{pH}$, ammonia concentration and substrate loading. The best growth conditions were found with $1 \%$ yeast extract, $1 \%$ peptone, $0.05 \%$ L-phenylalanine and $0.5 \%$ sodium chloride. Substrate loading experiments showed $60 \mathrm{mM}$ to be the ideal concentration for amination (concentrations higher than this resulted in substrate inhibition). Optimum ammonia concentration was determined to be $9 \mathrm{M}$ at $\mathrm{pH} 10.0$, yielding $70 \%$ conversion to L-phenylalanine.

Earlier work on the synthesis of L-phenylalanine opened up new avenues towards the synthesis of non-natural amino acids. It was a decade later when RgPAL was screened against a comprehensive panel of substituted cinnamic acid derivatives, highlighting the scope and limitations of this transformation (Scheme 25). ${ }^{224}$ Under similar conditions previously reported with this enzyme, ${ }^{223}$ a number of fluoro-, chloro, bromo-, methyl-, methoxy- and dihalogenated compounds gave moderate to very good conversion. Interestingly, for monosubstituted substrates, a general correlation between the conversion and the position of the substituent could be observed: the rate of amination was found to decrease in the order ortho- > meta- > para-. A difference of $>35 \%$ in conversion was observed between the three isomers of fluoro-, chloro- and 
bromo- substrates, for instance. This was ascribed to the steric clash between the substituent and active site residues, as also postulated by the authors. Unsurprisingly, increasing further the size of the aromatic ring either dramatically reduced the conversion values (e.g., 1-naphthyl) or afforded no conversion at all (e.g., indol-3-yl, 3,4-methylenedioxyphenyl). On the other hand, substrates where the phenyl ring was replaced with a short aliphatic chain (e.g., trans-2-butenoic acid) gave no conversion either. Smaller thienylacrylic acids were accepted, albeit with low conversion to the corresponding amino acid. To probe further the substrate tolerance of PAL, $\alpha$ and $\beta$-substituted fluoro- and methylcinamic acid were tested under amination condition. No conversion was observed with these substrates, and they were in fact found to be mildly inhibitory to the enzyme. It is worth mentioning that up to now (more than 25 years later), no wild-type or mutant PAL active on a cinnamic acid derivative bearing any substituent other than $\mathrm{H}$ (or ${ }^{2} \mathrm{H}$ and ${ }^{3} \mathrm{H}$ ) $148,154,225$ on the $\mathrm{C}=\mathrm{C}$ bond has been identified. The same situation applies to the attempts to expand the nucleophile scope: even though deamination of $N$-substituted amino acids has been reported (e.g., $N$-methylphenylalanines ${ }^{226}$ ), amination with methylamine or any other nitrogen nucleophile has not been successful, as of yet. Such extremely challenging targets would represent a major breakthrough and a very valuable addition to the ammonia-lyase toolbox for biocatalysis. 


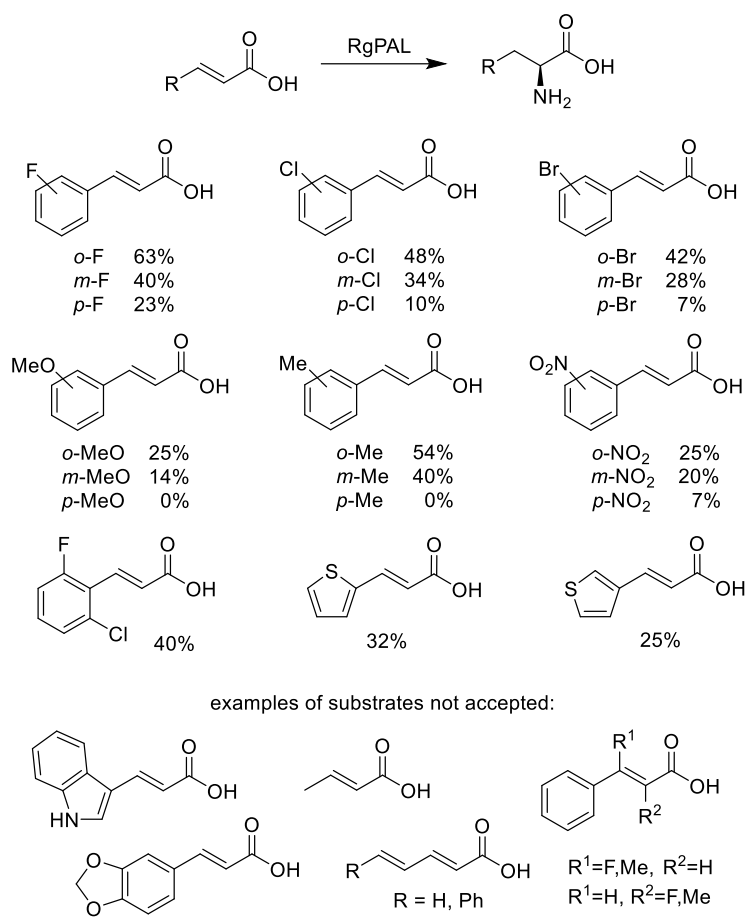

Scheme 25. RgPAL catalyzed synthesis substituted phenylalanine analogues (percentage values refer to conversions).

Bromophenylalanines are particularly valuable products for further chemical manipulations to afford structurally diverse L-phenylalanine analogs (see Section 4.5 for examples of multi-step systems). However, RtPAL and RgPAL suffer from narrow substrate specificity and poor conversions with bromocinnamic acids (particularly the $p$-bromo isomer) limiting their use as industrial biocatalysts. Recent engineering efforts by Rowles et al. successfully improved substrate tolerance of a related PAL (RgrPAL from Rhodotorula graminis) towards $p$ bromocinnamic acid. ${ }^{227}$ The design and screening of five CASTing libraries (each covering two spatially close positions) in the active site allowed the identification of the RgrPAL-His143CysGln144Asn variant (RgrPAL-31E) with an impressive 28-fold improvement in activity compared to the wild-type. Even though AvPAL was found to be more tolerant towards that substrate (71\% conversion), similar engineering work on AvPAL by Ahmed et al. yielded a modest improvement in activity with the single point mutation Phe107Ala ( $80 \%$ conversion). ${ }^{228}$ 
The discovery, cloning and screening of new arylalanine ammonia-lyase enzymes from different sources proceeded at a much faster rate in the last years, occasionally stumbling into remarkable new properties. In 2008, DSM patented the discovery and expression of new PAL genes from several organisms (Shewanella oneidensis, Idiomarina lohiensis, Fibrobacter succinogenes and Vibrio vulnificus) with increased expression levels and very high relative activities on $o$ bromocinnamic acid, compared to RgPAL. ${ }^{229}$ Besides their increased activity, however, the striking feature of this set of new biocatalysts (in particular the PAL from I. lohiensis) was the extraordinary tolerance to high substrate concentrations, even at elevated $\mathrm{pH}$ values where the solubility is higher. The combination of high activity and absence of substrate inhibition is extremely appealing to increase the productivity of industrial biocatalytic processes.

As a further demonstration of the industrial viability of PALs, the intensified synthesis of a number of non-natural halophenylalanine derivatives was reported by Weise et al., in collaboration with the chemical company Johnson Matthey. ${ }^{230}$ Using ammonium carbamate as the ammonia source instead of the more conventional $\mathrm{pH}$-adjusted ammonia solutions or other ammonium salts, considerably improved conversions could be obtained, along with a much simpler downstream processing. The superior conversion was attributed to ammonium carbamate releasing two equivalents of ammonia whilst maintaining a lower ionic strength. Several important parameters including temperature, $\mathrm{pH}$, catalyst formulation, substrate loading and incubation time were optimized to improve the efficiency of the biocatalytic process. Preparative scale synthesis of a number of amino acids was carried out yielding full conversion in each case with a space-time yield (STY) in the range of 5-20 $\mathrm{g} \mathrm{L}^{-1} \mathrm{~d}^{-1}$ (Scheme 26). The use of ammonium carbamate improved the isolation efficiency by evaporation of the buffer at its sublimation temperature, alleviating many problems encountered using previous methods, such as the requirement for ion-exchange purification (with additional costs and sometimes low recovery). Optimized conditions for 3-fluorocinnamic acid led to an impressive STY of $237.6 \mathrm{~g} \mathrm{~L}^{-1} \mathrm{~d}^{-1}$ with excellent enantiopurity. To demonstrate the modularity of this process, the method was also 
integrated in two chemo-enzymatic processes (synthesis of biarylalanine derivatives ${ }^{228}$ and telescopic one-pot condensation/hydroamination, ${ }^{231}$ see Section 4.4).

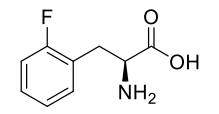

$18.3 \mathrm{gL}^{-1} \mathrm{~d}^{-1}$

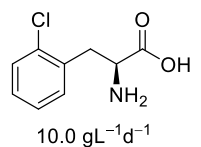

$10.0 \mathrm{gL}^{-1} \mathrm{~d}^{-1}$

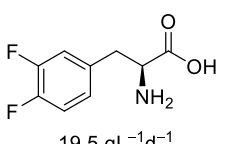

$19.5 \mathrm{gL}^{-1} \mathrm{~d}^{-1}$

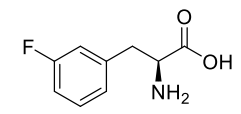

$237.6 \mathrm{gL}^{-1} \mathrm{~d}^{-1}$
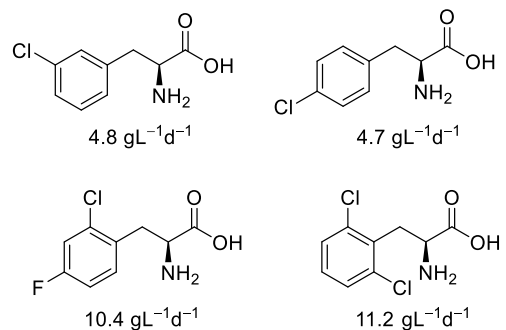

Scheme 26. Panel of non-natural L-halophenylalanines produced with an intensified AvPAL mediated hydroamination using ammonium carbamate as the ammonia source (space-time yields are reported).

Previous work has alluded to the fact that no modification of the carboxylic acid portion of the substrate is tolerated by the enzyme. Remarkably, Nair and co-workers reported the PAL catalyzed hydroamination of methyl trans-cinnamate to L-phenylalanine methyl ester using RgPAL in an organic-aqueous biphasic mixture, due to the poor solubility of the substrate (Scheme 27). ${ }^{232}$ A solvent screen showed $n$-heptane to be the best co-solvent, giving the highest product yield. Under the best conditions, with a substrate loading of $100 \mathrm{mM}$, only $7 \%$ conversion could be achieved. While the potential applications (e.g., for aspartame synthesis) are exciting, the low conversions obtained from the biotransformation are far from ideal for industrial application. Unfortunately, no further work through reaction optimization or enzyme engineering was reported with this substrate, which suggests the extreme difficulty in engineering PAL enzymes to accept substrates lacking the carboxylate moiety essential for substrate binding. 


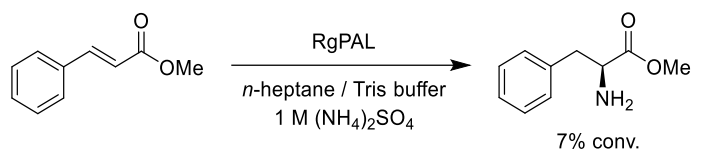

Scheme 27. RgPAL catalyzed synthesis of L-phenylalanine methyl ester in a biphasic system.

Several phenylalanine analogues with non-aromatic cyclic systems were explored by different groups, either for mechanistic investigation or in the attempt to understand and expand the substrate spectrum. Ressler et al. demonstrated promising activity towards the deamination of L(2,5-dihydrophenyl)alanine, to afford the corresponding acrylic acid with RgPAL. Kinetic data revealed 10 -fold decrease in $k_{c a t}$ but similar binding affinity to L-phenylalanine, yielding $85 \%$ conversion after 4 days (Scheme 28a), ${ }^{233}$ disputing earlier works suggesting that PAL enzymes do not accept non-aromatic substrates. A tentative explanation for the deamination was provided two decades later by Retéy and co-workers using the Friedel-Crafts mechanism. ${ }^{234}$ They also postulated the regioisomer L-(1,4-dihydrophenyl)alanine to be a competitive inhibitor of PAL, and this was proved through inhibition studies with PcPAL (Scheme 28a). Gloge et al., in pursuit of unique substrates for potential novel applications, tried unsuccessfully the hydroamination of cyclooctatetraenylacrylic acid (Scheme 28b) ${ }^{235}$ The lack of activity can be attributed to three key factors: the non-aromatic character, the size and the conformation of the ring. Earlier work on PALs highlighted the requirement of substrates to bear a planar 6-membered aromatic ring for optimal substrate binding and catalysis. The increased size of the 8-membered ring could lead to a clash with the active site residues lining the hydrophobic pocket, although the thermodynamically favored puckered conformation adopted by the non-aromatic ring might be the key factor that prevents the substrate from binding effectively in the active site. 
(a)
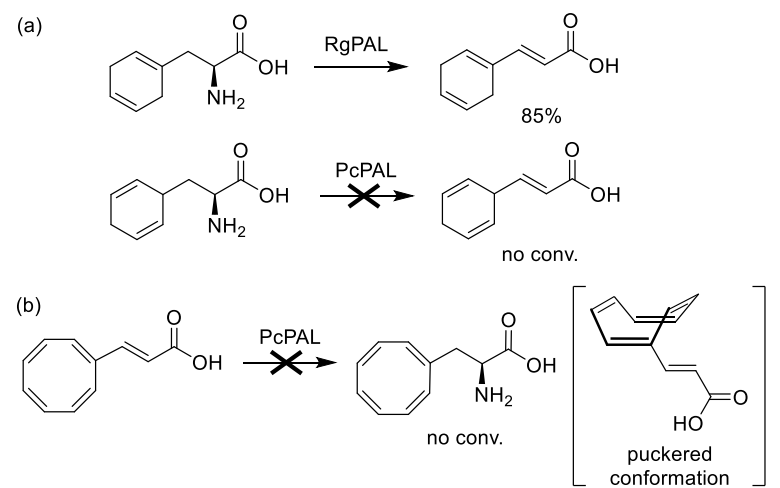

(c)
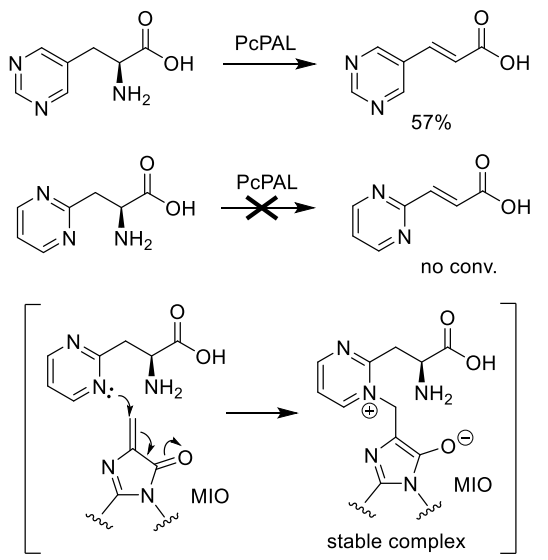

Scheme 28. Early studies of amination/deamination of non-conventional PAL substrates: dihydrophenylalanine isomers (a), cyclooctatetraenylacrylic acid (b), pyrimidinylalanines (c).

Additional mechanistic work on PcPAL was carried out testing the deamination of two aromatic pyrimidine substrate analogues: L-(pyrimidin-2-yl)alanine and L-(pyrimidin-5-yl)alanine (Scheme 28c). ${ }^{151}$ The pyrimidin-2-yl regioisomer was determined to be a competitive inhibitor, forming a strong irreversible covalent bond with the MIO functionality leading to a stable pyrimidinium complex. This was interpreted as possible supporting evidence for the FriedelCrafts-type mechanism, even though the formation of this inert complex does not in itself disprove the E1cB mechanism. On the other hand, the pyrimidin-5-yl isomer was accepted by PcPAL to give the acrylic acid with a respectable $57 \%$ yield.

Nonetheless, these fundamental studies led also to the exploration of the substrate scope of PcPAL, highlighting its broad substrate tolerance and its industrial value in biocatalytic synthesis 
of non-natural amino acids, mainly through the work of Rétey, Poppe and co-workers. Several polyfluoro- and monochloro- phenylalanine analogues were synthesized with moderate to good isolated yields (37-88\%) with high enantiopurity using purified enzyme (Scheme 29). Nitrogencontaining heteroaromatic substrates were also tested, affording excellent conversion for all three regioisomers of pyridylacrylic acid and (pyrimidin-5-yl)acrylic acid. The substrate scope of PcPAL was then probed with furan and thiophene systems, including larger fused aromatic rings (Scheme 29). ${ }^{236}$ Many of these substrates were converted efficiently with good isolated yields, even though some were previously shown to give poor or no activity with fungal PALs. ${ }^{224}$ PcPAL failed to convert only the substrates containing 3-substituted furans and thiophenes. The lack of activity towards these substrates was ascribed to the unfavorable binding conformation the substrates adopted in the active site, seen in the light of the Friedel-Crafts-like mechanism. ${ }^{144}$ However, the 3-thienylacrylic acid substrate is accepted to some extent by RAPL, ${ }^{224}$ pointing towards either a different mechanism or an altered substrate binding mode in a different enzyme. The substrate tolerance of PcPAL was further challenged with 5-arylfuran-2-yl derivatives, affording the corresponding biarylalanines in 49-66\% isolated yields after 36-48 $\mathrm{h}$ (Scheme 29). ${ }^{237}$ Nitro-substituted phenylalanines were also obtained in very good yields. ${ }^{139,153}$ Access to such a broad variety of compounds through one simple biocatalytic method (with excellent enantiomeric purity in all cases) opened up potential sustainable routes to the manufacture of countless non-natural amino acid derivatives for the pharmaceutical and agrochemical industry. Furthermore, such a broad substrate scope facilitated the development of PAL-specific assay methods using modified or functionalized cinnamic acid analogues as molecular probes. ${ }^{238,239}$ 


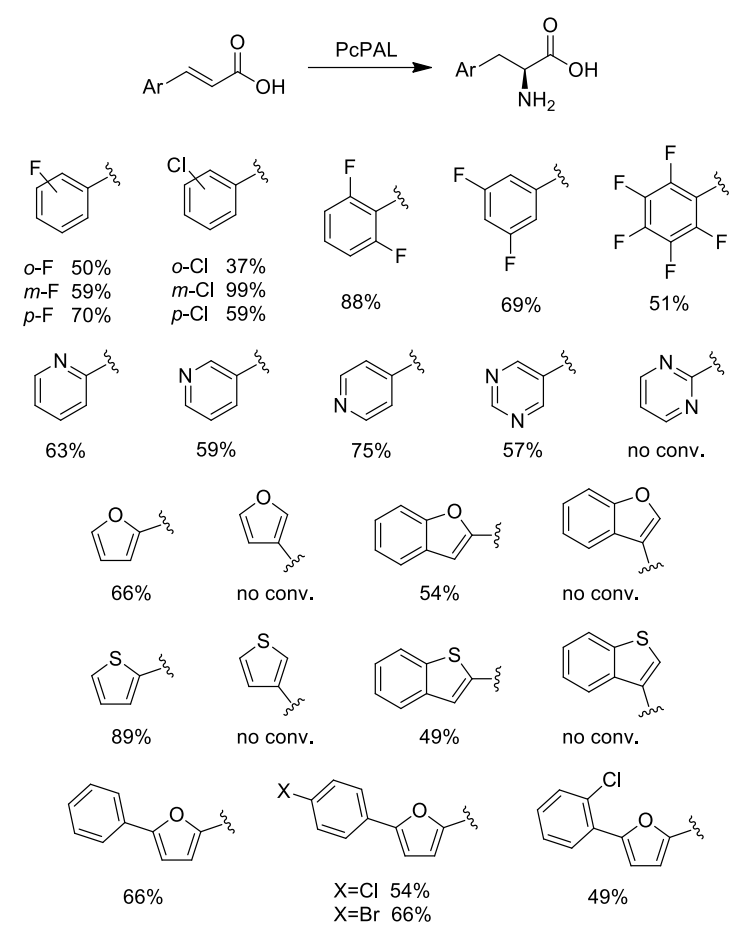

Scheme 29. Production of a broad panel of aromatic and heteroaromatic amino acids by PcPAL (percentage values refer to isolated yields).

The discovery of the cyanobacterial AvPAL from Anabaena variabilis ${ }^{112}$ led to an expansion of the substrate scope of PAL aminations for the production of non-natural amino acids. When comparing its catalytic activity with that of PALs from yeasts (RgPAL) and plants (PcPAL), AvPAL presented an even broader substrate scope towards several non-natural substrates. ${ }^{240}$ This was highlighted with the synthesis of L-(4-trifluoromethyl)phenylalanine (a substrate not accepted by RgPAL and PcPAL) on a preparative scale, with excellent conversion and enantiopurity, in a moderate isolated yield of $42 \%$ (Scheme 30).

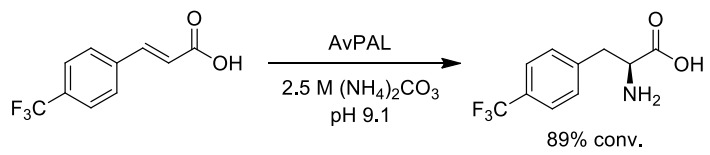

Scheme 30. Preparative scale synthesis of L-(4-trifluoromethyl)phenylalanine with AvPAL. 
As a further illustration of the synthetic utility and the robust activity of PAL enzymes, Barron et al. investigated the synthesis of L-phenylalanines by RgPAL in ionic liquids (ILs). Four commonly used ILs were tested: 1-butyl-3-methylimidazolium methylsulfate ([BMIM][MeSO 4$]$ ), 1-butyl-3-methylimidazolium tetrafluoroborate ([BMIM][BF $]$ ), 1-butyl-3-methylimidazolium hexafluorophosphate ([BMIM] $\left.\left[\mathrm{PF}_{6}\right]\right)$ and 1-butyl-3-methylimidazolium lactate ([BMIM][lactate]). Ionic liquids tend to infer thermal/operational stability and generally show reduced enzyme inhibition/deactivation compared to organic solvents. [BMIM] $\left[\mathrm{PF}_{6}\right]$ was found to be the best IL for this application, giving a maximum conversion of $59 \%$ in the amination direction using $1 \mathrm{M}$ ammonium hydroxide adjusted to $\mathrm{pH} 9.0 .^{241}$

Enzyme immobilization, a widely used method to improve the lifespan and operational stability of enzymes for industrial applications, has been recently demonstrated with PALs. The ability to recycle enzymes after each biotransformation is an attractive strategy to improve overall efficiency and to reduce waste and costs. The advent of microreactor technology has rekindled efforts in developing innovative processes with immobilized enzymes. Pioneering work conducted by Poppe and co-workers demonstrated the potential application of immobilized arylalanine ammonia-lyases. ${ }^{242}$ PcPAL was covalently attached to carboxylated single-walled carbon nanotubes (SwCNT), through either a direct link or a glycerol diglycidyl ether (GDE) linker (Scheme 31). To determine the stability of the covalently immobilized enzyme, optimization tests were carried out with 2-thienylacrylic acid as a model substrate, under harsh amination conditions $\left(6 \mathrm{M} \mathrm{NH}_{4} \mathrm{OH}, \mathrm{pH} 10.0\right)$. The directly linked enzyme suffered significant loss of activity after three cycles, while the GDE linked enzyme showed greater tolerance, retaining $98 \%$ activity after three cycles and $50 \%$ after five cycles. Optimal amination conditions in continuous flow mode were found to be $2 \mathrm{M} \mathrm{NH}_{4} \mathrm{OH}, \mathrm{pH}$ 10.0. The difference in conversion between immobilized and free enzyme (58\% compared to $89 \%$ ) is due to the low ammonia concentration, in order to prevent hydrolysis and degradation of the covalent linker. Immobilized 
PcPAL showed remarkable thermal stability compared to the free enzyme: no significant loss of activity at $70^{\circ} \mathrm{C}$ for $38 \mathrm{~h}$, with an optimum temperature found to be around $60^{\circ} \mathrm{C}(1.5$-fold increase in temperature tolerance compared to the free enzyme). This method positively affects two key parameters of the biocatalyst, its reusability over several cycles and the its thermal tolerance, both important for industrial manufacturing. A very recent report from the same group demonstrated even higher stability and durability of PcPAL immobilized on aminated SwCNTs compared to carboxylated SwCNTs ${ }^{243}$ Experiments aimed at demonstrating the recyclability of the immobilized biocatalyst highlighted an unusual inactivation profile, with a relatively long stable plateau, followed by rapid inactivation. This led to the interesting hypothesis that the deactivation is caused by the slow (and reversible) replacement of structural water with ammonia, leading to a sudden loss in activity only when the inner regions close to the active site are affected. This hypothesis seems to be confirmed by experimental results, since the original activity could be restored multiple times by incubating the deactivated immobilized biocatalyst with a slightly acidic reconditioning buffer after each run. ${ }^{243}$

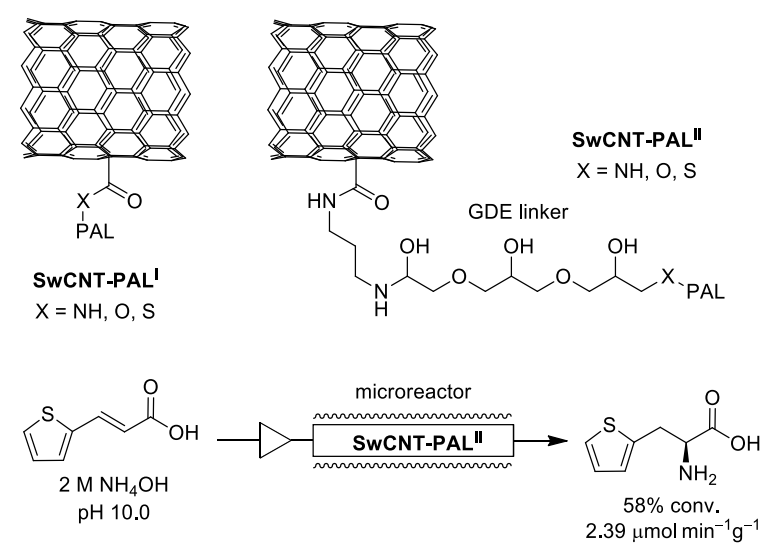

Scheme 31. Immobilization of PcPAL on a carboxylated single-walled carbon nanotube (SwCNT) and application to the synthesis of L-arylalanines using microreactor technology. 
Additional work carried out by the same group demonstrated the immobilization of PcPAL on magnetic nanoparticles (MNPs). ${ }^{149}$ The MNPs-immobilized enzyme was retained by means of permanent magnets in several small cells within a microfluidic reactor. Remarkably, the enzyme in the microfluidic system managed to carry out successfully the synthesis of L-propargylglycine from the corresponding acrylic acid, a substrate which was thought to be incompatible with PAL enzymes, affording 14\% isolated yield (Scheme 32). This contradicts earlier works which suggested the substrates require an aromatic ring to enable binding to the active site, and also disfavors the Friedel-Crafts mechanism. More importantly, Poppe and co-workers have highlighted new opportunities towards catalyzing the conversion of acyclic acrylic acid analogues, broadening the substrate scope and potential applications of ammonia-lyases.

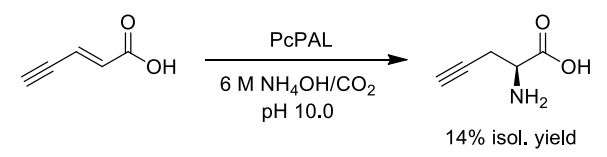

Scheme 32. Synthesis of L-propargylglycine using PcPAL (on MNPs) in a microfluidic system.

\subsection{Synthesis of D-arylalanines}

The biocatalytic synthesis of the non-proteinogenic enantiomer of amino acids represents an attractive strategy in industrial processes compared to conventional chemical synthesis. Where direct asymmetric synthesis is not possible, two main approaches exist to produce optically pure D-arylalanines: kinetic resolution (stereoselective removal of the L-enantiomer) or deracemization (two-step conversion of the L-enantiomer to either the D-enantiomer or the racemic mixture, through a non-chiral intermediate). Both processes have typical advantages and disadvantages. For example, while kinetic resolution might be a much simpler and cheaper process, the maximum conversion is limited to $50 \%$, making the process intrinsically wasteful. Employing a deracemization cascade can mitigate this problem by recycling the L-enantiomer, 
with theoretical conversion $>99 \%$, although the process is usually more complex, requiring several components for the reaction. PALs have been used for a long time in the synthesis of Darylalanines by stereoselective deamination of the L-enantiomer in a chemically synthesized racemic mixture. The acrylic acid can be removed easily (e.g., by ion-exchange chromatography or recrystallization), leaving behind the unconverted D-product. An additional advantage of this method is that the PAL reaction (in the natural direction) proceeds more efficiently without the need for high $\mathrm{pH}$ and ammonia concentrations. PAL-based deracemization approaches were developed only very recently.

An early study reported the synthesis of a range of D-arylalanines with $>99 \%$ ee and moderate to good isolated yields using a crude homogenate of Rhodotorula graminis cells. ${ }^{244}$ Even though the method may be considered rudimental since the lysate contains a number of uncharacterized enzymes (including, most likely, RgrPAL), it highlights the simplicity and low cost of the process, which led to the resolution and isolation of several substituted phenylalanine analogs (Scheme 33).

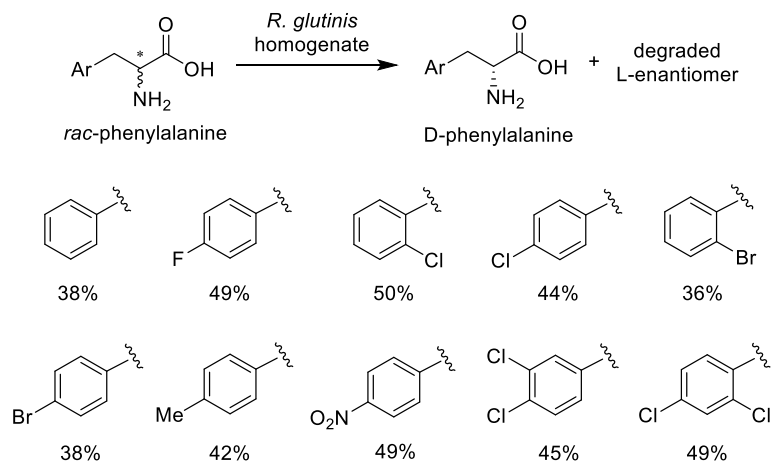

Scheme 33. Isolation of enantiopure D-phenylalanines from racemic mixtures using Rhodotorula graminis homogenate (percentage values refer to isolated yields).

A similar strategy was also reported by Shibatani et al. exploiting the HAL activity of Achromobacter liquidum cells, for the production of urocanic acid and D-histidine. ${ }^{245}$ The 
conversion rates were improved by addition of triethanolamine lauryl sulfate (TEALS), a surfactant to increase the permeability of the membrane. No inhibition of HAL was observed in the presence of TEALS and $45 \%$ isolated yield of D-histidine and $46 \%$ isolated yield of urocanic acid was obtained from racemic histidine (Scheme 34).

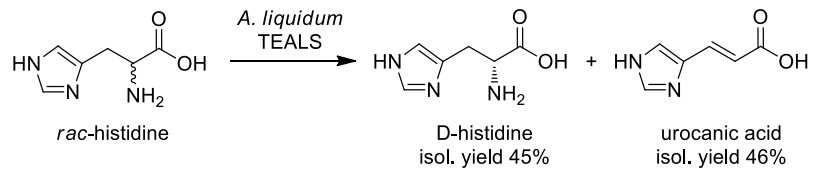

Scheme 34. Synthesis of D-histidine and urocanic acid from the enantioselective deamination of rac-histidine.

Rétey and co-workers ${ }^{153,236,237}$ and later Poppe and co-workers ${ }^{149,242}$ employed isolated PcPAL for the production of a very broad panel of enantiomerically pure D-heteroarylalanines via the consumption of the L-enantiomer (Scheme 35). Furthermore, Zhu et al. demonstrated the large scale resolution of racemic phenylalanine in a packed-bed reactor using covalently immobilized RgPAL on a mesoporous silica support, to give $>99 \%$ conversion with a space-time yield of $7.2 \mathrm{~g}$ $\mathrm{L}^{-1} \mathrm{~h}^{-1}$ and perfect enantioselectivity. ${ }^{246}$ 


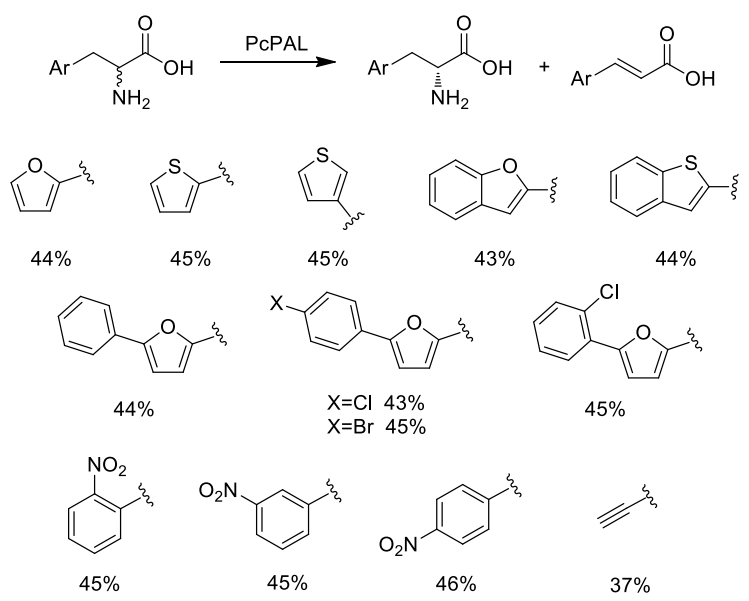

Scheme 35. Isolation of enantiopure D-arylalanines from racemic mixtures using PcPAL (percentage values refer to isolated yields).

To overcome the $50 \%$ theoretical yield limitation of kinetic resolution approaches, Parmeggiani et al. recently proposed a chemo-enzymatic amination-deracemization strategy for the synthesis of D-phenylalanines directly from cinnamic acids (Scheme 36). ${ }^{155}$ Firstly, a PAL converts the cinnamic acid into L-phenylalanine (enantiopure or containing small amounts of D-enantiomer, depending on substituents), then the L-amino acid deaminase from Proteus mirabilis (LAAD) selectively oxidizes the L-enantiomer, rapidly followed by its non-selective chemical reduction by ammonia-borane to afford rac-phenylalanine. Repeated cycles of L-selective oxidation and non-selective reduction result in accumulation of the D-enantiomer in very high ee. Solid-phase screening of a very large site-saturation mutagenesis library of AvPAL afforded variants with improved activity towards D-phenylalanine production (AvPAL-His359Tyr found to be the most efficient). Even though the PAL variants did not afford enrichment in the D-enantiomer, they allowed the racemic composition to be reached more quickly, and performed better in the cascade system. This procedure allowed the production of enantiopure D-amino acids with $>98 \%$ ee and conversions between $62-80 \%$. A similar system, employing a D-amino acid oxidase (DAAO) for selective removal of D-product in place of the LAAD, allowed to improve to $>99 \%$ ee the optical 
purity of those L-arylalanines that were not obtained in enantiopure form with PAL mediated aminations. $^{155,247}$
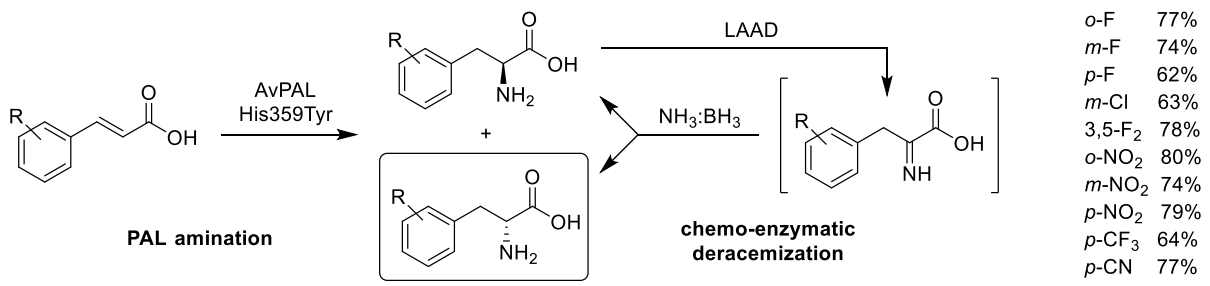

Scheme 36. Chemo-enzymatic amination-deracemization cascade for the synthesis of Dphenylalanines from cinnamic acids (percentage values refer to isolated yields).

\subsection{Synthesis of $\beta$-arylalanines}

$\beta$-amino acids have been described as an emerging class of synthons for a number of years ${ }^{163,248,249}$ and find use particularly in the area of medicinal chemistry. As they are isomers of the proteinogenic amino acids, they are especially useful as building blocks for therapeutic polymers mimicking peptide-peptide interactions, which display promising bioavailability and in vivo stability when compared to natural proteins. ${ }^{250-252}$ Derivatives of these chemicals with aromatic side chains are also used in the synthesis of small molecule pharmaceuticals, as well as being found in bioactive natural products (Scheme 37). Although the use of $\beta$-amino acids seems to be less widespread than that of the $\alpha$-isomers, it is unclear whether this is due to lack of focus on asymmetric methods to provide amenable routes to these compounds. 

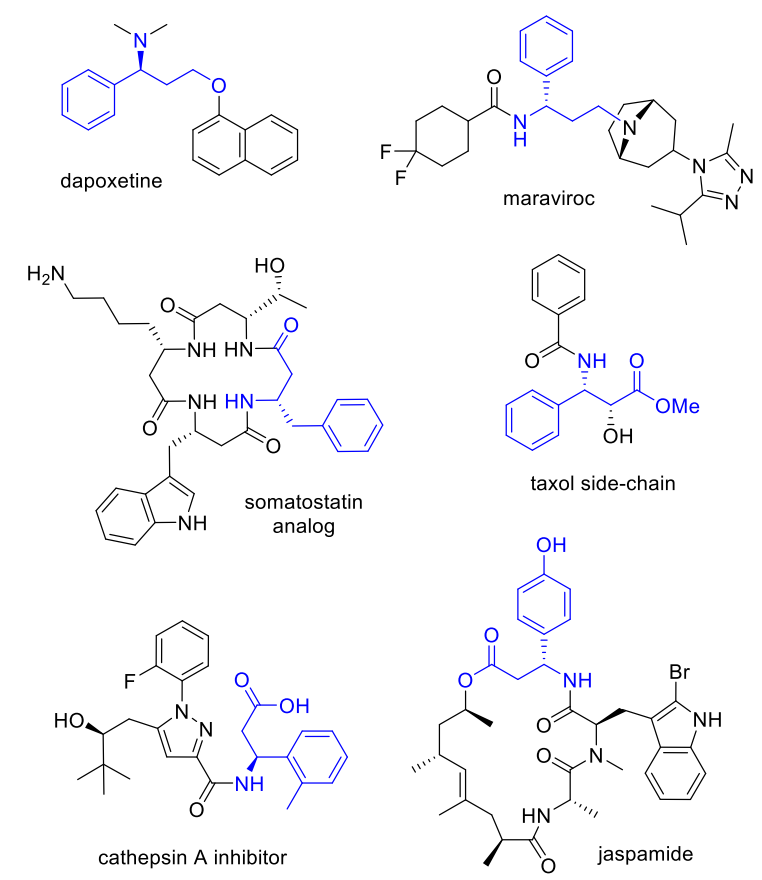

Scheme 37. Representative examples of bioactive compounds synthesized from $\beta$-aromatic amino acids (the arylalanine-derived moieties are highlighted in blue).

In contrast to all the other aminomutase classes, the MIO-containing aminomutases have great potential as industrial biocatalysts due to their ability to perform amino acid isomerization chemistry in a unique way, without the need for expensive cofactor supplementation. As the reaction involves interchange between the $L-\alpha$ - and either enantiomer of the $\beta$-amino acid via the corresponding acrylic acid, there are three main methods by which optically pure $\beta$-arylalanines can be produced using this class of enzymes. The first of these is via isomerization of Lphenylalanine or its derivatives using either an $(R)$ - or $(S)$-PAM with high enantioselectivity, providing complementary routes to either enantiomer (Scheme 38, center). These reactions can also be performed in reverse using racemic starting material to yield enantiopure products via kinetic resolution (Scheme 38, left side). Lastly, the intermediate of the wild-type reaction can be used as a starting material for direct addition of ammonia, in a similar manner to the use of PALs as hydroamination biocatalysts (Scheme 38 , right side). 


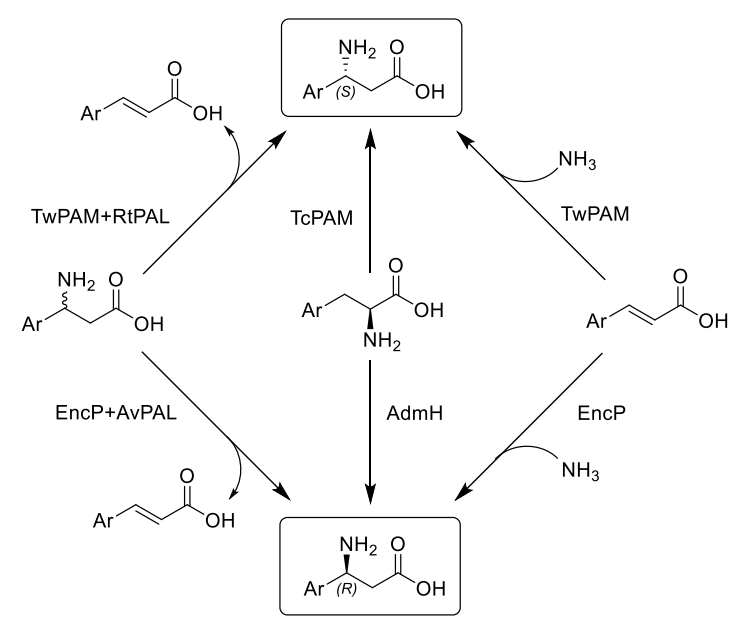

Scheme 38. Examples of biocatalytic strategies for the production of $(R)$ - or $(S)$ - $\beta$-arylalanines employing enzymes with enantiocomplementary aminomutase activity.

Among the proteins with aminomutase activity in the class I lyase-like family, tyrosinemetabolising enzymes have received much less attention than their phenylalanine-specific counterparts for use in the biocatalytic production of $\beta$-arylalanines. One reason for this is that many of the characterized TAMs from bacteria have been observed to possess low enantioselectivity with respect to the $\beta$-tyrosine product ${ }^{134,157,163}$ when compared to known PAMs, which do not accept para-hydroxylated substrates. ${ }^{175,177,187}$ This feature of TAMs, studied more extensively in the $(R)$-selective $\mathrm{CmdF}$ from Chondromyces crocatus, has proved difficult to explain fully and thus remedy via site-directed mutagenesis. ${ }^{134}$ In spite of this drawback, the ability to generate synthetically valuable $\beta$-amino acids with excellent optical purity from their cheaper and more readily available proteinogenic regioisomers remains attractive from a commercial point of view. As such, efforts have been made to design enzymes with improved industrial characteristics, for instance enantioselectivity, to allow $\beta$-tyrosine to be accessed as a chiral building block. This has been achieved through the rational engineering of the $(R)$-selective TwPAM from Taxus wallichiana, making use of sequence alignments between this enzyme and 
its relatives with TAM activity. Identification of variation around the aryl binding pocket within a subset of family members allowed amino acid exchanges to be introduced with the hope of conferring TAM-like substrate specificity to the template enzyme, whilst retaining its excellent native enantioselectivity. The most successful of these was TwPAM-Cys107Ser, which was shown to enable both rearrangement of L-tyrosine and amination of $p$-coumarate to give the corresponding (R)- $\beta$-amino acid without any trace of the unwanted $(S$ )-enantiomer (or $\alpha$ regioisomer in the latter case). ${ }^{253}$ More recently the eukaryotic $(R)$-selective OsTAM1 has been discovered in the rice plant Oryza sativa ${ }^{165}$ which may be a more amenable candidate for fine chemical production. This enzyme has been shown to be extremely selective for tyrosine $v s$. phenylalanine (unlike TwPAM-Cys107Ser) ${ }^{253}$ whilst possessing enantioselectivity superior to that of any other wild-type TAM. ${ }^{166}$ Interestingly, OsTAM1 has been shown to have an active site very similar (two amino acid differences) to plant PALs ${ }^{167}$ and thus TwPAM ${ }^{180}$ with variant residue Tyr125 at the position homologous to the Cys107. As a Cys107Tyr exchange was not attempted in the aforementioned rational design work, this reinforces not only that there are hotspot positions for activity in the aryl binding pocket of PAM/PAL/TAM enzymes, ${ }^{167}$ but also that the variations selected initially in TwPAM may not have arrived at the optimal residueresidue combination. Continued enzyme discovery and engineering efforts in this understudied area could see growth in the use of TAMs as commercially-viable biocatalysts in the future (Scheme 39). 

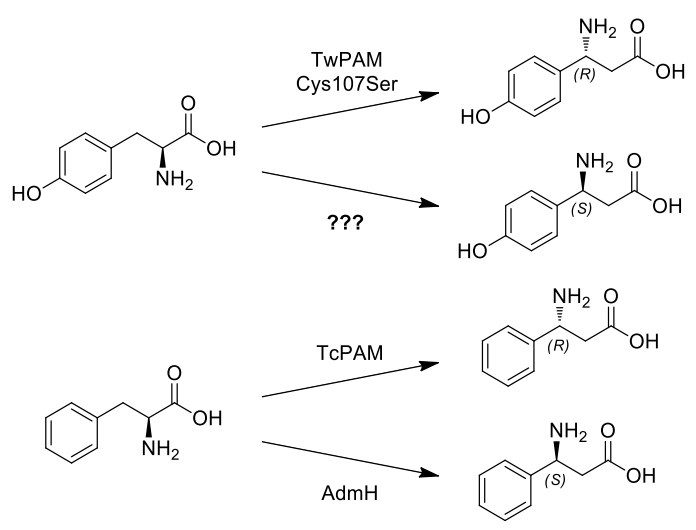

Scheme 39. Possible and reported biocatalytic strategies to produce enantiopure $\beta$-arylalanines from the renewably sourced primary metabolites L-tyrosine and L-phenylalanine.

Aside from the engineering of PAMs to perform the TAM reaction, the wild-type substrate scope of the isomerization reaction has been of great interest to researchers in the field of biocatalysis. As PAMs do not seem to have a requirement for a para-hydroxy functionality in their substrates, the range of compounds which can be accepted is broader and thus their use is more widely applicable. Nearly 10 years after the initial identification of PAM activity in the Taxus genus, ${ }^{174}$ the use of TcPAM for the conversion of various L-arylalanine starting materials, including Lphenylalanine, to the $(R)-\beta$-isomers was reported. The enzyme was found to accept a range of ring substituents, including fluorine and methyl groups at any position and methoxy and tert-butyl groups at the para-position, as well as the more unusual 2-furyl-, 2-thienyl- and $\beta$-styryl-Lalanines. ${ }^{254}$ Although a value-added process, the requirement for enantiopure starting materials could be seen as a drawback in an industrial setting, a concern addressed by the coupling of TwPAM with a broad specificity alanine racemase from Pseudomonas putida. ${ }^{255}$ This method, subsequently patented by Michigan State University, ${ }^{256}$ allowed dynamic kinetic resolution of more readily accessible racemic $\alpha$-amino acids, resulting in an improvement in yield of up to $19 \% .{ }^{255,256}$ With later structural insight into the ligand binding interactions of TwPAM, it was possible to rationalize and enhance the poorer acceptance of some L-amino acid substrates. With 
the example of L-3-methylphenylalanine, the ring substituent was predicted to clash with residue Leu104 in the aryl binding pocket in a model based on the structure of the TcPAM-cinnamate complex. Through rational design, the TcPAM-Leu104Ala variant was created and shown to give more favourable kinetic parameters with the substrates under study, although not with the 4methylphenylalanine or styrylalanine analogues. ${ }^{170}$ The acceptance of $(S)-\beta$-styrylalanine by the enzyme was an interesting finding in that, unlike most other substrates, the enzyme was found to produce almost exclusively styrylacrylic acid, the intermediate of the aminomutase reaction. The lack of reamination in this case was seen to imply that the abstracted amine was retained in the active site for longer than the substrate, ${ }^{257}$ which was later confirmed by burst phase analysis of the reaction, with rate constants revealing temporary inactivation (covalent amine-MIO adduct) and reactivation (ammonia release/reamination) of the enzyme. ${ }^{258}$ This feature of the reaction with styrylalanine and bicyclic alanine derivatives was shown to allow intermolecular amine transfer, thus enabling the introduction of much cheaper, non-chiral arylacrylic acids as starting materials ${ }^{257}$ in place of the perhaps less industrially desirable enantiopure amino acid substrates (Scheme 40).

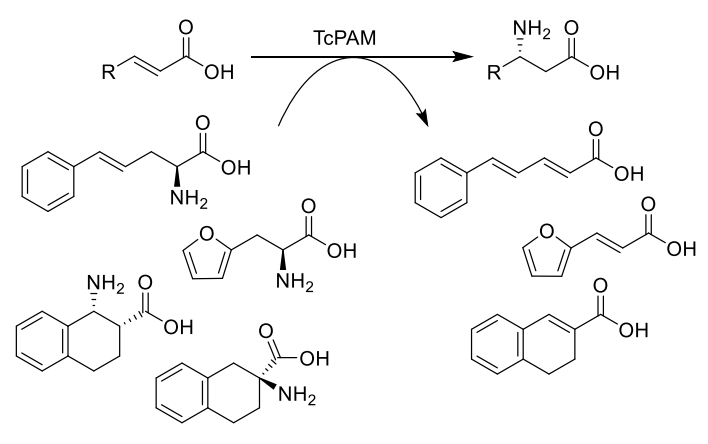

Scheme 40. Examples of biocatalytic ammonia transfer reactions as catalyzed by the $(R)$-selective TcPAM. 
At around the same time as the initial work with TcPAM was being reported, the Japanese company Mitsui Chemicals Ltd. filed patents detailing the use of (S)-PAMs (specifically AdmH) for the production of $(S)$ - $\beta$-phenylalanine via isomerization. ${ }^{259,260}$ This method employed whole cells, rather than the more intensive and costly isolated enzyme formulation common in other research, with $94 \%$ conversion of $90 \mathrm{~g}$ starting material and $>99 \%$ enantiomeric excess values in the best case example. ${ }^{260}$ This large scale and efficient overall biotransformation is reported to be enabled by specific conditions, with temperature, $\mathrm{pH}$ and buffer composition being optimized for precipitation of the product, thus overcoming the equilibrium limitation of the mutase reaction. ${ }^{260}$ Similar results have also been achieved with a wider range of compounds by employing the whole cell biocatalyst within a fermentation process. By adding different non-natural L-amino acids to the growth medium of an AdmH production culture, it was possible to see conversions of over $90 \%$ to several (S)- $\beta$-arylalanine derivatives (3-methylphenyl-, 3- and 4-bromophenyl-, 4chlorophenyl- and 2-thienyl-). ${ }^{249}$ This route was able to afford $235 \mathrm{mg}$ product per litre of culture with the highest converted substrate, although at this concentration the precipitation of the $\beta$ amino acid during production was not reported $^{249}$ as with the previous, more intensified method. ${ }^{260}$ As an alternative explanation of the displaced equilibrium between the freely interchanging $\beta$ - and $\alpha$-amino acids, the authors suggest differential permeation of the bacterial cell walls by the regioisomers during the growth phase of the culture. In this way, they predict a compartmentalization effect leading to accumulation of enantiopure $\beta$-arylalanine in the extracellular environment with $\alpha$-amino acid permeases contributing to a form of in situ substrate feeding. ${ }^{249}$ Additionally, the whole cells were found to be reusable for at least five reaction batches without any decrease in activity, which is an attractive quality in an industrial catalyst and possibly due to the implementation of the biotransformation in growth medium, at low temperature and over a short duration.

Aside from these industrial and preparative strategies, further academic investigations have been carried out into the use of the AdmH mutase reaction with a selection of chemical probes. One 
such study in 2014 tested a total of 22 L-arylalanine substrates and revealed different reaction rates and product distributions for each. Whilst EWGs at the ortho-position gave no discernible traces of either possible product, their presence at other positions gave mostly $\beta$-amino acid, whereas EDGs gave increased amounts of acrylic acid. These findings could be rationalized, in part, by electronic and resonant effects pertaining to the ease of proton abstraction in the initial deamination step and reamination at the $\beta$-position of the arylacrylate intermediate. ${ }^{248}$ Docking of the more anomalous 3 -substituted cinnamate derivatives in the active site of the enzyme revealed several residues which may influence the substrate positioning and effect the product ratios. ${ }^{248}$ Interestingly one of these, Val108, is homologous to position Val83 in a related enzyme with similar (S)-PAM activity (EncP from Streptomyces maritimus). Variation of this residue to an alanine in EncP is known to increase 20 -fold the rate of cinnamate production from Lphenylalanine. ${ }^{192}$ This may be through the influence of this residue on the mutase/lyase ratio of (S)-PAMs, with clashes from ligand substituents having a similar effect by overlapping with this position in the wild-type active site.

These findings with AdmH have been expanded with further substrate screens of the enzyme, this time in both directions (starting with either L-/rac- $\alpha$-arylalanines or $(S)$-/rac- $\beta$-arylalanines). Apart from slight variations, possibly due to different reaction conditions, the results for $\alpha$ to $\beta$ isomerisation correlate well between the two studies. ${ }^{248,261}$ Again, modelling was used to provide insight into the reaction parameters, but this time comparing the lowest energy states of both $\alpha$ and $\beta$-regioisomers of identically substituted amino acids. These analyses corroborated previous evidence that most meta-substituents preferentially bind in the $\mathrm{NH}_{2}$-cis conformation ${ }^{248,261}$ and revealed additionally that ortho-substituents, as seen in the case with 2-chloro-, may bind best in opposing conformations depending on whether the amine is at the $\alpha$ - or $\beta$-position. ${ }^{261}$ Experiments performed using $\beta$-arylalanines as starting materials also revealed additional information about the AdmH mutase reaction. Whereas 2-nitro-, 2-chloro- and 2-bromosubstituted $\alpha$-phenylalanines were not seen to react in the forward direction, ${ }^{248,261}$ a significant 
amount of $\alpha$-amino acid could be produced when starting with the corresponding $\beta$-isomer. ${ }^{261}$ This provides evidence that the $\alpha$-forms of these amino acids do bind in the active site of the enzyme and take part in the reaction, possibly even being deaminated and reaminated at the same position due to electronic and steric considerations. These structural and mechanistic insights into the chemistry and substrate profile of the industrially exploited (S)-PAM AdmH provide much scope for further application and engineering of this biocatalyst for use in the synthesis of highvalue non-natural amino acid products.

As demonstrated, the aminomutase reaction is freely reversible, meaning that enzymes of this class can also be employed in the kinetic resolution of racemic $\beta$-amino acids, leaving behind the more valuable single enantiomers in high optical purity. AdmH has been recently investigated for this purpose as part of the wider study into the influence of various ring substituted substrates on the mutase reaction mentioned previously. ${ }^{261}$ Using various rac- $\beta$-arylalanines, good acceptance of a variety of substrates, particularly those with ortho-substituents, could be seen (Scheme 41a), as well as evidence that the unreacting $(R)$-enantiomer does not act as a significant inhibitor of the enzyme. However, the reaction was found to result in varying amounts of corresponding cinnamic acid derivative and enantiopure L-amino acid as by-products depending on the substrate, ${ }^{261}$ a feature that may well prove incompatible with industrial processes, where minimal side-products formation and simple purification techniques are often preferred. This consideration had been previously addressed via the design of an earlier kinetic resolution strategy consisting of a biocatalytic cascade combination of TwPAM-Gln319Met ${ }^{178}$ with RtPAL (Scheme 41b). In an elegant manner, any $\alpha$-amino acid produced by the $(R)$-selective aminomutase could be converted immediately to the same arylacrylic acid that may derive from the transformation catalyzed by the TwPAM variant, enabling simple removal of this contaminant via acidification and ion exchange. ${ }^{262}$ In an attempt to combine the enantiopreference of the AdmH work with the preparative scale cascade method, we developed a complementary strategy, combining the $(S)$ PAM activity of EncP from Streptomyces maritimus with AvPAL from Anabaena variabilis 
(Scheme 41c). ${ }^{189}$ This cascade was optimised for the biotransformation of a particular target substrate (rac-3-fluoro- $\beta$-phenylalanine), combined with the chemical synthesis of the starting material in a telescopic manner and subsequently performed at $100 \mathrm{mM}$ scale to give the $(R)$ product in $98 \%$ ee.
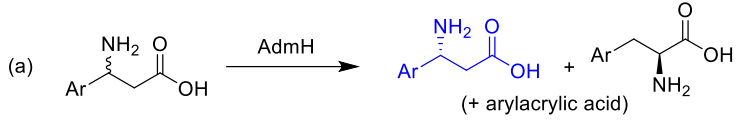

(b)
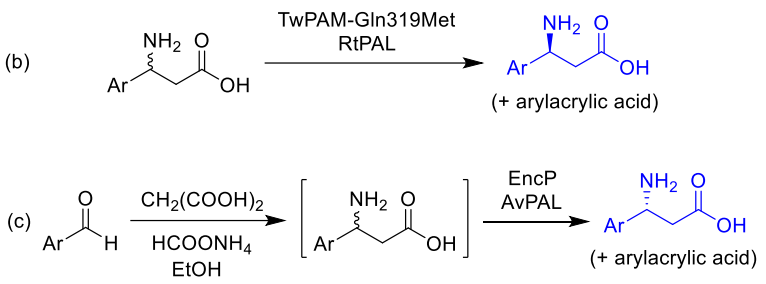

Scheme 41. Examples of kinetic resolution strategies for the production of $\beta$-arylalanines employing enzymes with PAM activity.

Drawing on the established PAL catalyzed amination strategies that allow arylacrylates to be aminated directly with free ammonia at the $\alpha$-position, researchers envisaged the exploitation of the second half of the aminomutase reaction as a complementary strategy to access the $\beta$-amino acids. This was shown to be possible with the (R)-selective TwPAM from Taxus wallichiana var. chinensis using a 1200-fold excess of ammonia with trans-cinnamic acid, giving a $\beta: \alpha$ product distribution of around 50:50 (cis-cinnamic acid was not seen to be converted). ${ }^{263}$ Kinetic analyses of the reaction with cinnamate and various para-substituted analogues revealed that the enzyme gave higher $k_{\text {cat }}$ values and a lower $\beta: \alpha$ product ratio when EWGs (nitro and halogens) were present on the ring, as compared with EDGs (methyl and methoxy). This was reasoned to be due to the electronic effects of these substituents (Scheme 42), with the strongly activating $p$-nitro group priming the $\alpha$-position of the molecule for nucleophilic attack, an effect mitigated by a $p$ methoxy substituent, which would favor instead activation of the $\beta$-position by the more weakly 
withdrawing carboxyl moiety. ${ }^{263}$ Further analyses of $k_{\text {cat }}$ and $K_{\mathrm{M}}$ with an expanded substrate panel of 17 ring-substituted cinnamate derivatives revealed that the enzyme accepted a wider range of chemical groups, at the para-position, compared to the ortho- and meta-. This was reported to suggest a larger cavity around this portion of the substrate in the active site of the enzyme, allowing accommodation particularly of more bulky hydrophobic groups ${ }^{264}$ In the vast majority of cases, the enantiomeric excess values that could be determined at the end of the kinetic analyses were found to be $>99 \%$, with all $\beta$-products being $(R)$-configured and all $\alpha$-products $(S)$-, as expected. A comparison of Hammett constants for each substituent with the initial percentage of $\beta$-isomer seen with the corresponding arylacrylate substrate showed a strong linear correlation, ${ }^{264}$ reinforcing earlier hypotheses regarding substrate electronic effects and regioselectivity. ${ }^{263}$
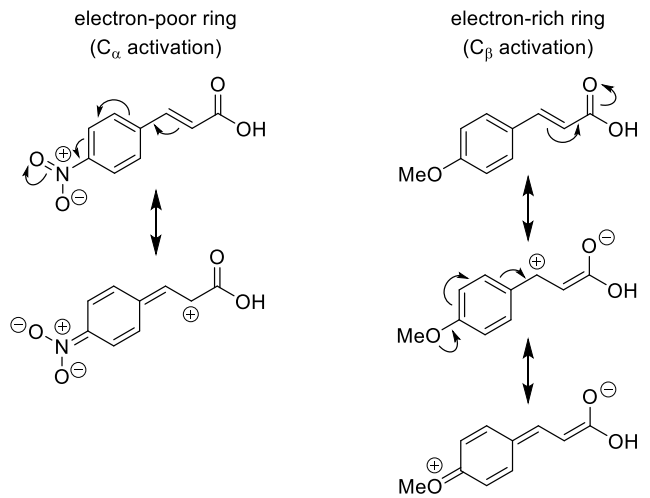

Scheme 42. Resonance forms of cinnamate derivatives with EWGs or EDGs on the aromatic ring in reference to activation of $\mathrm{C}_{\alpha}$ or $\mathrm{C}_{\beta}$.

Although the use of TwPAM as a hydroamination biocatalyst for the synthesis of $(R)-\beta$ phenylalanine derivatives was a promising asymmetric method, several challenges had to be overcome in order to make it a viable alternative to other routes. One of these was the presence of the unwanted $(S)$ - $\alpha$-amino acid in many cases, complicating any subsequent work-up procedures. 
A clever idea to circumvent this problem was proposed by Verkuijl et al., based on the addition of $\mathrm{PdCl}_{2}\left(\mathrm{PPh}_{3}\right)_{2}$ dissolved in dichloromethane for the selective extraction of the $\alpha$-amino acid in the organic layer, leaving the desired $\beta$-isomer in the aqueous phase. This isomer-selective liquidliquid extraction (ILLE) method was reported specifically for aminomutase reactions (in this case catalyzed by TwPAM) and has been shown to allow recovery of enantiopure $(R)$ - $\beta$-phenylalanine in $72 \%$ yield with up to $96 \%$ purity. ${ }^{265}$ Despite the utility of this regioisomeric separation, the underlying regioselectivity of the biocatalyst contributed to the low efficiency of the process, with a significant amount of the starting material being converted to side-product. To address this, efforts were made to design a regioselective enzyme to catalyze selective $\beta$-addition to the substrate and thus circumvent subsequent purification. As the electronic activation of the $\beta$ position for amination was thought to involve charge delocalization into the aromatic ring or the carboxylate group of each substrate, it was reasoned that targeted mutagenesis of the active site would affect the ability of the enzyme to stabilize these. As such, the aromatic ring and carboxylate binding portions of the TwPAM active site were subjected to pairwise site-saturation mutagenesis (CASTing). The resulting variants were screened and hits with enhanced $\beta$ regioselectivity identified in both the aryl and carboxylate binding libraries. The best of these, TwPAM-Gln319Met, was shown to give an initial $\beta: \alpha$ product ratio of $88: 12$, an increased $k_{\text {cat }}$ and an almost 2 -fold increase in endpoint production of $(R)-\beta$-phenylalanine when compared to the wild-type enzyme (Scheme 43a). Oddly, the superior variation at Gln319, which is predicted to interact with the carboxyl of the substrate, was not thought to have an electronic effect like some of the other variants, but rather prime the enzyme-substrate complex for addition on the re face of $\mathrm{C}_{\beta}$ by stabilizing a rotated substrate conformation. ${ }^{178}$ 


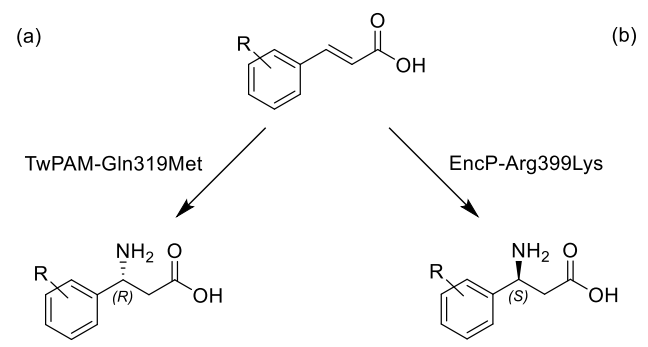

Scheme 43. Regioselective PAM variants allowing enantiocomplementary amination of arylacrylic acids to give ( $R$ )- $\beta$-phenylalanines (a) and ( $S)$ - $\beta$-phenylalanines (b).

Another issue with the TwPAM asymmetric synthetic method was the relatively low conversions achievable through use of this enzyme in industrially desirable time periods. Attempts with both the wild-type enzyme and the regioselective variant were found to give consistently incomplete conversion even when left for several days ${ }^{178,264,265}$ (around 60\% conversion at preparative scale after 4-5 days). As this problem was not evident among related PAL enzymes, ${ }^{139,151}$ studies of the molecular determinants of mutase-lyase activity within the protein family were drawn upon to address this. The two main differences thought to be characteristic of class I lyase-like ammonialyases and aminomutases are the substrate binding modes (allowing or disfavoring $\beta$-reamination as appropriate $)^{177,188}$ and the duration of ligand retention in the active site. Through temperature and viscosity studies in related enzymes with $(S)$-PAM activity, it has been reported that this second parameter is dictated, at least in part, by inner active site loop dynamics. ${ }^{193}$ With TwPAM acting physiologically to isomerize L-phenylalanine without releasing the cinnamate intermediate, it was seen as probable that the enzyme exhibited naturally slow opening and closing of the active site, a trait resulting in sluggish conversion in the synthetically useful amination reaction. To address this, molecular dynamics simulations of the lid opening were performed, revealing energetic barriers associated with breaking of two salt bridges involving adjacent arginine residues in the loop of TwPAM. Mutation of these to serine resulted in increased turnover for only one of the variants, with the other showing markedly decreased $k_{\text {cat }}$. 
The more successful TwPAM-Arg92Ser enzyme was found to allow PAL-like conversions of $87 \%$ and $96 \%$ after 1 and 3 days respectively, whilst retaining mutase-like regioselectivity. ${ }^{180}$ Further investigation of a known PAL sequence (PcPAL) demonstrated that this enzyme displays identical (Arg) or functionally equivalent (Glu/Asp) amino acids at positions homologous to the charge interaction residues in $(R)$-PAMs. These findings imply that such interactions are important for maintenance of the catalytic cycle (as demonstrated by the poorly active TwPAMArg93Ser variant $)^{180}$ and that nature has employed different strategies to alter loop dynamics. A comparison of the loop sequences from PcPAL and TwPAM showed several key differences, particularly in residue hydrophobicity and rigidity at certain positions, possibly contributing to the relative stability of the open (solvent-exposed) and closed (buried) conformations. Rational exchanges of many of these in TwPAM allowed enhancement of lyase activity with both regioisomers of phenylalanine, as well as 1.7-fold higher relative catalytic efficiency when starting with cinnamic acid. ${ }^{266}$ Interestingly, one of the variations inspired by the largest difference in hydrophobicity between the two enzymes Ile79Ser was reported to be detrimental to activity, supposedly due to unfavorable interactions with the catalytically essential Tyr80. ${ }^{266}$ However, the presence of serine does not seem to be detrimental in PcPAL and other characterized PAL enzymes are known to contain an isoleucine at the homologous position, ${ }^{112,135,191,267}$ which raises the possibility of this being a phylogenetic artefact, rather than a function discriminating difference.

Following on from the work with TwPAM, the possibility of using an enantiocomplementary enzyme in a similar manner was demonstrated with the discovery that the $(S)$-PAM AdmH can mediate production of both $\beta$ - and $\alpha$-phenylalanine, albeit with a conversion of about $1 \%{ }^{188}$ By changing the buffer to $4 \mathrm{M}$ ammonium sulfate with the related enzyme EncP, it was possible to achieve an identical $\beta: \alpha$ product ratio $(56: 44)^{188,268}$ but with $80 \%$ conversion and enantiomeric excess values determined to be $>95 \%(S)$ after $22 \mathrm{~h}$. As both $(S)$-selective enzymes were known to exhibit thermal mutase-lyase bifunctionality, ${ }^{193}$ the elevated incubation temperature of $55^{\circ} \mathrm{C}$ 
was found to give the optimal conversion for cinnamate as well as more variable conversion for 21 other ring-substituted analogues. ${ }^{268}$ Comparison of the percentage of the $\beta$-regioisomer product resulting from each biotransformation with the core electron binding energy shift ( $\triangle \mathrm{CEBE}$ ) values of the aromatic moiety of the substrate (previously calculated for each substituent and position $^{269}$ ) suggested a possible interplay between electronic effects and substrate positioning on regioselectivity. This prompted rational design of enzyme variants displaying enhanced amination preference, with one catalyst (EncP-Arg299Lys) giving complete $\beta$ selectivity with 9 of the 21 substrates and reduced amounts of the $\alpha$-regioisomer product in all but 5 cases (Scheme 43b). ${ }^{268}$ Interestingly, mutations at the position homologous to that changed in the $\beta$-selective TwPAM-Gln319Met resulted in the more $\alpha$-selective variants EncP-Gln293Met and EncP-Gln293Glu, which could be explained either by the alteration of substrate positioning, ${ }^{268}$ or possibly by an effect on charge stabilization as predicted previously. ${ }^{178}$

The various potential uses of class I lyase-like aminomutase enzymes as commercially-viable catalysts each have their own advantages and drawbacks. Arguably, the most value-added method is the isomerization of cheap, bio-derived aromatic amino acids to either of their enantiopure regioisomers. Although this has been demonstrated with the use of AdmH to manufacture tens of grams of $(S)-\beta$-phenylalanine, ${ }^{259,260}$ there are only reports of small scale syntheses of the $(R)-\beta$ forms of phenylalanine or tyrosine $e^{253,257}$ and, as of yet, no biocatalytic studies producing $(S)$ - $\beta$ tyrosine with any sort of process-relevant enantioselectivity. Expansion of the product scope via the introduction of non-natural phenylalanine analogues has also shown promise with the preparation of hundreds of milligrams at a time, when using an $(S)$-selective aminomutase in a whole cell formulation. ${ }^{249}$ However, this requires enantiopure starting materials which are less readily available than their natural counterparts. One way around this is the coupling of the reaction to a racemization step, allowing cheaper racemic substrates to be converted, although this has only been reported at a small scale with an $(R)$-selective aminomutase. ${ }^{255,256}$ Nondynamic kinetic resolutions have been reported with enantiocomplementary PAM-PAL cascades 
to allow preparative synthesis of both $(R)$ - $\beta$-phenylalanine and $(S)$-3-fluoro- $\beta$-phenylalanine from more easily accessible racemates. Although preparative in nature and easy to work up in an industrial setting, these methods do suffer from an upper yield limit of $50 \%,{ }^{262}$ which, depending on the value of the product, can be an undesirable feature. The direct amination of non-chiral arylacrylates represents an attractive asymmetric alternative that has been developed and demonstrated for a range of both $(R)$ - and $(S)$ - $\beta$-phenylalanine derivatives. However, the enantiocomplementary enzyme variants designed for these reactions are still not completely regioselective with some substrates owing to the inherent electronics of the reaction mechanism and have only been reported at small scale with $1-5 \mathrm{mM}$ concentration. ${ }^{178,268}$ In spite of the minority of industrial scale examples of aminomutase catalyzed processes, much of the ground work has been laid in enzymology and biocatalysis literature to allow future exploitation of this versatile group of catalysts through continued enzyme discovery, protein engineering, cascade design and process development.

\subsection{Enzymatic and chemo-enzymatic cascade applications}

The exponential rise of enzymatic reactions in industrial synthesis is set to rival traditional chemical multi-step syntheses in the near future. Nonetheless, while biocatalysis has gained enormous attention in the context of synthetic applications, it is unlikely to replace completely traditional chemical synthesis. By harnessing the robustness of chemical catalysis with the elegance of biocatalysis, new modular and efficient routes towards highly functionalized molecular scaffolds from simpler starting materials can be attained.

This has been demonstrated with PAL enzymes by developing telescopic routes to aromatic amino acids starting from the corresponding aromatic aldehydes. Successful synthesis of a range of halophenylalanines and arylalanines was carried out by Rétey and co-workers with a three-step chemo-enzymatic process (Scheme 44a). ${ }^{270}$ First, a Wittig-type reaction (Horner-WadsworthEmmons) in water using ethyl (triphenylphosphoranylidene)acetate converted the aldehyde to the 
corresponding ethyl cinnamate. Ester hydrolysis using pig liver esterase (PLE) followed, to afford the cinnamic acid. Lastly, after addition of ammonia and $\mathrm{pH}$ adjustment, purified PcPAL was added for the amination step. Full conversion to ethyl cinnamate was afforded from the Wittig reaction with a 95:5 mixture of $(E)$ and $(Z)$ diastereoisomers. While PLE successfully hydrolyzed both isomers, PAL, on the other hand, was specific for the $(E)$-isomer, leaving the $(Z)$-isomer untouched, thus leading to slightly decreased conversions. Furthermore, a major by-product of this process is triphenylphosphine oxide, notoriously difficult to remove from organic reactions. By employing an ion-exchange purification, halophenylalanines and heteroarylalanines were obtained with $72-91 \%$ isolated yields and $>98 \%$ ee over a period of $48-96 \mathrm{~h}$.

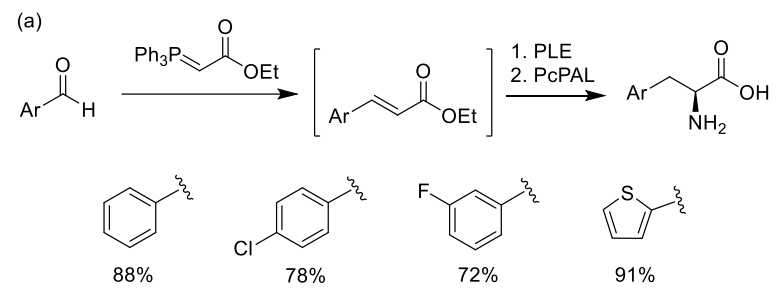

(b)

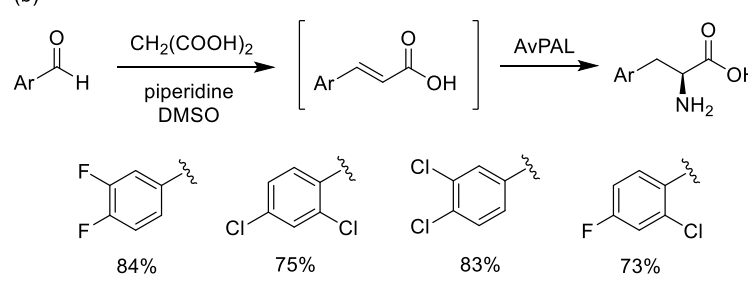

Scheme 44. One-pot chemo-enzymatic syntheses of arylalanines from the corresponding aldehydes via Wittig-type reaction, hydrolysis and amination (a) or Knoevenagel-Doebner condensation and amination (b).

We recently reported an alternative to this process, in this case using a Knoevenagel-Doebner condensation reaction for the synthesis of the cinnamic acid starting material (Scheme 44b), ${ }^{231}$ with improved atom economy and reduced waste generation (i.e., no formation of the $(Z)$-isomer or triphenylphosphine oxide). The reaction parameters for both the chemical and biocatalytic 
reactions were optimized for one-pot two-step operation; in particular switching the solvent from pyridine to DMSO proved crucial to avoid severe inhibition of the enzyme (AvPAL and RgPAL) in the biotransformation step. For the chemical step, microwave irradiation was employed to reduce reaction time and to afford greater control over the temperature. Experimental data revealed practically quantitative yields of the cinnamic acid only in an intermediate range of temperatures (with higher values consistently leading to lower conversions, likely due to DMSO decomposition in the presence of bases). The activity of RgPAL and AvPAL was tested towards halocinnamic acid analogs in the presence of varying DMSO concentrations, revealing a surprisingly high tolerance of PALs for this co-solvent (no significant inhibition was observed up to $10 \% \mathrm{v} / \mathrm{v}$ and reasonable activity was still present at $40 \% \mathrm{v} / \mathrm{v}$ ). This enabled substantially increased substrate loadings and productivities, leading to the one-pot telescopic synthesis of a number of halogenated amino acids in good to excellent conversions with high enantiopurity. RgPAL showed preference towards the mono-substituted substrates whilst AvPAL showed higher tolerance for bulkier dihalogenated substrates. Preparative scale synthesis of five amino acid precursors of different APIs afforded $71-84 \%$ isolated yields with $>98 \%$ ee. $^{231}$

By implementing the same telescopic process, novel ring-substituted heteroarylalanines were synthesized, including products containing EDGs. ${ }^{247}$ A panel of pyridylalanines was synthesized from the corresponding aldehydes with isolated yields of $32-65 \%$ with high enantiopurity. Several aldehydes from the picoline (ortho-), nicotine (meta-) and isonicotine (para-) series were incubated with AvPAL affording full conversion after 2-30 h (Scheme 45). While the conversion of each compound was high, the enantiopurity differed greatly between the three series. Low ee was obtained for the picoline and isonicotine isomers while the nicotine isomers generally gave high ee. The difference in optical purity was reported to be dependent on the electronic density of the aromatic system: electron-poor rings gave low ee compared to neutral or electron-rich ring systems, consistently with the alternative MIO-independent PAL mechanism postulated to afford the D-enantiomer ${ }^{154}$ (as discussed in Section 3.1). The addition of a strongly electron-donating 
methoxy substituent did not suppress the reactivity (EDG-substituted cinnamates are very poorly accepted by PALs), even though the observed ee profile suggests the electron-withdrawing power of the pyridine nitrogen is surpassed by the electron-donating ability of the methoxy group. This work sheds new light on the enantioselective addition of ammonia in the PAL catalyzed amination reaction, since electronic effects were shown to play an important role in determining the final enantiopurity of the product. In light of these results, we reported the optimum conditions to afford the highest enantiopurity by monitoring the biotransformation giving the best compromise between conversion, ee and purity, essential for the synthesis of chiral APIs. The demonstrated cost-saving measures of adopting chemo-enzymatic telescopic routes shows great potential for industrial application.

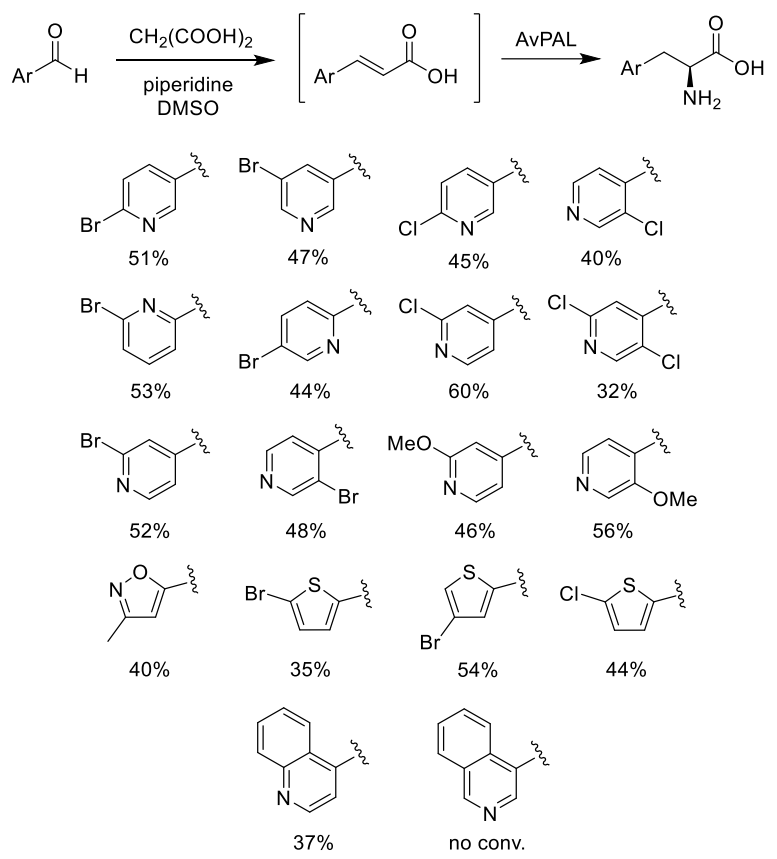

Scheme 45. Telescopic synthesis of a panel of heteroarylalanines (percentage values refer to isolated yields). 
Obviously, the benefits of chemo-enzymatic and multi-enzymatic catalysis for PAL reactions are not limited to the in situ synthesis of cinnamic acid precursor. Combining the PAL reaction with further downstream manipulations allows diversification of the range of non-natural amino acid derivatives, with the advantage of reducing the purification efforts and total process cost. Scheme 46 shows several examples of molecules of relevance for the pharmaceutical and agrochemical industry that have been synthesized with one-pot cascade/stepwise processes involving PALs. Except for the cascade synthesis of D-arylalanines (described in Section 4.3), the others will be described in the following.

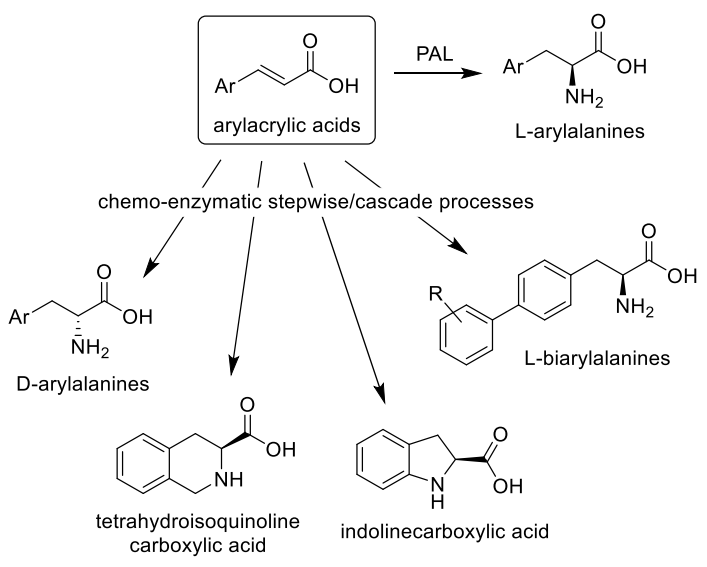

Scheme 46. Chemo-enzymatic one-pot stepwise/cascade processes involving PALs for the synthesis of industrially relevant phenylalanine derivatives.

Exploiting the reaction scope of metal catalysis in combination with stereospecific biocatalytic steps is a powerful strategy to access complex chiral scaffolds. However, the implementation of chemo-enzymatic processes usually requires a trade-off in the choice of solvent and conditions (chemical steps are normally run in organic solvents at high temperatures, while biocatalytic steps proceed in aqueous buffers close to room temperature), sometimes leading to one-pot two-step strategies where the conditions are modified in between, without purification of the intermediate. However, in the attempt to adhere even more to the principles of green chemistry, next-generation 
chemo-enzymatic processes for industrial production of fine chemicals will attempt to eliminate completely the use of organic solvents. This has been demonstrated by the chemical company DSM in one of the most frequently cited examples of industrial application of ammonia-lyases: the integration of the PAL reaction with a water-compatible metal catalyzed cyclization reaction in the conversion of 2-chlorocinnamic acid to $(S)$-2-indolinecarboxylic acid, ${ }^{271}$ a precursor of antihypertensives such as perindopril and indolapril (Scheme 47). The cinnamic acid was added to the biotransformation mixture in portions to prevent product inhibition, leading to $91 \%$ conversion and $>99 \%$ ee. To avoid possible racemization in the cyclization step and to reduce the overall cost of the process, copper was used instead of palladium as the metal catalyst. The best reaction parameters were found in neat water using $2 \mathrm{~mol} \% \mathrm{CuCl}$ giving full conversion to the cyclized product, affording (S)-2-indolinecarboxylic acid with 60\% isolated yield from 2chlorocinnamic acid with no loss of optical purity from the enzymatic step.

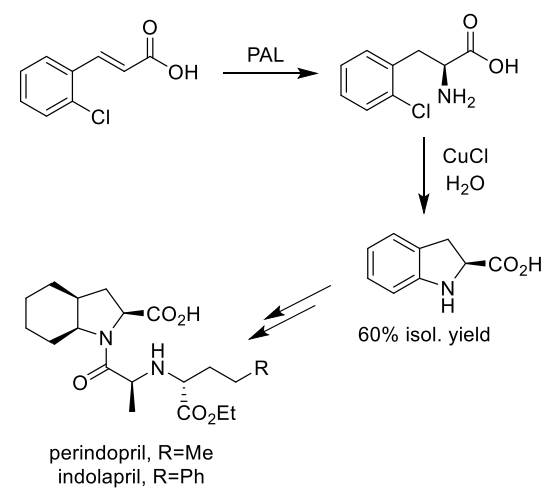

Scheme 47. PAL mediated chemo-enzymatic synthesis of (S)-2-indolinecarboxylic acid.

With a similar approach, we recently reported the chemo-enzymatic synthesis of $(S)-1,2,3,4$ tetrahydroisoquinoline-3-carboxylic acid via a PAL mediated biotransformation followed by a Pictet-Spengler reaction. ${ }^{272}$ The crude PAL mixture was lyophilized to remove water and ammonium carbonate, and the crude material used directly for the Pictet-Spengler reaction with 
concentrated aqueous formaldehyde in acidic conditions, to afford 53\% isolated yield of the enantiopure tetrahydroisoquinoline scaffold (Scheme 48).

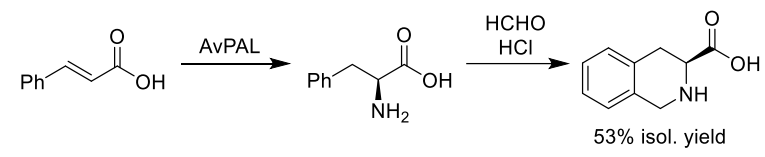

Scheme 48. Synthesis of (S)-1,2,3,4-tetrahydroisoquinoline-3-carboxylic acid by combining PAL biocatalytic step and a Pictet-Spengler reaction.

Another example from our group that highlights the power of chemo-enzymatic approaches, is the synthesis of biarylalanine API precursors by integrating the PAL mediated amination of $p$ bromocinnamic acid with the palladium catalyzed Suzuki-Miyaura coupling, a key chemical transformation widely used in medicinal chemistry (Scheme 49). ${ }^{273}$ To maximize the conversion of the biotransformation step the AvPAL-Phe107Ala variant was used, with a larger active site cavity to accommodate the bulky para-substituent. The palladium coupling reaction was optimized in neat water by screening different water-soluble palladium catalysts under microwave irradiation. However, a one-pot telescopic route was found not to be feasible, due to the high ammonia concentration inhibiting the palladium catalyzed step. Thus, the amino acid was isolated by adsorption on ion-exchange resin, before submitting it to a one-pot $N$-protection and Suzuki coupling. Employing a panel of arylboronic acids, a broad range of $N$-Boc-protected L-biarylalanines could be obtained with $>99 \%$ ee, in $33-65 \%$ isolated yield from the starting $p$ bromocinnamic acid (and with a different biocatalytic strategy the corresponding Dbiarylalanines were accessed as well). ${ }^{228}$ The synthetic value of this process was exemplified by synthesizing a DPP-IV inhibitor starting via the biarylalanine intermediate in 5 steps, with an overall isolated yield of $30 \%$ from $p$-bromocinnamic acid (Scheme 49). 


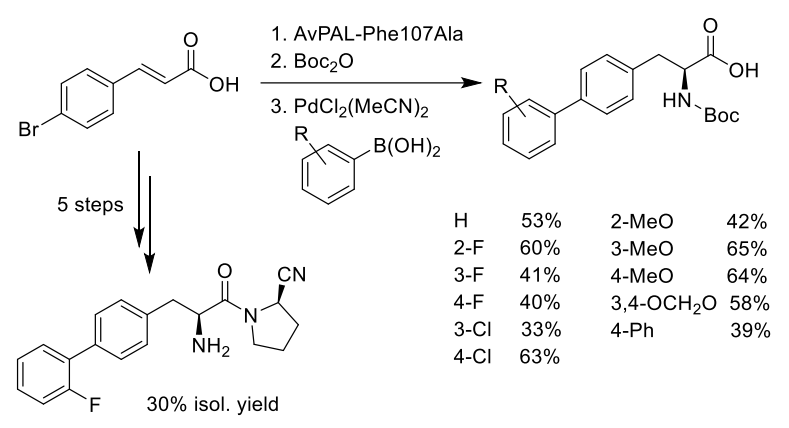

Scheme 49. Chemo-enzymatic synthesis of biarylalanines by coupling PAL biocatalytic synthesis with Suzuki-Miyaura coupling (percentage values refer to isolated yields).

While a huge amount of work on PALs was conducted in the reverse (non-natural) direction to synthesize high-value chiral amino acids, the natural reaction has not been overlooked for its synthetic utility, especially in the context of synthetic biology. The synthesis of cinnamic acid analogues from phenylalanine can provide a range of commercially relevant compounds by incorporating additional chemical and/or enzymatic steps. For example, $(S)$-styrene oxide, a key fine chemical towards functionalized chiral diols, was produced in vivo from glucose. PAL, transcinnamic acid decarboxylase (CADC) and styrene monooxygenase (SMO) were coexpressed in E. coli to afford the product epoxide from glucose via the primary metabolite phenylalanine (Scheme 50). ${ }^{274}$

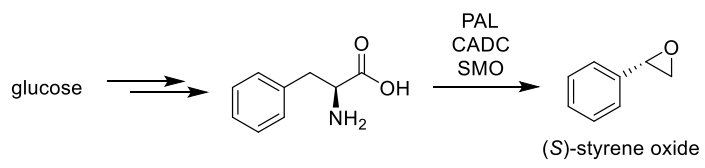

Scheme 50. In vivo production of $(S)$-styrene oxide from glucose via co-expression of PAL, CADC and SMO.

Using a similar strategy Kroutil and co-workers demonstrated the in vivo synthesis of a panel of vinylated phenols starting from simple substituted phenols. ${ }^{275}$ This multi-enzymatic cascade 
involved co-expression of tyrosine phenol-lyase from Citrobacter freundii (CfTPL), tyrosine ammonia-lyase from Rhodobacter sphaeroides (RsTAL) and ferulic acid decarboxylase from Enterobacter sp. (ExFAD). The first step of the cascade involves a C-C bond formation reaction between phenol and pyruvate catalyzed by a CfTPL variant with a relaxed substrate specificity to obtain L-tyrosine derivatives. RsTAL catalyzes the deamination of L-tyrosine to coumaric acid followed by decarboxylation by EsFAD to produce several different substituted vinylated phenols with $>99 \%$ conversion and isolated yields varying from $65-83 \%$ (Scheme 51).

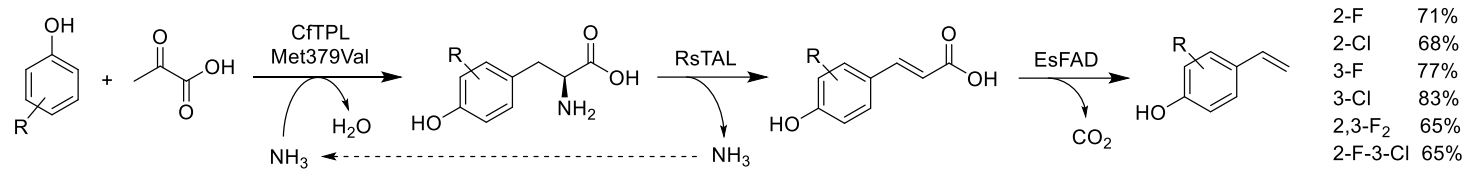

Scheme 51. Multi-enzymatic synthesis of vinylated phenols (percentage values refer to isolated yields).

A remarkable eight-step ten-enzyme cascade was reported by Li and co-workers, ${ }^{276}$ with PAL as the gateway to a variety of valuable chemical intermediates including styrene oxide, diols, $\alpha$ hydroxyacids and phenylglycine derivatives (Scheme 52). The enzymes involved are PAL, phenylacrylic acid decarboxylase (PAD), styrene monooxygenase (SMO), epoxide hydrolase (EH), alcohol dehydrogenase (ADH), aldehyde dehydrogenase (ALDH), hydroxymandelate oxidase (HMO), transaminase (TA), glutamate dehydrogenase (GluDH) and catalase (CAT). The enzymatic synthesis of enantiopure (S)-phenylglycine (eight enzymes for functional group manipulations, GluDH for cofactor regeneration and CAT for $\mathrm{H}_{2} \mathrm{O}_{2}$ removal) was achieved with $85 \%$ conversion and $>99 \%$ ee. The products were obtained from biomass feedstock, since bioderived L-phenylalanine was used as the starting material and the reactions were run in a biphasic mixture using bio-derived ethyl oleate from renewable resources as the organic solvent. 


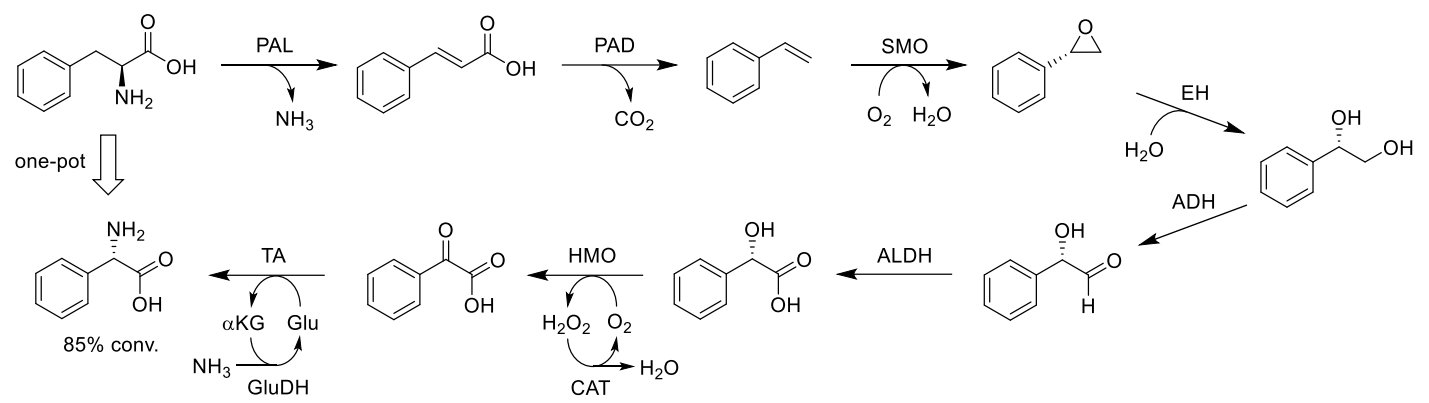

Scheme 52. Eight-step cascade for the synthesis of $(S)$-phenylglycine starting from the primary metabolite L-phenylalanine.

The advent of synthetic biology has revolutionized the in vivo production of fine chemicals, particularly in the areas of metabolic engineering and synthetic biology, and PALs/TALs show great potential towards the industrial scale production of secondary metabolites in microbial cell factories. The PAL reaction can be seen as the gatekeeping step to the phenylpropanoid biosynthetic pathway, thus a substantial amount of work has been carried out on the integration of these enzymes into different pathways and hosts to produce bio-derived fine chemicals from phenylalanine and tyrosine. A few representative examples are shown in Scheme 53. Katsuyama et al. co-produced PAL from Rhodotorula rubra, 4-coumaryl ligase from Lithospermum erythrorhizon and stilbene synthase from Arachis hypogaea in E. coli, affording $20 \mathrm{mg} \mathrm{L}^{-1}$ of pinosylvin and $37 \mathrm{mg} \mathrm{L}^{-1}$ resveratrol from $490 \mathrm{mg} \mathrm{L}^{-1}$ phenylalanine and $540 \mathrm{mg} \mathrm{L}^{-1}$ tyrosine respectively. ${ }^{277}$ A similar approach was employed by Nielsen and co-workers by introducing the phenylpropanoid biosynthetic pathway into Saccharomyces cerevisiae. By controlling the metabolic fluxes through overexpression of the genes for the enzymes involved in malonyl-CoA synthesis and the feedback-inhibition resistant versions of 3-deoxy-D-arabinoheptulosonate-7phosphate (DAHP) synthase and chrorismate mutase, a 20,000-fold improvement in resveratrol yield from glucose was obtained. ${ }^{278}$ To improve the conversion further, Li et al. incorporated the resveratrol biosynthetic pathway from Arabidopsis thaliana and resveratrol synthase from Vitis vinifera in S. cerevisiae, producing $800 \mathrm{mg} \mathrm{L}^{-1}$ of resveratrol. ${ }^{279}$ Styrene was also produced by 
employing a similar method, using hybrid PAL isoforms from Populus kitakamiensis. ${ }^{280}$ Moriguchi and co-workers reported on the production of $9.4 \mathrm{mg} \mathrm{L}^{-1}$ chrysin from tyrosine in $E$. coli. ${ }^{281}$ Ververidis and co-workers highlighted the production of quercetin by coexpressing eight genes in yeast. The strain was tested for its ability to produce quercetin by supplementing the growth media with intermediate substrates in the phenylpropanoid pathway; naringenin, $p$ coumaric acid and phenylalanine. Naringenin and $p$-coumaric afforded $0.38 \mathrm{mg} \mathrm{L}^{-1}$ and $0.26 \mathrm{mg}$ $\mathrm{L}^{-1}$ of quercetin respectively while phenylalanine gave no traceable amount of product. The authors postulated that a suboptimal spatial arrangement of metabolic enzymes (referred to as a metabolon) may have been present in this system, hence affording poor yield of the final product. ${ }^{282}$ Furthermore, to counteract the lack of highly active and specific TALs for metabolic engineering applications, a recent enzyme discovery and comparative in vivo study was carried out, leading to a panel of new TALs that show potential for production of $p$-coumarate and related phenylpropanoids in different strains of bacteria and yeasts. ${ }^{283}$ Indeed, by harnessing the power of metabolic engineering and synthetic biology, these and a large number of other challenging target molecules could be obtained with PALs/TALs in vivo, as extensively covered in a very recent review. ${ }^{20}$ 


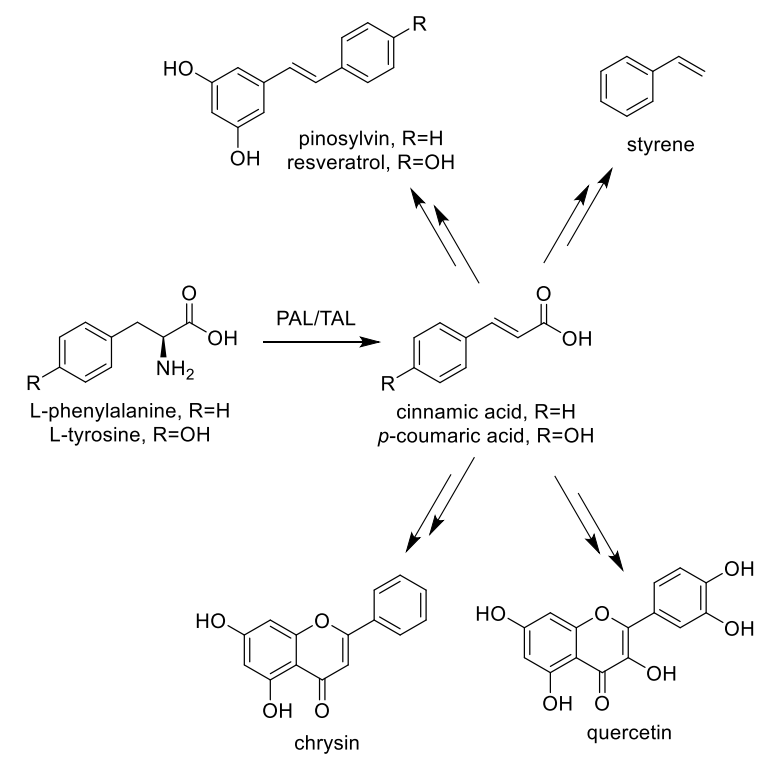

Scheme 53. PAL/TAL mediated production of secondary metabolites through metabolic engineering and synthetic biology.

\section{Therapeutic applications of ammonia-lyases}

The use of enzymes as biotherapeutics is a broad area of study that takes advantage of the physiologically compatible conditions and biomedically relevant substrate specificity of various biological catalysts. Ammonia-lyases are particularly suited for medical use, due to their activity on primary metabolites, often strict substrate preference and lack of requirement for cofactor supplementation. They are also known generally to have higher specific activities than the related (but equally selective) aminomutase enzymes ${ }^{192,193,266}$ and produce benign products such as cinnamate, $p$-coumarate or urocanate and trace amounts of ammonia. One area that has been researched for a long time is the use of ammonia-lyase enzymes in potential cancer treatments. This use stems from the knowledge that tumor cell proliferation is fuelled largely by the bloodborn substrate pool of primary metabolites, which includes all proteinogenic amino acids. Whilst some of these protein building blocks can be obtained through cellular metabolism from other 
starting materials, many cannot be produced by human metabolic processes and are therefore taken up by proliferating cells. The suite of amino acids required for growth varies between cell types and is therefore not uniform across different forms of cancer. However the majority of human cells require nine 'essential' amino acids that are sourced from dietary protein and thus depletion of these in the blood plasma is a promising strategy to restrict uncontrolled cell division. ${ }^{284}$ Of the natural ammonia-lyase substrates, both histidine and phenylalanine cannot be produced by human metabolism, whereas tyrosine can be synthesized, but only via selective hydroxylation of phenylalanine. However there are also specific cancer types, such as melanoma, which have been shown to be sensitive to restriction of tyrosine. ${ }^{285,286}$ As such, the use of these enzymes for the selective removal of an aromatic amino acid provides a means by which tumor progression can be slowed down or halted with minimal effect to non-cancerous resting cells. ${ }^{13,287}$

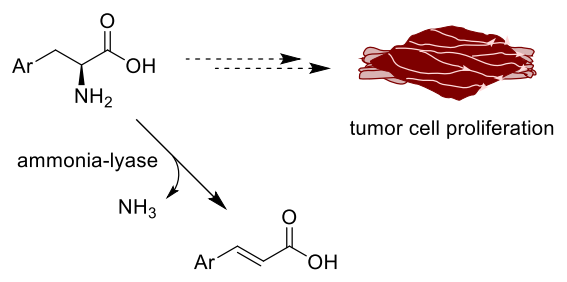

Scheme 54. The use of ammonia-lyases to remove aromatic amino acid nutrients in vivo and halt abnormal cell growth.

The first examples of such research were published several decades ago and detailed the use of a bifunctional PAL/TAL to halt protein production, subsequently inhibiting RNA/DNA synthesis and thus cell division and viability in mouse leukemic lymphocytes and lymphoblasts. ${ }^{28-290}$ Similarly the use of a bacterial HAL was shown to give antineoplastic effects ${ }^{291}$ and additionally allow the selective repression of murine sarcoma cell proliferation, ${ }^{292}$ possibly due to a similar mechanism. However, the transfer of the technology from cell culture to animal studies highlighted immunogenic issues with the introduction of the foreign proteins along with their 
rapid clearance, even after repeated injections. ${ }^{292,293}$ It is possible that these undesirable properties hindered the further development of ammonia-lyases as anticancer agents, as there seem to be no further reports of their use until relatively recently. One such example discloses the use of a more recently discovered HAL to control of cell growth in prostate and ovarian cancer cell lines by removing histidine as a precursor for protein synthesis and histamine production. ${ }^{294,295}$ This work also details the potential of HAL mediated metabolite depletion as a means of inducing reversible arrest of the cell cycle in normal tissues, thus allowing unaffected tumor cells to be targeted more selectively by anticancer drugs. ${ }^{294,295}$ There has also been commercial interest in the use of prokaryotic PALs (rather than fungal enzymes with both PAL and TAL activity), as demonstrated by a patent from the company BioMarin Pharmaceutical Inc. ${ }^{296}$ which specializes in enzyme replacement therapies. The patent describes extensive study of a previously described soluble variant of the PAL from Anabaena variabilis ${ }^{130}$ and various chemical conjugate formulations showing stability at physiological temperature, slow clearance in vivo (in rat and macaque) and favorable $\mathrm{IC}_{50}$ values for liver, skin, prostate, lung, colon, brain and blood cancer cell lines. ${ }^{296}$ The most recent example from 2013 makes use of a fungal PAL/TAL from Rhodosporidium toruloides, a close relative of the enzyme used 40 years earlier. This homologue was found to have good catalytic properties (turnover, $K_{\mathrm{M}}$, thermostability, $\mathrm{pH}$ tolerance) as well as significant cytotoxicity against breast and prostate cancer tissues. ${ }^{297}$ Although in depth toxicological studies have not been undertaken, this finding highlights the importance of assaying various protein candidates from different organisms to exploit the natural diversity in enzyme traits desirable for medical intervention.

Another area where class I lyase-like enzymes could be of commercial interest are pathological imbalances in primary metabolites. These can include inborn errors of metabolism where genetic defects result in abnormal enzyme activities, causing flux through biosynthetic and catabolic pathways to be obstructed or altered detrimentally. A notable example of this is phenylketonuria (PKU), an irregularity in an aromatic amino acid hydroxylase (AAAH) or its cofactor recycling 
apparatus, which results in accumulation of phenylalanine and low levels of tyrosine in the body (Scheme 55). The high concentration of L-Phe in the blood, known as hyperphenylalaninemia, has been proposed to be the cause of several PKU symptoms, including intellectual impairment, microcephaly, seizures and congenital heart defects. ${ }^{298-302}$ There are also several diseases associated with faulty tyrosine metabolism, resulting in increased concentrations of this amino acid and various other associated compounds. The most prominent of these is fumaryl acetoacetase deficiency, which affects multiple organ systems often resulting in neurological disorders, respiratory, kidney and liver failure as well as defective blood clotting and gastrointestinal problems (diarrhoea, vomiting, excretion of blood). Other examples include type III tyrosinemia, a rare disease severely affecting the nervous system, the Richner-Hanhart syndrome, characterized by eye sensitivity, painful skin lesions and intellectual disability, and alkaptonuria, which results in skin or cartilage damage and sometimes arthritis. There are also non-hereditary instances, such as vitamin $\mathrm{C}$ deficiency and premature birth, that can result in heightened blood tyrosine and various associated symptoms. ${ }^{303}$

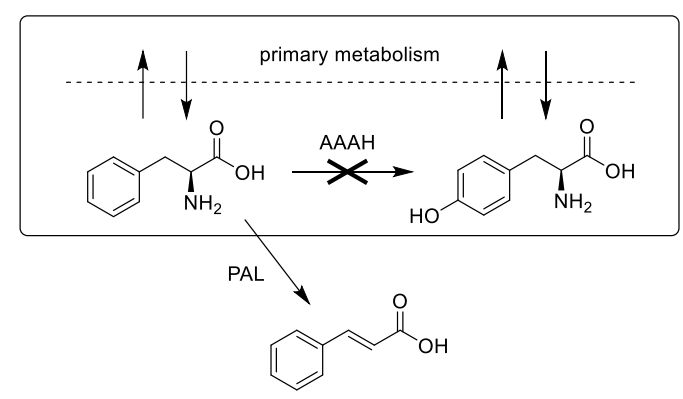

Scheme 55. The use of PAL to treat abnormalities in aromatic amino acid hydroxylase (AAAH) by removing accumulating phenylalanine.

Enzymes with PAL activity have been widely studied for the treatment of PKU, with various methods employed to improve the biotherapeutic properties of a handful of proteins. Although some work exists on the treatment of foodstuffs by PAL to reduce dietary phenylalanine ${ }^{304}$ and 
thus hyperphenylalaninemia, most research aims to use the enzymes within the body. This is because current treatments for PKU already encompass strict dietary restrictions which are difficult to follow and/or implement in patient groups. ${ }^{299,301,302}$ There exist two key methods for administration of PAL as a treatment: subcutaneous injection, which aims to reduce phenylalanine levels directly in blood plasma, and oral administration, a method which would remove dietary phenylalanine in the gastrointestinal tract after proteolytic release but before absorption. The dissimilar environments encountered by a therapeutic biomolecule in these two scenarios warrant optimization of different characteristics, often using the same starting enzyme. For example, a PAL formulation intended for injection requires careful consideration of immunogenic properties as well as high stability and activity at blood $\mathrm{pH}$. Tailoring a biocatalyst for use as a dietary supplement, however, would need to focus on high stability and sustained activity at the various $\mathrm{pH}$ values encountered in the digestive system as well as resistance to human proteolytic enzymes. The two main methods evident in the literature for achieving such alterations to wild-type enzymes are via formulation development (including chemical conjugation, immobilisation or encapsulation of the enzyme for general stability) or through traditional enzyme engineering techniques, which focus on specific desirable features. A summary of some of the more notable examples of this is presented in Table 4. One of the most advanced studies of utilizing PAL enzymes, spanning almost a decade, is the development of polyethylene glycol-enzyme conjugates with vastly improved traits for use as an injectable PKU treatment. ${ }^{296,298,299,301,302,305,306}$ A preparation of PEGylated AvPAL from BioMarin Pharmaceutical (with the trade name pegvaliase) has even been taken through to human clinical trials with results from single-dose injections being well tolerated by adult patients with PKU, although all participants were observed to produce an immune response to polyethylene glycol. ${ }^{307}$ Pegvaliase recently entered phase 3 clinical trials. 
Table 4. Methods employed to improve the therapeutic properties of PAL enzymes from different organisms.

\begin{tabular}{lll}
\hline Enzyme & Method & Ref. \\
\hline AvPAL & Loaded into non-immunogenic red blood cells (erythrocytes) from human & 300 \\
AvPAL & Cys503Ser-Cys565Ser variant with reduced aggregation & 130 \\
AvPAL & Phe18Ala variant altering human chymotrypsin cleavage site & 306 \\
AvPAL & Phe18Ala-Cys503Ser-Cys565Ser variant conjugated to 5-kDa linear polyethylene glycol & 299 \\
AvPAL & Silica gel immobilization and conjugation to 5-kDa polyethylene glycol & 306 \\
AvPAL & Gln292Cys variant reducing chemical unfolding & 308 \\
PcPAL1 & Lactobacillus lactis whole cells within calcium alginate mircroparticles & 309,310 \\
PcPAL1 & Conjugated to 2,4-bis $(O$-methoxypolyethyleneglycol)-6-chloro-s-triazine & 302 \\
RgPAL & 10\% haemoglobin water-saturated ether encapsulated in nitrocellulose & $311,312,313$ \\
RtPAL & Conjugated to linear 20-kDa polyethylene glycol & 298,314 \\
RtPAL & Entrapped in ultra-large-pore mesoporous silica & 315 \\
AvPAL & Phe18Ala-Cys503Ser-Cys565Ser in multi-compartment liposomal microreactors & 316 \\
RgPAL & Cross-linked enzyme aggregates with glutaraldehyde & 317 \\
RgPAL & Cross-linked enzyme aggregates with crude-pored microspherical silica core & 318 \\
RgPAL & Gln137Glu variant with greater stability at acid pH & 319 \\
RtPAL & Cross-linking and variation at 8 positions to reduce proteolytic cleavage & 320 \\
\hline
\end{tabular}

In terms of mutagenesis for the optimisation of ammonia-lyases, one of the most in depth studies is reported as evidence for two patents held by Codexis, Inc., an enzyme technology company. ${ }^{303,321}$ Although several sequences of prokaryotic PAL and TAL enzymes are covered by these, most of the work pertains to large scale mutagenesis of AvPAL for PKU treatment ${ }^{321}$ and the same enzyme incorporating the Phe107His substrate-switching variation ${ }^{107}$ for pathologies associated with abnormal tyrosine metabolism. ${ }^{303}$ Both patents detail the screening of an extensive library of AvPAL variants with respect to substrate specificity for phenylalanine vs. tyrosine, resistance to animal and human proteases, as well as tolerance to pancreatic extract ${ }^{303}$ and/or intestinal detergents. ${ }^{321}$ These studies represent one of the most promising strategies to obtain ammonia-lyases suitable for oral administration to treat metabolic abnormalities. Additionally the TAL-specific patent includes investigation of applicability for lowering blood tyrosine within a tyrsoinemia mouse model and in silico identification for additional mutagenesis of T-cell specific epitopes to create variants with reduced immunogenicity, ${ }^{303}$ possibly to find candidates for subcutaneous administration. Interestingly, this work constitutes a rare example of application of an enzyme with TAL activity to treat an error in tyrosine metabolism, an application that is not widely researched despite the many examples of associated diseases. 
Aside from alleviation of unwanted proteinogenic amino acid accumulation, there are also reports of more wide-ranging therapeutic uses of ammonia-lyase enzymes. The discovery the TAL from Rhodobacter sphaeroides is able to deaminate the neurotransmitter precursor Ldihydroxyphenylalanine (L-DOPA) ${ }^{132}$ led to speculations of its potential use to treat imbalances in neurochemicals, such as Tourette's syndrome and certain forms of schizophrenia. ${ }^{322}$ L-DOPA is also used as a pharmaceutical to treat Parkinson's disease by increasing the amount of dopamine that can be produced by brain localized enzymes. ${ }^{322}$ Therefore the use of RsTAL to remove the drug from other parts of the body in a site-specific manner could provide better control during therapy. Interestingly, the deamination product of L-DOPA (caffeic acid) is known to have antioxidant and tumor-suppressing qualities, which could allow TALs to be used as biotherapeutics in related pathologies. ${ }^{322}$ Another example, similar to the production of caffeic acid, is the in situ generation of trans-urocanate from readily available L-histidine, followed by conversion of this to the cis-diastereoisomer using ultraviolet light, ${ }^{294,295}$ presumably in peripheral tissues (Scheme 56). It is reported that this can allow the immunosuppressant properties of the isomerized product to be exploited where required, combatting rapid clearance usually seen when cis-urocanate is introduced alone. ${ }^{294,295}$ Another use of HAL, listed in the same study, is as an enhancer of the pharmaceutical properties of the compound L-histidinol. It is reported that, although L-histidinol could be an effective antiviral agent in the context of various highly infectious human pathogens, its effect is reduced in the presence of the primary metabolite Lhistidine, minimizing its use in vivo. However, removal of the competing amino acid using a HAL enzyme has not been possible until recently, due to the apparent inhibition of this class of enzyme by L-histidinol (Scheme 56). ${ }^{294}$ Characterization of a novel HAL from Kurthia sp. (KxHAL) revealed it to retain activity in the presence of the inhibitor. Indeed initial application of KxHAL in vitro has shown reductions in plaque growth and number for both herpes simplex virus (HSV) and respiratory syncytial viruses (RSV) in standard virology experiments. ${ }^{294,295}$ This enzyme-drug combination was also linked to inhibition of reverse transcriptase in Rauscher 
murine leukemia virus (RMuLV), an enzyme known to be required for infection in this family of viruses, which includes human immunodeficiency virus (HIV). ${ }^{295}$

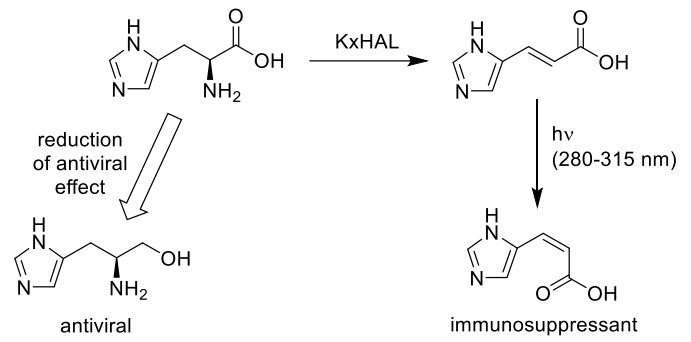

Scheme 56. Potential medical uses of the HAL from Kurthia sp. in conjunction with either Lhistidinol or ultraviolet irradiation.

\section{Conclusions and perspectives}

In view of the importance of amino acids as pharmaceutical and agrochemical intermediates, methods for their synthesis, particularly on scale, are likely to increase in demand in the future. In many cases the production of these building blocks will need to be carried out in an economic and sustainable manner, delivering products of high stereochemical purity at low cost. This challenge provides significant opportunities for the development of engineered biocatalysts, and in particular ammonia-lyases and aminomutases which, as demonstrated above, possess the potential to catalyze production of this class of molecules with high atom efficiency and enantioselectivity starting from inexpensive raw materials. Although some industrial processes based upon ammonia-lyases and aminomutases are starting to emerge, more research is needed to expand the substrate scope of these enzymes as well increases in stability and activity, so that they are able to tolerate the high substrate concentrations that are required on scale.

Ammonia-lyases and aminomutases have been shown to be suitable for cascade reactions, enabling sequential transformations to be carried out in a one-pot manner. The rapid 
developments in synthetic biology and metabolic pathway engineering are likely to witness greater application of these enzymes as key components of multi-step biocatalytic processes, in order to either create non-natural amino acids in vivo, or alternatively use amino acids as renewable feedstocks for conversion to other high value products.

Furthermore, several promising biotherapeutic applications have been demonstrated for specific ammonia-lyase classes, leading to a whole range of new treatment strategies for different diseases and conditions (most prominently phenylketonuria and several forms of cancer). Some of these have reached advanced stages in clinical trials and have been proven superior to established methods. Due to improved efficiency, in conjunction with their favorable combination of properties, engineered ammonia-lyases are expected to become considerably more relevant in the development of next-generation therapeutic strategies in the near future.

\section{Biographies}

Fabio Parmeggiani graduated with honors in Chemical Engineering in 2009 at Politecnico di Milano (Italy), under the supervision of Professor Claudio Fuganti. He obtained his PhD in Industrial Chemistry and Chemical Engineering in 2013, working in the group of Professor Elisabetta Brenna and Professor Francesco G. Gatti, focusing on the development of synthetic applications of biocatalytic reductions mediated by yeasts and isolated ene-reductases. He was also a visiting student at the Department of Biosystems Science and Engineering of the ETH-Z (Basel, Switzerland) in the group of Professor Sven Panke, working on the production and purification of ene-reductases from yeasts. After his $\mathrm{PhD}$, he moved to the Manchester Institute of Biotechnology (MIB) of the University of Manchester, to work as a research associate with

Professor Nicholas J. Turner on the development and evolution of ammonia-lyases for the synthesis of amino acid derivatives. He is author of more than 50 publications and his current research interests include the design and exploitation of chemo-enzymatic cascades for synthetic 
organic chemistry, the directed evolution of biocatalysts for industrial applications and the development of novel high-throughput assay systems.

Nicholas J. Weise joined the Faculty of Life Sciences at the University of Manchester to read molecular biology in 2009. During his studies he was awarded summer research studentships at the MRC Laboratory of Molecular Biology (Cambridge), the Institute of Cancer Research (London) and the Wellcome Trust Sanger Institute (Hinxton). In 2012 he graduated with a first class BSc (Hons) and University Award for Outstanding Academic Achievement before beginning a doctoral program at the Centre of Excellence in Biocatalysis, Biotransformation and Biocatalytic Manufacture (CoEBio3), part of the University's School of Chemistry. He finished his $\mathrm{PhD}$ in 2015, submitting a thesis on the discovery and engineering of class I lyase-like enzymes for the synthesis of unnatural amino acids, under the supervision of Professor Nicholas J. Turner. Since then, he has continued in the department as a postdoctoral teaching and research associate, whilst also managing the public engagement program at the Manchester Institute of Biotechnology (MIB). His interests include identification and demonstration of novel biocatalysts for sustainable chemical manufacture and promoting public understanding of and involvement with science.

Syed T. Ahmed joined the School of Chemistry at the University of Manchester in 2009 reading Medicinal Chemistry. During this time he was awarded the Nuffield scholarship to work at the Manchester Institute of Biotechnology (MIB) on carbohydrate synthesis. He completed his final project in 2013 working on supramolecular chemistry in Professor David Leigh's group, obtaining his MChem in the same year. This was followed by starting his $\mathrm{PhD}$ in the group of Professor Nicholas J. Turner and Professor Sabine L. Flitsch, working in the field of industrial biocatalysis. His $\mathrm{PhD}$ thesis focuses on the discovery, evolution and application of phenylalanine ammonia-lyases and is due to be submitted in 2017. He is dedicated to the development and 
application of sustainable technologies towards a green bio-economy and is the managing director of Stratazyme, a market research company dedicated to developing sustainable solutions.

Nicholas J. Turner obtained his DPhil in 1985 with Professor Sir Jack Baldwin and from 19851987 was a Royal Society Junior Research Fellow, spending time at Harvard University with Professor George Whitesides. He was appointed lecturer in 1987 at Exeter University and moved to Edinburgh in 1995, initially as a Reader and subsequently Professor in 1998. In October 2004 he joined Manchester University as Professor of Chemical Biology where his research group is located in the Manchester Institute of Biotechnology Biocentre (MIB). He is Director of the Centre of Excellence in Biocatalysis (CoEBio3) and a Co-Director of SYNBIOCHEM, the BBSRC Synthetic Biology Research Centre. He is also a co-founder of Ingenza. His research interests are in the area of biocatalysis with particular emphasis on the discovery and development of novel enzyme catalysed reactions for applications in organic synthesis. His group are also interested in the application of directed evolution technologies for the development of biocatalysts with tailored functions.

\section{$\underline{\text { ORCIDs }}$}

Fabio Parmeggiani: 0000-0001-5861-9269

Nicholas J. Weise: 0000-0003-3363-9102

Syed T. Ahmed: 0000-0001-8845-7440

Nicholas J. Turner: 0000-0002-8708-0781 


\section{$\underline{\text { References }}$}

(1) Viola, R. E. L-Aspartase: New Tricks from an Old Enzyme. In Advances in Enzymology and Related Areas of Molecular Biology, Vol. 74; 2000; pp 295-341.

(2) De Villiers, M.; Veetil, V. P.; Raj, H.; De Villiers, J.; Poelarends, G. J. Catalytic Mechanisms and Biocatalytic Applications of Aspartate and Methylaspartate Ammonia Lyases. ACS Chem. Biol. 2012, 7, 1618-1628.

(3) Mizobata, T.; Kawata, Y. Aspartases: Molecular Structure, Biochemical Function and Biotechnological Applications. In Industrial Enzymes: Structure, Function and Applications; 2007; pp 549-565.

(4) Veetil, V. P.; Fibriansah, G.; Raj, H.; Thunnissen, A.-M. W. H.; Poelarends, G. J. Aspartase/Fumarase Superfamily: A Common Catalytic Strategy Involving General BaseCatalyzed Formation of a Highly Stabilized Aci-Carboxylate Intermediate. Biochemistry 2012, 51, 4237-4243.

(5) Levy, C. W.; Buckley, P. A.; Sedelnikova, S.; Kato, Y.; Asano, Y.; Rice, D. W.; Baker, P. J. Insights into Enzyme Evolution Revealed by the Structure of Methylaspartate Ammonia Lyase. Structure 2002, 10, 105-113.

(6) Asuncion, M.; Blankenfeldt, W.; Barlow, J. N.; Gani, D.; Naismith, J. H. The Structure of 3-Methylaspartase from Clostridium tetanomorphum Functions via the Common Enolase Chemical Step. J. Biol. Chem. 2002, 277, 8306-8311.

(7) Babbitt, P. C.; Hasson, M. S.; Wedekind, J. E.; Palmer, D. R. J.; Barrett, W. C.; Reed, G. H.; Rayment, I.; Ringe, D.; Kenyon, G. L.; Gerlt, J. A. The Enolase Superfamily: A General Strategy for Enzyme-Catalyzed Abstraction of the $\alpha$-Protons of Carboxylic Acids. Biochemistry 1996, 35, 16489-16501.

(8) Herrmann, G.; Selmer, T.; Jessen, H. J.; Gokarn, R. R.; Selifonova, O.; Gort, S. J.; Buckel, W. Two $\beta$-Alanyl-CoA:Ammonia Lyases in Clostridium propionicum. FEBS J. 2005, 272, 813-821.

(9) Heine, A.; Herrmann, G.; Selmer, T.; Terwesten, F.; Buckel, W.; Reuter, K. High Resolution Crystal Structure of Clostridium propionicum $\beta$-Alanyl-CoA:ammonia Lyase, a New Member of the "Hot Dog Fold" Protein Superfamily. Proteins: Struct. Funct. Bioinf. 2014, 82, 2041-2053.

(10) Jeng, I.; Barker, H. A. Purification and Properties of L-3-Aminobutyryl Coenzyme A Deaminase from a Lysine-Fermenting Clostridium. J. Biol. Chem. 1974, 249, 6578-6584.

(11) Barker, H. A.; Kahn, J. M.; Chew, S. Enzymes Involved in 3,5-Diaminohexanoate Degradation by Brevibacterium sp. J. Bacteriol. 1980, 143, 1165-1170.

(12) Turner, N. J. Ammonia Lyases and Aminomutases as Biocatalysts for the Synthesis of $\alpha-$ Amino and $\beta$-Amino Acids. Curr. Opin. Chem. Biol. 2011, 15, 234-240.

(13) Heberling, M. M.; Wu, B.; Bartsch, S.; Janssen, D. B. Priming Ammonia Lyases and Aminomutases for Industrial and Therapeutic Applications. Curr. Opin. Chem. Biol. 2013, 17, 250-260.

(14) Cooke, H. A.; Christianson, C. V; Bruner, S. D. Structure and Chemistry of 4Methylideneimidazole-5-One Containing Enzymes. Curr. Opin. Chem. Biol. 2009, 13, 460-468.

(15) MacDonald, M. J.; D’Cunha, G. B. A Modern View of Phenylalanine Ammonia Lyase. Biochem. Cell Biol. 2007, 85, 273-282. 
(16) Kumavath, R. N.; Ramana, C. V.; Sasikala, C.; Barh, D.; Kumar, A. P.; Azevedo, V. Isolation and Characterization of L-Tryptophan Ammonia Lyase from Rubrivivax benzoatilyticus Strain JA2. Curr. Protein Pept. Sci. 2015, 16, 775-781.

(17) Rétey, J. Discovery and Role of Methylidene Imidazolone, a Highly Electrophilic Prosthetic Group. Biochim. Biophys. Acta 2003, 1647, 179-184.

(18) Poppe, L.; Rétey, J. Properties and Synthetic Applications of Ammonia-Lyases. Curr. Org. Chem. 2003, 7, 1297-1315.

(19) Cui, J. D.; Qiu, J. Q.; Fan, X. W.; Jia, S. R.; Tan, Z. L. Biotechnological Production and Applications of Microbial Phenylalanine Ammonia Lyase: A Recent Review. Crit. Rev. Biotechnol. 2013, 34, 258-268.

(20) Kong, J.-Q. Phenylalanine Ammonia-Lyase, a Key Component Used for Phenylpropanoids Production by Metabolic Engineering. RSC Adv. 2015, 5, 6258762603.

(21) Poppe, L.; Paizs, C.; Kovács, K.; Irimie, F. D.; Vertessy, B. G. Preparation of Unnatural Amino Acids with Ammonia-Lyases and 2,3-Aminomutases. In Unnatural Amino Acids: Methods and Protocols; 2012; pp 3-19.

(22) Yamada, T.; Komoto, J.; Takata, Y.; Ogawa, H.; Pitot, H. C.; Takusagawa, F. Crystal Structure of Serine Dehydratase from Rat Liver. Biochemistry 2003, 42, 12854-12865.

(23) Metzler, D. E.; Snell, E. E. Deamination of Serine. II. D-Serine Dehydrase, a Vitamin B 6 Enzyme from Escherichia coli. J. Biol. Chem. 1952, 198, 363-373.

(24) Nishimura, J. S.; Greenberg, D. M. Purification and Properties of L-Threonine Dehydrase of Sheep Liver. J. Biol. Chem. 1961, 236, 2684-2691.

(25) Phillips, A. T.; Wood, W. A. The Mechanism of Action of 5'-Adenylic Acid-Activated Threonine Dehydrase. J. Biol. Chem. 1965, 240, 4703-4709.

(26) Wada, M.; Matsumoto, T.; Nakamori, S.; Sakamoto, M.; Kataoka, M.; Liu, J.-Q.; Itoh, N.; Yamada, H.; Shimizu, S. Purification and Characterization of a Novel Enzyme, Lthreo-3-Hydroxyaspartate Dehydratase, from Pseudomonas sp. T62. FEMS Microbiol. Lett. 1999, 179, 147-151.

(27) Iwamoto, R.; Imanaga, Y.; Soda, K. D-Glucosaminate Dehydratase from Agrobacterium radiobacter. Physicochemical and Enzymological Properties. J. Biochem. 1982, 91, 283289.

(28) Nagasawa, T.; Tanizawa, K.; Satoda, T.; Yamada, H. Diaminopropionate AmmoniaLyase from Salmonella typhimurium. J. Biol. Chem. 1988, 263, 958-964.

(29) Cooper, A. J. L.; Meister, A. Enzymatic Conversion of $O$-Carbamyl-L-Serine to Pyruvate and Ammonia. Biochem. Biophys. Res. Commun. 1973, 55, 780-787.

(30) Iwamoto, R.; Imanga, Y. Enzymatic Microdetermination of D-Glucosaminate with DGlucosaminate Dehydratase. Anal. Lett. 1982, 15, 161-169.

(31) Ito, T.; Takahashi, K.; Naka, T.; Hemmi, H.; Yoshimura, T. Enzymatic Assay of DSerine Using D-Serine Dehydratase from Saccharomyces cerevisiae. Anal. Biochem. 2007, 371, 167-172.

(32) Bradbeer, C. The Clostridial Fermentations of Choline and Ethanolamine. I. Preparation and Properties of Cell-Free Extracts. J. Biol. Chem. 1965, 240, 4669-4674.

(33) Bradbeer, C. The Clostridial Fermentations of Choline and Ethanolamine. II. Requirment for a Cobamide Coenzyme by an Ethanolamine Deaminase. J. Biol. Chem. 1965, 240, 4675-4681. 
(34) Shibata, N.; Tamagaki, H.; Hieda, N.; Akita, K.; Komori, H.; Shomura, Y.; Terawaki, S. I.; Mori, K.; Yasuoka, N.; Higuchi, Y.; Toraya T. Crystal Structures of Ethanolamine Ammonia-Lyase Complexed with Coenzyme $\mathrm{B}_{12}$ Analogs and Substrates. J. Biol. Chem. 2010, 285, 26484-26493.

(35) Toraya, T. Radical Catalysis in Coenzyme $\mathrm{B}_{12}$-Dependent Isomerization (Eliminating) Reactions. Chem. Rev. 2003, 103, 2095-2127.

(36) Toraya, T. Cobalamin-Dependent Dehydratases and a Deaminase: Radical Catalysis and Reactivating Chaperones. Arch. Biochem. Biophys. 2014, 544, 40-57.

(37) Costilow, R. N.; Laycock, L. Ornithine Cyclase (Deaminating). Purification of a Protein That Converts Ornthine to Proline and Definition of the Optimal Assay Conditions. $J$. Biol. Chem. 1971, 246, 6655-6660.

(38) Muth, W. L.; Costilow, R. N. Ornithine Cyclase (Deaminating). III. Mechanism of the Conversion of Ornithine to Proline. J. Biol. Chem. 1974, 249, 7463-7467.

(39) Goodman, J. L.; Wang, S.; Alam, S.; Ruzicka, F. J.; Frey, P. A.; Wedekind, J. E. Ornithine Cyclodeaminase: Structure, Mechanism of Action, and Implications for the $\mu$ Crystallin Family. Biochemistry 2004, 43, 13883-13891.

(40) Khaw, L. E.; Böhm, G. A.; Metcalfe, S.; Staunton, J.; Leadlay, P. F. Mutational Biosynthesis of Novel Rapamycins by a Strain of Streptomyces hygroscopicus NRRL 5491 Disrupted in rapL, Encoding a Putative Lysine Cyclodeaminase. J. Bacteriol. 1998, 180, 809-814.

(41) Gatto, G. J.; Boyne, M. T.; Kelleher, N. L.; Walsh, C. T. Biosynthesis of Pipecolic Acid by RapL, a Lysine Cyclodeaminase Encoded in the Rapamycin Gene Cluster. J. Am. Chem. Soc. 2006, 128, 3838-3847.

(42) Tsotsou, G. E.; Barbirato, F. Biochemical Characterisation of Recombinant Streptomyces pristinaespiralis L-Lysine Cyclodeaminase. Biochimie 2007, 89, 591-604.

(43) Ying, H.; Wang, J.; Wang, Z.; Feng, J.; Chen, K.; Li, Y.; Ouyang, P. Enhanced Conversion of L-Lysine to L-Pipecolic Acid Using a Recombinant Escherichia coli Containing Lysine Cyclodeaminase as Whole-Cell Biocatalyst. J. Mol. Catal. B Enzym. 2015, 117, 75-80.

(44) Jensen, J. V. K.; Wendisch, V. F. Ornithine Cyclodeaminase-Based Proline Production by Corynebacterium glutamicum. Microb. Cell Fact. 2013, 12 (1), 63.

(45) Wu, B.; Szymański, W.; Heberling, M. M.; Feringa, B. L.; Janssen, D. B. Aminomutases: Mechanistic Diversity, Biotechnological Applications and Future Perspectives. Trends Biotechnol. 2011, 29, 352-362.

(46) Frey, P. A.; Reed, G. H. Pyridoxal-5'-phosphate as the Catalyst for Radical Isomerization in Reactions of PLP-Dependent Aminomutases. Biochim. Biophys. Acta 2011, 1814, 1548-1557.

(47) Berkovitch, F.; Behshad, E.; Tang, K.; Enns, E. A.; Frey, P. A.; Drennan, C. L. A Locking Mechanism Preventing Radical Damage in the Absence of Substrate, as Revealed by the X-Ray Structure of Lysine 5,6-Aminomutase. Proc. Natl. Acad. Sci. USA 2004, 101, 15870-15875.

(48) Chen, Y.; Maity, A. N.; Frey, P. A.; Ke, S. Mechanism-Based Inhibition Reveals Transitions Between Two Conformational States in the Action of Lysine 5,6Aminomutase: A Combination of Electron Paramagnetic Resonance Spectroscopy, Electron Nuclear Double Resonance Spectroscopy, and Density Functional Theory Study. J. Am. Chem. Soc. 2013, 135, 788-794. 
(49) Lo, H.; Lin, H.; Maity, A. N.; Ke, S. The Molecular Mechanism of the Open-Closed Protein Conformational Cycle Transitions and Coupled Substrate Binding, Activation and Product Release Events in Lysine 5,6-Aminomutase. Chem. Commun. 2016, 52, 63996402.

(50) Morley, C. G. D.; Stadtman, T. C. Studies on the Fermentation of D- $\alpha$-Lysine. Purification and Properties of an Adenosine Triphosphate Regulated $\mathrm{B}_{12}$-CoenzymeDependent D- $\alpha$-Lysine Mutase Complex from Clostridium sticklandii. Biochemistry 1970, 9, 4890-4900.

(51) Somack, R.; Costilow, R. N. Purification and Properties of a Pyridoxal Phosphate and Coenzyme $\mathrm{B}_{12}$ Dependent D- $\alpha$-Ornithine 5,4-Aminomutase. Biochemistry 1973, 12, 2597-2604.

(52) Chen, H.-P.; Wu, S.-H.; Lin, Y.-L.; Chen, C.-M.; Tsay, S.-S. Cloning, Sequencing, Heterologous Expression, Purification, and Characterization of AdenosylcobalaminDependent D-Ornithine Aminomutase from Clostridium sticklandii. J. Biol. Chem. 2001, 276, 44744-44750.

(53) Chirpich, T. P.; Zappia, V.; Costilow, R. N.; Barker, H. A. Lysine 2,3-Aminomutase: Purification and Properties of a Pyridoxal Phosphate and S-AdenosylmethionineActivated Enzyme. J. Biol. Chem. 1970, 245, 1778-1789.

(54) Song, K. B.; Frey, P. A. Molecular Properties of Lysine-2,3-Aminomutase. J. Biol. Chem. 1991, 266, 7651-7655.

(55) Frey, P. A. Lysine 2,3-Aminomutase: Is Adenosylmethionine a Poor Man's Adenosylcobalamin? FASEB J. 1993, 7, 662-670.

(56) Lepore, B. W.; Ruzicka, F. J.; Frey, P. A.; Ringe, D. The X-Ray Crystal Structure of Lysine-2,3-Aminomutase from Clostridium subterminale. Proc. Natl. Acad. Sci. USA 2005, 102, 13819-13824.

(57) Broderick, J. B.; Duffus, B. R.; Duschene, K. S.; Shepard, E. M. Radical SAdenosylmethionine Enzymes. Chem. Rev. 2014, 114, 4229-4317.

(58) Moss, M.; Frey, P. A. The Role of $S$-Adenosylmethionine in the Lysine 2,3Aminomutase Reaction. J. Biol. Chem. 1987, 262, 14859-14862.

(59) Behshad, E.; Ruzicka, F. J.; Mansoorabadi, S. O.; Chen, D.; Reed, G. H.; Frey, P. A. Enantiomeric Free Radicals and Enzymatic Control of Stereochemistry in a Radical Mechanism: The Case of Lysine 2,3-Aminomutases. Biochemistry 2006, 45, 1263912646.

(60) Ruzicka, F. J.; Frey, P. A. Glutamate 2,3-Aminomutase: A New Member of the Radical SAM Superfamily of Enzymes. Biochim. Biophys. Acta 2007, 1774, 286-296.

(61) Frey, P. A.; Ruzicka, F. J. Glutamate 2,3-Aminomutase and Methods of Use Thereof. US $7456271 \mathrm{~B} 2,2008$.

(62) Liao, H. H.; Gokarn, R. R.; Gort, S. J.; Jessen, H. J.; Selifonova, O. Alanine 2,3Aminomutase. US 7655451 B2, 2010.

(63) Frey, P. A.; Ruzicka, F. J. Methods for the Preparation of $\beta$-Amino Acids. US 7452701 B2, 2008.

(64) Kannangara, C. G.; Gough, S. P. Biosynthesis of $\Delta$-Aminolevulinate in Greening Barley Leaves: Glutamate-1-Semialdehyde Aminotransferase. Carlsberg Res. Commun. 1978, 43, 185-194.

(65) Pugh, C. E.; Harwood, J. L.; John, R. A. Mechanism of Glutamate Semialdehyde Aminotransferase: Roles of Diamino- and Dioxo-Intermediates in the Synthesis of Aminolevulinate. J. Biol. Chem. 1992, 267, 1584-1588. 
(66) Hennig, M.; Grimm, B.; Contestabile, R.; John, R. A.; Jansonius, J. N. Crystal Structure of Glutamate-1-Semialdehyde Aminomutase: An $\alpha_{2}$-Dimeric Vitamin $B_{6}$-Dependent Enzyme with Asymmetry in Structure and Active Site Reactivity. Proc. Natl. Acad. Sci. USA 1997, 94, 4866-4871.

(67) Contestabile, R.; Jenn, T.; Akhtar, M.; Gani, D.; John, R. A. Reactions of Glutamate 1Semialdehyde Aminomutase with $R$ - and $S$-Enantiomers of a Novel, Mechanism-Based Inhibitor, 2,3-Diaminopropyl Sulfate. Biochemistry 2000, 39, 3091-3096.

(68) Campanini, B.; Bettati, S.; Di Salvo, M. L.; Mozzarelli, A.; Contestabile, R. Asymmetry of the Active Site Loop Conformation between Subunits of Glutamate-1-Semialdehyde Aminomutase in Solution. Biomed Res. Int. 2013, 2013, 353270.

(69) Asano, Y.; Kato, Y.; Levy, C.; Baker, P.; Rice, D. Structure and Function of Amino Acid Ammonia-Lyases. Biocatal. Biotransform. 2004, 22, 131-138.

(70) Wu, B.; Szymański, W.; Crismaru, C. G.; Feringa, B. L.; Janssen, D. B. C-N Lyases Catalyzing Addition of Ammonia, Amines and Amides to $\mathrm{C}=\mathrm{C}$ and $\mathrm{C}=\mathrm{O}$ Bonds. In Enzyme Catalysis in Organic Synthesis; 2012; pp 749-778.

(71) Poppe, L. Methylidene-Imidazolone: A Novel Electrophile for Substrate Activation. Curr. Opin. Chem. Biol. 2001, 5, 512-524.

(72) Frey, P. A.; Hegeman, A. D.; Ruzicka, F. J. The Radical SAM Superfamily. Crit. Rev. Biochem. Mol. Biol. 2008, 43, 63-88.

(73) Harden, A. The Chemical Action of Bacillus coli communis and Similar Organisms on Carbohydrates and Allied Compounds. J. Chem. Soc., Trans. 1901, 79, 610-628.

(74) Quastel, J. H.; Woolf, B. The Equilibrium Between L-Aspartic Acid, Fumaric Acid and Ammonia in Presence of Resting Bacteria. Biochem. J. 1926, 20, 545-555.

(75) Rudolph, F. B.; Fromm, H. J. The Purification and Properties of Aspartase from Escherichia coli. Arch. Biochem. Biophys. 1971, 147, 92-98.

(76) Takagi, J. S.; Fukunaga, R.; Tokushige, M.; Katsuki H. Purification, Crystallization, and Molecular Properties of Aspartase from Pseudomonas fluorescens. J. Biochem. 1984, 96, $545-552$.

(77) Wilkinson, J. S.; Williams, V. R. Partial Purification of Bacterial Aspartase by Starch Electrophoresis. Arch. Biochem. Biophys. 1961, 93, 80-84.

(78) Singh, R. S.; Yadav, M. Single-Step Purification and Characterization of Recombinant Aspartase of Aeromonas media NFB-5. Appl. Biochem. Biotechnol. 2012, 167, 991-1001.

(79) Sun, D.; Setlow, P. Cloning, Nucleotide Sequence, and Expression of the Bacillus subtilis ans Operon, Which Codes for L-Asparaginase and L-Aspartase. J. Bacteriol. 1991, 173, 3831-3845.

(80) Kawata, Y.; Tamura, K.; Yano, S.; Mizobata, T.; Nagai, J.; Esaki, N.; Soda, K.; Tokushige, M.; Yumoto, N. Purification and Characterization of Thermostable Aspartase from Bacillus sp. YM55-1. Arch. Biochem. Biophys. 1999, 366, 40-46.

(81) Shi, W.; Dunbar, J.; Jayasekera, M. M. K.; Viola, R. E.; Farber, G. K. The Structure of L -Aspartate Ammonia-Lyase from Escherichia coli. Biochemistry 1997, 36, 9136-9144.

(82) Piatigorsky, J.; O'Brien, W. E.; Norman, B. L.; Kalumuck, K.; Wistow, G. J.; Borras, T.; Nickerson, J. M.; Wawrousek, E. F. Gene Sharing by $\delta$-Crystallin and Argininosuccinate Lyase. Proc. Natl. Acad. Sci. USA 1988, 85, 3479-3483.

(83) Simpson, A.; Bateman, O.; Driessen, H.; Lindley P.; Moss, D.; Mylvaganam, S.; Narebor, E.; Slingsby, C. The Structure of Avian Eye Lens $\delta$-Crystallin Reveals a New Fold for a Superfamily of Oligomeric Enzymes. Nat. Struct. Biol. 1994, 1, 724-734. 
(84) Fibriansah, G.; Veetil, V. P.; Poelarends, G. J.; Thunnissen, A. W. H. Structural Basis for the Catalytic Mechanism of Aspartate Ammonia Lyase. Biochemistry 2011, 50, 60536062.

(85) Falzone, C. J.; Karsten, W. E.; Conley, J. D.; Viola, R. E. L-Aspartase from Escherichia coli: Substrate Specificity and Role of Divalent Metal Ions. Biochemistry 1988, 27, 90899093.

(86) Yoon, M.-Y.; Thayer-Cook, K. A.; Berdis, A. J.; Karsten, W. E.; Schnackerz, K. D.; Cook, P. F. Acid-Base Chemical Mechanism of Aspartase from Hafnia alvei. Arch. Biochem. Biophys. 1995, 320, 115-122.

(87) Porter, D. J. T.; Bright, H. J. 3-Carbanionic Substrate Analogues Bind Very Tightly to Fumarase and Aspartase. J. Biol. Chem. 1980, 255, 4772-4780.

(88) Ellfolk, N. Studies of Aspartase III. On the Specificity of Aspartase. Acta Chem. Scand. 1954, 8, 151-156.

(89) Virtanen, A. I.; Ellfolk, N. Aspartase. Methods Enzymol. 1955, 2, 386-390.

(90) Ma, L.; Yan, B.-X.; Zhao, H.-L.; Liu, W.-H.; You, D.-L.; Cheng, Y.-H. Alteration of Specificity in L-Aspartase in the Presence of Alcohol. Ann. N. Y. Acad. Sci. 1995, 750, 134-137.

(91) Yumoto, N.; Okada, M.; Tokushige, M. Biospecific Inactivation of Aspartase by LAspartic- $\beta$-Semialdehyde. Biochem. Biophys. Res. Commun. 1982, 104, 859-866.

(92) Barker, H. A.; Smyth, R. D.; Wawszkiewicz, E. J.; Lee, M. N.; Wilson, R. M. Enzymic Preparation and Characterization of an $\alpha$-L- $\beta$-Methylaspartic Acid. Arch. Biochem. Biophys. 1958, 78, 468-476.

(93) Barker, H. A.; Smyth, R. D.; Wilson, R. M.; Weissbach, H. The Purification and Properties of $\beta$-Methylaspartase. J. Biol. Chem. 1958, 234, 320-328.

(94) Asano, Y.; Kato, Y. Occurrence of 3-Methylaspartate Ammonia-Lyase in Facultative Anaerobes and Their Application to Synthesis of 3-Substituted (S)-Aspartic Acids. Biosci. Biotechnol. Biochem. 1994, 58, 223-224.

(95) Kato, Y.; Asano, Y. Purification and Properties of Crystalline 3-Methylaspartase from Two Facultative Anaerobes, Citrobacter sp. Strain YG-0504 and Morganella morganii strain YG-0601. Biosci. Biotech. Biochem. 1995, 59, 93-99.

(96) Archer, C. H.; Thomas, N. R.; Gani, D. Synthesis of $(2 S, 3 R)-$ and $(2 S, 3 R)\left[3-{ }^{2} \mathrm{H}\right]-3-$ Methylaspartic Acid: Slow Substrates for a syn-Elimination Reaction Catalysed by Methylaspartase. Tetrahedron: Asymmetry 1993, 4 (6), 1141-1152.

(97) Goda, S. K.; Minton, N. P.; Botting, N. P.; Gani, D. Cloning, Sequencing, and Expression in Escherichia coli of the Clostridium tetanomorphum Gene Encoding $\beta$-Methylaspartase and Characterization of the Recombinant Protein. Biochemistry 1992, 31, 10747-10756.

(98) Kato, Y.; Asano, Y. Cloning, Nucleotide Sequencing, and Expression of the 3Methylaspartate Ammonia-Lyase Gene from Citrobacter amalonaticus Strain YG-1002. Appl. Microbiol. Biotechnol. 1998, 50, 468-474.

(99) Bearne, S. L.; White, R. L.; MacDonnell, J. E.; Bahrami, S.; Grønlund, J. Purification and Characterization of $\beta$-Methylaspartase from Fusobacterium varium. Mol. Cell. Biochem. 2001, 221, 117-126.

(100) Raj, H.; Veetil, V. P.; Szymanski, W.; Dekker, F. J.; Quax, W. J.; Feringa, B. L.; Janssen, D. B.; Poelarends, G. J. Characterization of a Thermostable Methylaspartate Ammonia Lyase from Carboxydothermus hydrogenoformans. Appl. Microbiol. Biotechnol. 2012, 94, 385-397. 
(101) Gerlt, J. A.; Babbitt, P. C. The Enolase Superfamily: Different Reactions Catalyzed by Similar Active Sites. FASEB J. 1997, 11, A1006.

(102) Bright, H. J.; Ingraham, L. L.; Lundin, R. E. The Mechanism of the Methylaspartate Ammonia-Lyase Reaction: Deuterium Exchange. Biochim. Biophys. Acta 1964, 81, 576584.

(103) Raj, H.; Weiner, B.; Veetil, V. P.; Reis, C. R.; Quax, W. J.; Janssen, D. B.; Feringa, B. L.; Poelarends, G. J. Alteration of the Diastereoselectivity of 3-Methylaspartate Ammonia Lyase by Using Structure-Based Mutagenesis. ChemBioChem 2009, 10, 2236-2245.

(104) Raj, H.; Poelarends, G. J. The Roles of Active Site Residues in the Catalytic Mechanism of Methylaspartate Ammonia-Lyase. FEBS Open Bio 2013, 3, 285-290.

(105) Emiliani, G.; Fondi, M.; Fani, R.; Gribaldo, S. A Horizontal Gene Transfer at the Origin of Phenylpropanoid Metabolism: A Key Adaptation of Plants to Land. Biol. Direct 2009, 4,7 .

(106) Zhang, X.; Liu, C.-J. Multifaceted Regulations of Gateway Enzyme Phenylalanine Ammonia-Lyase in the Biosynthesis of Phenylpropanoids. Mol. Plant 2015, 8, 17-27.

(107) Watts, K. T.; Mijts, B. N.; Lee, P. C.; Manning, A. J.; Schmidt-Dannert, C. Discovery of a Substrate Selectivity Switch in Tyrosine Ammonia-Lyase, a Member of the Aromatic Amino Acid Lyase Family. Chem. Biol. 2006, 13, 1317-1326.

(108) Hodgins, D. S. Yeast Phenylalanine Ammonia-Lyase: Purification, Properties, and the Identification of Catalytically Essential Dehydroalanine. J. Biol. Chem. 1971, 246, 29772985.

(109) Calabrese, J. C.; Jordan, D. B.; Boodhoo, A.; Sariaslani, S.; Vannelli, T. Crystal Structure of Phenylalanine Ammonia Lyase: Multiple Helix Dipoles Implicated in Catalysis. Biochemistry 2004, 43, 11403-11416.

(110) Ritter, H.; Schulz, G. E. Structural Basis for the Entrance into the Phenylpropanoid Metabolism Catalyzed by Phenylalanine Ammonia-Lyase. Plant Cell 2004, 16, 34263436.

(111) Baedeker, M.; Schulz, G. E. Overexpression of a Designed $2.2 \mathrm{~Kb}$ Gene of Eukaryotic Phenylalanine Ammonia-Lyase in Escherichia Coli. FEBS Lett. 1999, 457, 57-60.

(112) Moffitt, M. C.; Louie, G. V; Bowman, M. E.; Pence, J.; Noel, J. P.; Joseph, P.; Moore, B. S. Discovery of Two Cyanobacterial Phenylalanine Ammonia Lyases: Kinetic and Structural Characterization. Biochemistry 2007, 46, 1004-1012.

(113) Zhu, Y.; Liao, S.; Ye, J.; Zhang, H. Cloning and Characterization of a Novel Tyrosine Ammonia Lyase-Encoding Gene Involved in Bagremycins Biosynthesis in Streptomyces sp. Biotechnol. Lett. 2012, 34, 269-274.

(114) Peterkofsky, A. The Mechanism of Action of Histidase: Amino-Enzyme Formation and Partial Reactions. J. Biol. Chem. 1962, 237, 787-795.

(115) Consevage, M. W.; Phillips, A. T. Presence and Quantity of Dehydroalanine in Histidine Ammonia-Lyase from Pseudomonas putida. Biochemistry 1985, 24, 301-308.

(116) Smith, T.A.; Cordelle, F. H.; Abeles R. H. Inactivation of Histidine Deaminase by Carbonyl Reagents. Arch. Biochem. Biophys. 1967, 120, 724-725.

(117) Givot, I. L.; Smith, T. A.; Abeles, R. H. Studies on the Mechanism and the Structure of the Electrophilic Center of Histidine Ammonia Lyase. J. Biol. Chem. 1969, 244, 63416353.

(118) Hanson, K. R.; Havir, E. A. L-Phenylalanine Ammonia-Lyase. IV. Evidence That the Prosthetic Group Contains a Dehydroalanyl Residue and Mechanism of Action. Arch. Biochem. Biophys. 1970, 141, 1-17. 
(119) Langer, M.; Reck, G.; Reed, J.; Rétey, J. Identification of Serine-143 as the Most Likely Precursor of Dehydroalanine in the Active Site of Histidine Ammonia-Lyase. A Study of the Overexpressed Enzyme by Site-Directed Mutagenesis. Biochemistry 1994, 33, 64626467.

(120) Schuster, B.; Retey, J. Serine-202 Is the Putative Precursor of the Active Site Dehydroalanine of Phenylalanine Ammonia Lyase. Site-Directed Mutagenesis Studies on the Enzyme from Parsley (Petroselinum crispum L.). FEBS Lett. 1994, 349, 252-254.

(121) Schroeder, A. C.; Kumaran, S.; Hicks, L. M.; Cahoon, R. E.; Halls, C.; Yu, O.; Jez, J. M. Contributions of Conserved Serine and Tyrosine Residues to Catalysis, Ligand Binding, and Cofactor Processing in the Active Site of Tyrosine Ammonia Lyase. Phytochemistry 2008, 69, 1496-1506.

(122) Langer, B.; Röther, D.; Rétey, J. Identification of Essential Amino Acids in Phenylalanine Ammonia-Lyase by Site-Directed Mutagenesis. Biochemistry 1997, 36, 10867-10871.

(123) Langer, M.; Lieber, A.; Rétey, J. Histidine Ammonia-Lyase Mutant S143C Is Posttranslationally Converted into Fully Active Wild-Type Enzyme. Evidence for Serine 143 to Be the Precursor of Active Site Dehydroalanine. Biochemistry 1994, 33, 1403414038.

(124) Schwede, T. F.; Rétey, J.; Schulz, G. E. Crystal Structure of Histidine Ammonia-Lyase Revealing a Novel Polypeptide Modification as the Catalytic Electrophile. Biochemistry 1999, 38, 5355-5361.

(125) Ormö, M.; Cubitt, A. B.; Kallio, K.; Gross, L. A.; Tsien, R. Y.; Remington, S. J. Crystal Structure of the Aequorea victoria Green Fluorescent Protein. Science 1996, 273, 13921395.

(126) Reid, B. G.; Flynn, G. C. Chromophore Formation in Green Fluorescent Protein. Biochemistry 1997, 36, 6786-6791.

(127) Sánchez-Murcia, P. A.; Bueren-Calabuig, J. A.; Camacho-Artacho, M.; Cortés-Cabrera, Á.; Gago, F. Stepwise Simulation of 3,5-Dihydro-5-Methylidene-4H-Imidazol-4-One (MIO) Biogenesis in Histidine Ammonia-Lyase. Biochemistry 2016, 55, 5854-5864.

(128) Havir, E. A.; Hanson, K. R. L-Phenylalanine Ammonia-Lyase (Maize and Potato). Evidence That the Enzyme Is Composed of Four Subunits. Biochemistry 1973, 12, 15831591.

(129) Pilbák, S.; Tomin, A.; Rétey, J.; Poppe, L. The Essential Tyrosine-Containing Loop Conformation and the Role of the $C$-Terminal Multi-Helix Region in Eukaryotic Phenylalanine Ammonia-Lyases. FEBS J. 2006, 273, 1004-1019.

(130) Wang, L.; Gamez, A.; Archer, H.; Abola, E. E.; Sarkissian, C. N.; Fitzpatrick, P.; Wendt, D.; Zhang, Y.; Vellard, M.; Bliesath, J.; Bell, S. M.; Lemontt, J. F.; Scriver, C. R.; Stevens, R. C. Structural and Biochemical Characterization of the Therapeutic Anabaena variabilis Phenylalanine Ammonia Lyase. J. Mol. Biol. 2008, 380, 623-635.

(131) Röther, D.; Merkel, D.; Rétey, J. Spectroscopic Evidence for a 4-Methylidene Imidazol5-One in Histidine and Phenylalanine Ammonia-Lyases. Angew. Chem. Int. Ed. 2000, 39, $2462-2464$.

(132) Louie, G. V; Bowman, M. E.; Moffitt, M. C.; Baiga, T. J.; Moore, B. S.; Noel, J. P. Structural Determinants and Modulation of Substrate Specificity in PhenylalanineTyrosine Ammonia-Lyases. Chem. Biol. 2006, 13, 1327-1338. 
(133) Röther, D.; Poppe, L.; Morlock, G.; Viergutz, S.; Rétey, J. An Active Site Homology Model of Phenylalanine Ammonia-Lyase from Petroselinum crispum. Eur. J. Biochem. 2002, 269, 3065-3075.

(134) Krug, D.; Müller, R. Discovery of Additional Members of the Tyrosine Aminomutase Enzyme Family and the Mutational Analysis of CmdF. ChemBioChem 2009, 10, 741750.

(135) Rösler, J.; Krekel, F.; Amrhein, N.; Schmid, J. Maize Phenylalanine Ammonia-Lyase Has Tyrosine Ammonia-Lyase Activity. Plant Physiol. 1997, 113, 175-179.

(136) Klee, C. B. Metal Activation of Histidine Ammonia-Lyase. Metal Ion-SulfhydrylGroup Relationship. J. Biol. Chem. 1972, 247, 1398-1406.

(137) Seff, A.-L.; Pilbák, S.; Silaghi-Dumitrescu, I.; Poppe, L. Computational Investigation of the Histidine Ammonia-Lyase Reaction : A Modified Loop Conformation and the Role of the Zinc(II) Ion. J. Mol. Model. 2011, 17, 1551-1563.

(138) Röther, D.; Poppe, L.; Viergutz, S.; Langer, B.; Rétey, J. Characterization of the Active Site of Histidine Ammonia-Lyase from Pseudomonas putida. Eur. J. Biochem. 2001, 268, 6011-6019.

(139) Bartsch, S.; Bornscheuer, U. T. Mutational Analysis of Phenylalanine Ammonia Lyase to Improve Reactions Rates for Various Substrates. Protein Eng. Des. Sel. 2010, 23, 929933.

(140) Hermes, J. D.; Weiss, P. M.; Cleland, W. W. Use of Nitrogen-15 and Deuterium Isotope Effects to Determine the Chemical Mechanism of Phenylalanine Ammonia-Lyase. Biochemistry 1985, 24, 2959-2967.

(141) Pilbák, S.; Farkas, Ö.; Poppe, L. Mechanism of the Tyrosine Ammonia Lyase Reaction. Tandem Nucleophilic and Electrophilic Enhancement by a Proton Transfer. Chem. Eur. J. 2012, 18, 7793-7802.

(142) Langer, M.; Pauling, A.; Rétey, J. The Role of Dehydroalanine in Catalysis by Histidine Ammonia Lyase. Angew. Chem. Int. Ed. 1995, 34, 1464-1465.

(143) Schuster, B.; Rétey, J. The Mechanism of Action of Phenylalanine Ammonia-Lyase: The Role of Prosthetic Dehydroalanine. Proc. Natl. Acad. Sci. USA 1995, 92, 8433-8437.

(144) Poppe, L.; Rétey, J. Friedel-Crafts-Type Mechanism for the Enzymatic Elimination of Ammonia from Histidine and Phenylalanine. Angew. Chem. Int. Ed. 2005, 44, 36683688 .

(145) Baedeker, M.; Schulz, G. E. Structures of Two Histidine Ammonia-Lyase Modifications and Implications for the Catalytic Mechanism. Eur. J. Biochem. 2002, 269, 1790-1797.

(146) Rettig, M.; Sigrist, A.; Rétey, J. Mimicking the Reaction of Phenylalanine Ammonia Lyase by a Synthetic Model. Helv. Chim. Acta 2000, 83, 2246-2265.

(147) Pinto, G. P.; Ribeiro, A. J. M.; Ramos, M. J.; Fernandes, P. A.; Toscano, M.; Russo, N. New Insights in the Catalytic Mechanism of Tyrosine Ammonia-Lyase given by QM/MM and QM Cluster Models. Arch. Biochem. Biophys. 2015, 582, 107-115.

(148) Strange, P. G.; Staunton, J.; Wiltshire, H. R.; Battersby, A. R.; Hanson, K. R.; Havir, E. A. Studies of Enzyme-Mediated Reactions. Part II. Stereochemistry of the Elimination of Ammonia from L-Tyrosine Catalysed by the Enzyme Form Maize. J. Chem. Soc. Perkin Trans. 1 1972, 2364-2372.

(149) Weiser, D.; Bencze, L. C.; Banóczi, G.; Ender, F.; Kiss, R.; Kokai, E.; Szilágyi, A.; Vértessy, B. G.; Farkas, Ö.; Paizs, C.; Poppe, L. Phenylalanine Ammonia-LyaseCatalyzed Deamination of an Acyclic Amino Acid: Enzyme Mechanistic Studies Aided 
by a Novel Microreactor Filled with Magnetic Nanoparticles. ChemBioChem 2015, 16, 2283-2288.

(150) Zoń, J.; Amrhein, N. Inhibitors of Phenylalanine Ammonia-Lyase: 2-Aminoindan-2Phosphonic Acid and Related Compounds. Liebigs Ann. Chem. 1992, 625-628.

(151) Gloge, A.; Zoń, J.; Kövári, Á.; Poppe, L.; Rétey, J. Phenylalanine Ammonia-Lyase: The Use of Its Broad Substrate Specificity for Mechanistic Investigations and Biocatalysis. Synthesis of L-Arylalanines. Chem. Eur. J. 2000, 6, 3386-3390.

(152) Klee, C. B.; Kirk, K. L.; Cohen, L. A. 4-Nitro-L-Histidine as a Substrate for Histidine Ammonia-Lyase: The Role of $\beta$-Hydrogen Acidity in the Rate-Limiting Step. Biochem. Biophys. Res. Commun. 1979, 87, 343-348.

(153) Toşa, M. I.; Brem, J.; Mantu, A.; Irimie, F. D.; Paizs, C.; Rétey, J. The Interaction of Nitrophenylalanines with Wild Type and Mutant 4-Methylideneimidazole-5-One-Less Phenylalanine Ammonia Lyase. ChemCatChem 2013, 5, 779-783.

(154) Lovelock, S. L.; Lloyd, R. C.; Turner, N. J. Phenylalanine Ammonia Lyase Catalyzed Synthesis of Amino Acids by an MIO-Cofactor Independent Pathway. Angew. Chem. Int. Ed. 2014, 53, 4652-4656.

(155) Parmeggiani, F.; Lovelock, S. L.; Weise, N. J.; Ahmed, S. T.; Turner, N. J. Synthesis of D- and L-Phenylalanine Derivatives by Phenylalanine Ammonia Lyases: A Multienzymatic Cascade Process. Angew. Chem. Int. Ed. 2015, 54, 4608-4611.

(156) Christenson, S. D.; Liu, W.; Toney, M. D.; Shen, B. A Novel 4-Methylideneimidazole-5One-Containing Tyrosine Aminomutase in Enediyne Antitumor Antibiotic C-1027 Biosynthesis. J. Am. Chem. Soc. 2003, 125, 6062-6063.

(157) Christenson, S. D.; Wu, W.; Spies, M. A.; Shen, B.; Toney, M. D. Kinetic Analysis of the 4-Methylideneimidazole-5-One-Containing Tyrosine Aminomutase in Enediyne Antitumor Antibiotic C-1027 Biosynthesis. Biochemistry 2003, 42, 12708-12718.

(158) Christianson, C. V; Montavon, T. J.; Van Lanen, S. G.; Shen, B.; Bruner, S. D. The Structure of L-Tyrosine 2,3-Aminomutase from the C-1027 Enediyne Antitumor Antibiotic Biosynthetic Pathway. Biochemistry 2007, 46, 7205-7214.

(159) Van Lanen, S. G.; Oh, T.; Liu, W.; Wendt-Pienkowski, E.; Shen, B.; Madison, W. Characterization of the Maduropeptin Biosynthetic Gene Cluster from Actinomadura madurae ATCC 39144 Supporting a Unifying Paradigm for Enediyne Biosynthesis. $J$. Am. Chem. Soc. 2007, 129, 13082-13094.

(160) Huang, S.-X.; Lohman, J. R.; Huang, T.; Shen, B. A New Member of the 4Methylideneimidazole-5-One-Containing Aminomutase Family from the Enediyne Kedarcidin Biosynthetic Pathway. Proc. Natl. Acad. Sci. USA 2013, 110, 8069-8074.

(161) Rachid, S.; Krug, D.; Kunze, B.; Kochems, I.; Scharfe, M.; Zabriskie, T. M.; Blöcker, H.; Müller, R. Molecular and Biochemical Studies of Chondramide Formation. Highly Cytotoxic Natural Products from Chondromyces crocatus $\mathrm{Cm}$ c5. Chem. Biol. 2006, 13, 667-681.

(162) Rachid, S.; Krug, D.; Weissman, K. J.; Müller, R. Biosynthesis of (R)- $\beta$-Tyrosine and Its Incorporation into the Highly Cytotoxic Chondramides Produced by Chondromyces crocatus. J. Biol. Chem. 2007, 282, 21810-21817.

(163) Wanninayake, U.; Walker, K. D. A Bacterial Tyrosine Aminomutase Proceeds through Retention or Inversion of Stereochemistry to Catalyze Its Isomerization Reaction. J. Am. Chem. Soc. 2013, 135, 11193-11204.

(164) Spiteller, P.; Rüth, M.; Von Nussbaum, F.; Steglich, W. Detection of a 2,3-Aminomutase in the Mushroom Cortinarius violaceus. Angew. Chem. Int. Ed. 2000, 39, 2754-2756. 
(165) Yan, J.; Aboshi, T.; Teraishi, M.; Strickler, S. R.; Spindel, J. E.; Tung, C.-W.; Takata, R.; Matsumoto, F.; Maesaka, Y.; McCouch, S. R.; Okumoto, Y.; Mori, N.; Jander, G. The Tyrosine Aminomutase TAM1 Is Required for $\beta$-Tyrosine Biosynthesis in Rice. Plant Cell 2015, 27, 1265-1278.

(166) Walter, T.; King, Z.; Walker, K. D. A Tyrosine Aminomutase from Rice (Oryza sativa) Isomerizes (S)- $\alpha$ - to $(\mathrm{R})-\beta$-Tyrosine with Unique High Enantioselectivity and Retention of Configuration. Biochemistry 2016, 55, 1-4.

(167) Walter, T.; Wijewardena, D.; Walker, K. D. Mutation of Aryl Binding Pocket Residues Results in an Unexpected Activity Switch in an Oryza sativa Tyrosine Aminomutase. Biochemistry 2016, 55, 3497-3503.

(168) Bartsch, S.; Bornscheuer, U. T. A Single Residue Influences the Reaction Mechanism of Ammonia Lyases and Mutases. Angew. Chem. Int. Ed. 2009, 48, 3362-3365.

(169) Walker, K. D.; Klettke, K.; Akiyama, T.; Croteau, R. Cloning, Heterologous Expression, and Characterization of a Phenylalanine Aminomutase Involved in Taxol Biosynthesis. $J$. Biol. Chem. 2004, 279, 53947-53954.

(170) Feng, L.; Wanninayake, U.; Strom, S.; Geiger, J.; Walker, K. D. Mechanistic, Mutational, and Structural Evaluation of a Taxus Phenylalanine Aminomutase. Biochemistry 2011, 50, 2919-2930.

(171) Steele, C. L.; Chen, Y.; Dougherty, B. A.; Li, W.; Hofstead, S.; Lam, K. S.; Xing, Z.; Chiang, S.-J. Purification, Cloning, and Functional Expression of Phenylalanine Aminomutase: The First Committed Step in Taxol Side-Chain Biosynthesis. Arch. Biochem. Biophys. 2005, 438, 1-10.

(172) Amos, L. A.; Löwe, J. How Taxol ${ }^{\circledR}$ Stabilises Microtubule Structure. Chem. Biol. 1999, 6, R65-R69.

(173) Roopa, G.; Madhusudhan, M. C.; Sunil, K. C. R.; Lisa, N.; Calvin, R.; Poornima, R.; Zeinab, N.; Kini, K. R.; Prakash, H. S.; Geetha, N. Identification of Taxol-Producing Endophytic Fungi Isolated from Salacia oblonga through Genomic Mining Approach. $J$. Genet. Eng. Biotechnol. 2015, 13, 119-127.

(174) Walker, K. D.; Floss, H. G. Detection of a Phenylalanine Aminomutase in Cell-Free Extracts of Taxus brevifolia and Preliminary Characterization of Its Reaction. J. Am. Chem. Soc. 1998, 120, 5333-5334.

(175) Mutatu, W.; Klettke, K. L.; Foster, C.; Walker, K. D. Unusual Mechanism for an Aminomutase Rearrangement: Retention of Configuration at the Migration Termini. Biochemistry 2007, 46, 9785-9794.

(176) Wybenga, G. G.; Szymański, W.; Wu, B.; Feringa, B. L.; Janssen, D. B.; Dijkstra, B. W. Structural Investigations into the Stereochemistry and Activity of a Phenylalanine-2,3Aminomutase from Taxus chinensis. Biochemistry 2014, 53, 3187-3198.

(177) Ratnayake, N. D.; Wanninayake, U.; Geiger, J. H.; Walker, K. D. Stereochemistry and Mechanism of a Microbial Phenylalanine Aminomutase. J. Am. Chem. Soc. 2011, 133, 8531-8533.

(178) Wu, B.; Szymański, W.; Wybenga, G. G.; Heberling, M. M.; Bartsch, S.; De Wildeman, S.; Poelarends, G. J.; Feringa, B. L.; Dijkstra, B. W.; Janssen, D. B. Mechanism-Inspired Engineering of Phenylalanine Aminomutase for Enhanced $\beta$-Regioselective Asymmetric Amination of Cinnamates. Angew. Chem. Int. Ed. 2012, 51, 482-486.

(179) Wang, K.; Hou, Q.; Liu, Y. Insight into the Mechanism of Aminomutase Reaction: A Case Study of Phenylalanine Aminomutase by Computational Approach. J. Mol. Graph. Model. 2013, 46, 65-73. 
(180) Bartsch, S.; Wybenga, G. G.; Jansen, M.; Heberling, M. M.; Wu, B.; Dijkstra, B. W.; Janssen, D. B. Redesign of a Phenylalanine Aminomutase into a Phenylalanine Ammonia Lyase. ChemCatChem 2013, 5, 1797-1802.

(181) Ghosh, A. C.; Manmade, A.; Townsend, J. M.; Bousquet, A.; Howes, J. F.; Demain, A. L. Production of Cyclochlorotine and a New Metabolite, Simatoxin, by Penicillium islandicum Sopp. Appl. Environ. Microbiol. 1978, 35, 1074-1078.

(182) Morita, H.; Nagashima, S.; Takeya, K.; Itokawa, H.; Iitaka, Y. Structures and Conformation of Antitumour Cyclic Pentapeptides, Astins A, B and C, from Aster tataricus. Tetrahedron 1995, 51, 1121-1132.

(183) Schafhauser, T.; Wibberg, D.; Rueckert, C.; Winkler, A.; Flor, L.; Van Pée, K.-H.; Fewer, D. P.; Sivonen, K.; Jahn, L.; Ludwig-Müller, J.; Caradec, T.; Jacques, P.; Huijbers, M. M. E.; Van Berkel, W. J. H.; Weber, T.; Wohlleben, W.; Kalinowski, J. Draft Genome Sequence of Talaromyces islandicus ("Penicillium islandicum") WF-3812, a Neglected Mold with Significant Biotechnological Potential. J. Biotechnol. 2015, $211,101-102$.

(184) Schafhauser, T.; Kirchner, N.; Kulik, A.; Huijbers, M. M. E.; Flor, L.; Caradec, T.; Fewer, D. P.; Gross, H.; Jacques, P.; Jahn, L.; Jokela, J.; Leclere, V.; Ludwig-Muller, J.; Sivonen, K.; Van Berkel, W. J. H.; Weber, T.; Wohlleben, W.; Van Pée, K.-H. The Cyclochlorotine Mycotoxin Is Produced by the Nonribosomal Peptide Synthetase CctN in Talaromyces islandicus ("Penicillium islandicum"). Environ. Microbiol. 2016, 18, 37283741.

(185) Jahn, L. Characterization of a New Endophytic Astin Producer, Pelliciarosea asterica, from Aster tataricus, TU Dresden, 2015.

(186) Jin, M.; Fischbach, M. A.; Clardy, J. A Biosynthetic Gene Cluster for the Acetyl-CoA Carboxylase Inhibitor Andrimid. J. Am. Chem. Soc. 2006, 128, 10660-10661.

(187) Magarvey, N. A.; Fortin, P. D.; Thomas, P. M.; Kelleher, N. L.; Walsh, C. T. Gatekeeping versus Promiscuity in the Early Stages of the Andrimid Biosynthetic Assembly Line. ACS Chem. Biol. 2008, 3, 542-554.

(188) Strom, S.; Wanninayake, U.; Ratnayake, N. D.; Walker, K. D.; Geiger, J. H. Insights into the Mechanistic Pathway of the Pantoea agglomerans Phenylalanine Aminomutase. Angew. Chem. Int. Ed. 2012, 51 (12), 2898-2902.

(189) Weise, N. J.; Ahmed, S. T.; Parmeggiani, F.; Turner, N. J. Kinetic Resolution of Aromatic $\beta$-Amino Acids Using a Combination of Phenylalanine Ammonia Lyase and Aminomutase Biocatalysts. Adv. Synth. Catal. 2017, in press, DOI:10.1002/adsc.201600894.

(190) Kudo, F.; Kawamura, K.; Uchino, A.; Miyanaga, A.; Numakura, M.; Takayanagi, R.; Eguchi, T. Genome Mining of the Hitachimycin Biosynthetic Gene Cluster: Involvement of a Phenylalanine-2,3-Aminomutase in Biosynthesis. ChemBioChem 2015, 16, 909-914.

(191) Xiang, L.; Moore, B. S. Inactivation, Complementation, and Heterologous Expression of encP, a Novel Bacterial Phenylalanine Ammonia-Lyase Gene. J. Biol. Chem. 2002, 277, 32505-32509.

(192) Xiang, L.; Moore, B. S. Biochemical Characterization of a Prokaryotic Phenylalanine Ammonia Lyase. J. Bacteriol. 2005, 187, 4286-4289.

(193) Chesters, C.; Wilding, M.; Goodall, M.; Micklefield, J. Thermal Bifunctionality of Bacterial Phenylalanine Aminomutase and Ammonia Lyase Enzymes. Angew. Chem. Int. Ed. 2012, 51, 4344-4348. 
(194) Tosa, T.; Sato, T.; Mori, T.; Chibata, I. Basic Studies for Continuous Production of LAspartic Acid by Immobilized Escherichia coli Cells. Appl. Microbiol. 1974, 27, 886889.

(195) Fusee, M. C. Industrial Production of L-Aspartic Acid Using Polyurethane-Immobilized Cells Containing Aspartase. Methods Enzymol. 1987, 136, 463-471.

(196) Nishida, Y.; Sato, T.; Tosa, T.; Chibata, I. Immobilization of Escherichia coli Cells Having Aspartase Activity with Carrageenan and Locust Bean Gum. Enzym. Microb. Technol. 1979, 1, 95-99.

(197) Chibata, I.; Tosa, T.; Sato, T. Continuous Production of L-Aspartic Acid. Improvement of Productivity by Both Development of Immobilization Method and Construction of New Escherichia coli Strain. Appl. Biochem. Biotechnol. 1986, 13, 231-240.

(198) Kimura, K.; Takayama, K.; Ado, Y.; Kawamoto, T.; Masunaga, I. Process for Producing Thermophilic Aspartase. US 4391910, 1983.

(199) Röhm, K. H.; Van Etten, R. L. Stereospecific Synthesis of L-[1,4- $\left.{ }^{13} \mathrm{C}_{2}\right]$ Aspartic Acid, L$\beta-\left(\left[{ }^{13} \mathrm{C}\right]\right.$ Cyano)alanine and L-[4- $\left.{ }^{13} \mathrm{C}\right]$ Aspartic Acid. J. Labelled Compd. Radiopharm. 1985, 22, 909-915.

(200) Tajima, T.; Hamada, M.; Nakashimada, Y.; Kato, J. Efficient Aspartic Acid Production by a Psychrophile-Based Simple Biocatalyst. J. Ind. Microbiol. Biotechnol. 2015, 42, 1319-1324.

(201) Sherwin, M. B.; Blouin, J. J. Process for Preparing L-Aspartic Acid. US 4560653, 1985.

(202) Sakano, K.; Hayashi, T.; Mukouyama, M. Process for Production of L-Aspartic Acid. US 5541090, 1996.

(203) Cárdenas-Fernández, M.; Khalikova, E.; Korpela, T.; López, C.; Álvaro, G. CoImmobilised Aspartase and Transaminase for High-Yield Synthesis of L-Phenylalanine. Biochem. Eng. J. 2015, 93, 173-178.

(204) Han, C.; Yao, P.; Yuan, J.; Duan, Y.; Feng, J.; Wang, M.; Wu, Q.; Zhu, D. NitrilaseCatalyzed Hydrolysis of 3-Aminopropionitrile at High Concentration with a Tandem Reaction Strategy for Shifting the Reaction to $\beta$-Alanine Formation. J. Mol. Catal. B Enzym. 2015, 115, 113-118.

(205) Tessaro, D.; Pollegioni, L.; Piubelli, L.; D’Arrigo, P.; Servi, S. Systems Biocatalysis: An Artificial Metabolism for Interconversion of Functional Groups. ACS Catal. 2015, 5, 1604-1608.

(206) Emery, T. F. Aspartase-Catalyzed Synthesis of N-Hydroxyaspartic Acid. Biochemistry 1963, 2, 1041-1045.

(207) Weiner, B.; Poelarends, G. J.; Janssen, D. B.; Feringa, B. L. Biocatalytic Enantioselective Synthesis of $N$-Substituted Aspartic Acids by Aspartate Ammonia Lyase. Chem. Eur. J. 2008, 14, 10094-10100.

(208) Vogel, A.; Schmiedel, R.; Hofmann, U.; Gruber, K.; Zangger, K. Converting Aspartase into a $\beta$-Amino Acid Lyase by Cluster Screening. ChemCatChem 2014, 6, 965-968.

(209) Winkler, M. F.; Williams, V. R. New Substrates for $\beta$-Methylaspartase. Biochim. Biophys. Acta 1967, 146, 287-289.

(210) Akhtar, M.; Cohen, M. A.; Gani, D. Enzymic Synthesis of 3-Halogenoaspartic Acids Using $\beta$-Methylaspartase: Inhibition by 3-Bromoaspartic Acid. J. Chem. Soc., Chem. Commun. 1986, 1290-1291.

(211) Akhtar, M.; Cohen, M. A.; Gani, D. Stereochemical Course of the Enzymic Amination of Chloro- and Bromo- Fumaric Acid by 3-Methylaspartate Ammonia-Lyase. Tetrahedron Lett. 1987, 28 (21), 2413-2416. 
(212) Akhtar, M.; Botting, N. P.; Cohen, M. A.; Gani, D. Enantiospecific Synthesis of 3Substituted Aspartic Acids via Enzymic Amination of Substituted Fumaric Acids. Tetrahedron 1987, 43, 5899-5908.

(213) Gulzar, M. S.; Akhtar, M.; Gani, D. Preparation of $N$-Substituted Aspartic Acids via Enantiospecific Conjugate Addition of $\mathrm{N}$-Nucleophiles to Fumaric Acids Using Methylaspartase: Synthetic Utility and Mechanistic Implications. J. Chem. Soc. Perkin Trans. 1 1997, 649-655.

(214) Raj, H.; Szymański, W.; De Villiers, J.; Rozeboom, H. J.; Veetil, V. P.; Reis, C. R.; De Villiers, M.; Dekker, F. J.; De Wildeman, S.; Quax, W. J.; Thunnissen, A. W. H.; Feringa, B. L.; Janssen, D. B.; Poelarends, G. J. Engineering Methylaspartate Ammonia Lyase for the Asymmetric Synthesis of Unnatural Amino Acids. Nat. Chem. 2012, 4, 478-484.

(215) Song, C. W.; Lee, J.; Ko, Y.-S.; Lee, S. Y. Metabolic Engineering of Escherichia coli for the Production of 3-Aminopropionic Acid. Metab. Eng. 2015, 30, 121-129.

(216) Wang, J.; Zhang, K. Production of Mesaconate in Escherichia coli by Engineered Glutamate Mutase Pathway. Metab. Eng. 2015, 30, 190-196.

(217) Williams, V. R.; Hiroms, J. M. Reversibility of the "Irreversible" Histidine AmmoniaLyase Reaction. Biochim. Biophys. Acta 1967, 139, 214-216.

(218) Havir, E. A.; Hanson, K. R. L-Phenylalanine Ammonia-Lyase. II. Mechanism and Kinetic Properties of the Enzyme from Potato Tubers. Biochemistry 1968, 7, 1904-1914.

(222) Nelson, R. P. Immobilized Microbial Cells. US 3957580, 1976.

(223) Yamada, S.; Nabe, K.; Izuo, N.; Nakamichi, K.; Chibata, I. Production of LPhenylalanine from trans-Cinnamic Acid with Rhodotorula glutinis Containing LPhenylalanine Ammonia-Lyase Activity. Appl. Environ. Microbiol. 1981, 42, 773-778.

(224) Renard, G.; Guilleux, J.-C.; Bore, C.; Malta-Valette, V.; Lerner, D. A. Synthesis of LPhenylalanine Analogs by Rhodotorula glutinis. Bioconversion of Cinnamic Acid Derivatives. Biotechnol. Lett. 1992, 14, 673-678.

(225) Easton, C. J.; Hutton, C. A. Synthesis of Each Stereoisomer of $\left[3-{ }^{2} \mathrm{H}_{1}\right]$ Phenylalanine and Evaluation of the Stereochemical Course of the Reaction of $(R)$-Phenylalanine with $(S)$ Phenylalanine Ammonia-Lyase. J. Chem. Soc. Perkin Trans. 1, 1994, 3545-3548.

(226) Viergutz, S.; Poppe, L.; Tomin, A.; Rétey, J. Mechanistic Investigation of Phenylalanine Ammonia Lyase by Using $N$-Methylated Phenylalanines. Helv. Chim. Acta 2003, 86, 3601-3612.

(227) Rowles, I.; Groenendaal, B.; Binay, B.; Malone, K. J.; Willies, S. C.; Turner, N. J. Engineering of Phenylalanine Ammonia Lyase from Rhodotorula graminis for the Enhanced Synthesis of Unnatural L-Amino Acids. Tetrahedron 2016, 72, 7343-7347.

(228) Ahmed, S. T.; Parmeggiani, F.; Weise, N. J.; Flitsch, S. L.; Turner, N. J. Chemoenzymatic Synthesis of Optically Pure L- and D-Biarylalanines through Biocatalytic Asymmetric Amination and Palladium-Catalyzed Arylation. ACS Catal. 2015, 5, 5410-5413. 
(229) Van Assema, F. B. J.; Sereinig, N. Method for Producing Optically Active Phenylalanine compounds from Cinnamic Acid Derivatives Employing Phenylalanine Ammonia Lyase Derived from Idiomarina loihiensis,. WO 2008031578 A1, 2008.

(230) Weise, N. J.; Ahmed, S. T.; Parmeggiani, F.; Siirola, E.; Pushpanath, A.; Schell, U.; Turner, N. J. Intensified Biocatalytic Production of Enantiomerically Pure Halophenylalanines from Acrylic Acids Using Ammonium Carbamate as the Ammonia Source. Catal. Sci. Technol. 2016, 6, 4086-4089.

(231) Parmeggiani, F.; Ahmed, S. T.; Weise, N. J.; Turner, N. J. Telescopic One-Pot Condensation-Hydroamination Strategy for the Synthesis of Optically Pure LPhenylalanines from Benzaldehydes. Tetrahedron 2016, 72, 7256-7262.

(232) D’Cunha, G. B.; Satyanarayan, V.; Nair, P. M. Novel Direct Synthesis of LPhenylalanine Methyl Ester by Using Rhodotorula glutinis Phenylalanine Ammonia Lyase in an Organic-Aqueous Biphasic System. Enzyme Microb. Technol. 1994, 16, 318 322.

(233) Hanson, K. R.; Havir, E. A.; Ressler, C. Phenylalanine Ammonia-Lyase: Enzymatic Conversion of 3-(1,4-Cyclohexadienyl)-L-Alanine to trans-3-(1,4Cyclohexadienyl)acrylic Acid. Biochemistry 1979, 18, 1431-1438.

(234) Skolaut, A.; Rétey, J. 1,4-Dihydro-L-Phenylalanine. Its Synthesis and Behavior in the Phenylalanine Ammonia-Lyase Reaction. Arch. Biochem. Biophys. 2001, 393, 187-191.

(235) Gloge, A.; Langer, B.; Poppe, L.; Rétey, J. The Behavior of Substrate Analogues and Secondary Deuterium Isotope Effects in the Phenylalanine Ammonia-Lyase Reaction. Arch. Biochem. Biophys. 1998, 359, 1-7.

(236) Paizs, C.; Katona, A.; Rétey, J. The Interaction of Heteroaryl-Acrylates and Alanines with Phenylalanine Ammonia-Lyase from Parsley. Chem. Eur. J. 2006, 12, 2739-2744.

(237) Paizs, C.; Toşa, M. I.; Bencze, L. C.; Brem, J.; Irimie, F. D.; Rétey, J. 2-Amino-3-(5Phenylfuran-2-yl)propionic Acids And 5-Phenylfuran-2-ylacrylic Acids Are Novel Substrates of Phenylalanine Ammonia-Lyase. Heterocycles 2010, 82, 1217-1228.

(238) Hashimoto, M.; Hatanaka, Y.; Nabeta, K. Novel Photoreactive Cinnamic Acid Analogues to Elucidate Phenylalanine Ammonia-Lyase. Bioorg. Med. Chem. Lett. 2000, 10, 24812483.

(239) Tian, Z.; Zhu, W.; Xu, Y.; Qian, X. An Unnatural Amino Acid Based Fluorescent Probe for Phenylalanine Ammonia Lyase. Org. Biomol. Chem. 2014, 12, 5818-5821.

(240) Lovelock, S. L.; Turner, N. J. Bacterial Anabaena variabilis Phenylalanine Ammonia Lyase: A Biocatalyst with Broad Substrate Specificity. Bioorg. Med. Chem. 2014, 22, $5555-5557$.

(241) Barron, C. C.; Sponagle, B. J. D.; Arivalagan, P.; D’Cunha, G. B. Optimization of Oligomeric Enzyme Activity in Ionic Liquids Using Rhodotorula glutinis Yeast Phenylalanine Ammonia Lyase. Enzyme Microb. Technol. 2017, 96, 151-156.

(242) Bartha-Vári, J. H.; Toşa, M. I.; Irimie, F. D.; Weiser, D.; Boros, Z.; Vértessy, B. G.; Paizs, C.; Poppe, L. Immobilization of Phenylalanine Ammonia-Lyase on Single-Walled Carbon Nanotubes for Stereoselective Biotransformations in Batch and Continuous-Flow Modes. ChemCatChem 2015, 7, 1122-1128.

(243) Bartha-Vári, J. H.; Bencze, L. C.; Bell, E.; Poppe, L.; Katona, G.; Irimie, F. D.; Paizs, C.; Toşa, M. I. Aminated Single-Walled Carbon Nanotubes as Carrier for Covalent Immobilization of Phenylalanine Ammonia-Lyase. Period. Polytechn. Chem. Eng. 2017, 61, 59-66. 
(244) Zhang, Z. Enantioselective Scavenging Using Homogenate of Rhodotorula graminis: A Facile Preparation of D-Amino Acid Derivatives in Enantiopure Form. Tetrahedron Lett. 2008, 49, 6468-6470.

(245) Shibatani, T.; Nishimura, N.; Nabe, K.; Kakimoto, T.; Chibata, I. Enzymatic Production of Urocanic Acid by Achromobacter liquidum. Appl. Microbiol. 1974, 27, 688-694.

(246) Zhu, L.; Zhou, L.; Huang, N.; Cui, W.; Liu, Z.; Xiao, K.; Zhou, Z. Efficient Preparation of Enantiopure D-Phenylalanine through Asymmetric Resolution Using Immobilized Phenylalanine Ammonia-Lyase from Rhodotorula glutinis $\mathrm{JN}-1$ in a Recirculating Packed-Bed Reactor. PLoS One 2014, 9, e108586.

(247) Ahmed, S. T.; Parmeggiani, F.; Weise, N. J.; Flitsch, S. L.; Turner, N. J. Synthesis of Enantiomerically Pure Ring-Substituted L-Pyridylalanines by Biocatalytic Hydroamination. Org. Lett. 2016, 18, 5468-5471.

(248) Ratnayake, N. D.; Liu, N.; Kuhn, L. A.; Walker, K. D. Ring-Substituted $\alpha$-Arylalanines for Probing Substituent Effects on the Isomerization Reaction Catalyzed by an Aminomutase. ACS Catal. 2014, 4, 3077-3090.

(249) Ratnayake, N. D.; Theisen, C.; Walter, T.; Walker, K. D. Whole-Cell Biocatalytic Production of Variously Substituted $\beta$-Aryl- and $\beta$-Heteroaryl- $\beta$-Amino Acids. $J$. Biotechnol. 2016, 217, 12-21.

(250) Gademann, K.; Hintermann, T.; Schreiber, J. V. $\beta$-Peptides: Twisting and Turning. Curr. Med. Chem. 1999, 6, 905-925.

(251) Steer, D. L.; Lew, R. A.; Perlmutter, P.; Smith, A. I.; Aguilar, M.-I. $\beta$-Amino Acids: Versatile Peptidomimetics. Curr. Med. Chem. 2002, 9, 811-822.

(252) Aguilar, M.-I.; Purcell, A. W.; Devi, R.; Lew, R.; Rossjohn, J.; Smith, A. I.; Perlmutter, P. $\beta$-Amino Acid-Containing Hybrid Peptides. New Opportunities in Peptidomimetics. Org. Biomol. Chem. 2007, 5, 2884-2890.

(253) Wu, B.; Szymański, W.; Wijma, H. J.; Crismaru, C. G.; De Wildeman, S.; Poelarends, G. J.; Feringa, B. L.; Janssen, D. B. Engineering of an Enantioselective Tyrosine Aminomutase by Mutation of a Single Active Site Residue in Phenylalanine Aminomutase. Chem. Commun. 2010, 46, 8157-8159.

(254) Klettke, K. L.; Sanyal, S.; Mutatu, W.; Walker, K. D. $\beta$-Styryl- and $\beta$-Aryl- $\beta$-Alanine Products of Phenylalanine Aminomutase Catalysis. J. Am. Chem. Soc. 2007, 129, 69886989.

(255) Cox, B. M.; Bilsborrow, J. B.; Walker, K. D. Enhanced Conversion of Racemic $\alpha-$ Arylalanines to $(R)-\beta$-Arylalanines by Coupled Racemase/Aminomutase Catalysis. $J$. Org. Chem. 2009, 74, 6953-6959.

(256) Walker, K. D.; Cox, B. M. Process for the Preparation of $(R)$-Beta-Arylalanines by Coupled Racemase/Aminomutase Catalysis. US 20100285540, 2010.

(257) Wanninayake, U.; DePorre, Y.; Ondari, M.; Walker, K. D. (S)-Styryl- $\alpha$-Alanine Used to Probe the Intermolecular Mechanism of an Intramolecular MIO-Aminomutase. Biochemistry 2011, 50, 10082-10090.

(258) Wanninayake, U.; Walker, K. D. Assessing the Deamination Rate of a Covalent Aminomutase Adduct by Burst Phase Analysis. Biochemistry 2012, 51, 5226-5228.

(259) Hidesaki, T. Phenylalanine Aminomutase with Selectivity for $S$-Form, DNA Encoding the Same, Method for Producing $(S)$ - $\beta$-Phenylalanine and Method for Producing $(S)-\beta$ Phenylalanine Analogs. WO 2008129873, 2008.

(260) Hidesaki, T. Method for Producing $\beta$-Amino Acid. WO 2009044531, 2009. 
(261) Varga, A.; Bánóczi, G.; Nagy, B.; Bencze, L. C.; Toşa, M. I.; Gellért, Á.; Irimie, F. D.; Rétey, J.; Poppe, L.; Paizs, C. Influence of the Aromatic Moiety in $\alpha$ - and $\beta$-Arylalanines on Their Biotransformation with Phenylalanine 2,3-Aminomutase from Pantoea agglomerans. RSC Adv. 2016, 6, 56412-56420.

(262) Wu, B.; Szymański, W.; De Wildeman, S.; Poelarends, G. J.; Feringa, B. L.; Janssen, D. B. Efficient Tandem Biocatalytic Process for the Kinetic Resolution of Aromatic $\beta$ Amino Acids. Adv. Synth. Catal. 2010, 352, 1409-1412.

(263) Wu, B.; Szymański, W.; Wietzes, P.; De Wildeman, S.; Poelarends, G. J.; Feringa, B. L.; Janssen, D. B. Enzymatic Synthesis of Enantiopure $\alpha$ - and $\beta$-Amino Acids by Phenylalanine Aminomutase-Catalysed Amination of Cinnamic Acid Derivatives. ChemBioChem 2009, 10, 338-344.

(264) Szymański, W.; Wu, B.; Weiner, B.; de Wildeman, S.; Feringa, B. L.; Janssen, D. B. Phenylalanine Aminomutase-Catalyzed Addition of Ammonia to Substituted Cinnamic Acids: A Route to Enantiopure $\alpha$ - and $\beta$-Amino Acids. J. Org. Chem. 2009, 74, 91529157.

(265) Verkuijl, B. J. V; Szymański, W.; Wu, B.; Minnaard, A. J.; Janssen, D. B.; de Vries, J. G.; Feringa, B. L. Enantiomerically Pure $\beta$-Phenylalanine Analogues from $\alpha-\beta$ Phenylalanine Mixtures in a Single Reactive Extraction Step. Chem. Commun. 2010, 46, 901-903.

(266) Heberling, M. M.; Masman, M. F.; Bartsch, S.; Wybenga, G. G.; Dijkstra, B. W.; Marrink, S. J.; Janssen, D. B. Ironing out Their Differences: Dissecting the Structural Determinants of a Phenylalanine Aminomutase and Ammonia Lyase. ACS Chem. Biol. 2015, 10, 989-997.

(267) Williams, J. S.; Thomas, M.; Clarke, D. J. The Gene stlA Encodes a Phenylalanine Ammonia-Lyase That Is Involved in the Production of a Stilbene Antibiotic in Photorhabdus luminescens TT01. Microbiology 2005, 151 (Pt 8), 2543-2550.

(268) Weise, N. J.; Parmeggiani, F.; Ahmed, S. T.; Turner, N. J. The Bacterial Ammonia Lyase EncP: A Tunable Biocatalyst for the Synthesis of Unnatural Amino Acids. J. Am. Chem. Soc. 2015, 137, 12977-12983.

(269) Segala, M.; Takahata, Y.; Chong, D. P. Geometry, Solvent, and Polar Effects on the Relationship between Calculated Core-Electron Binding Energy Shifts $(\triangle \mathrm{CEBE})$ and Hammett Substituent ( $\sigma$ ) Constants. J. Mol. Struct. THEOCHEM 2006, 758, 61-69.

(270) Paizs, C.; Katona, A.; Rétey, J. Chemoenzymatic One-Pot Synthesis of Enantiopure LArylalanines from Arylaldehydes. Eur. J. Org. Chem. 2006, 1113-1116.

(271) De Lange, B.; Hyett, D. J.; Maas, P. J. D.; Mink, D.; Van Assema, F. B. J.; Sereinig, N.; De Vries, A. H. M.; De Vries, J. G. Asymmetric Synthesis of $(S)$-2-Indolinecarboxylic Acid by Combining Biocatalysis and Homogeneous Catalysis. ChemCatChem 2011, 3, 289-292.

(272) Parmeggiani, F.; Weise, N. J.; Ahmed, S. T.; Turner, N. J. Chemoenzymatic Synthesis of (S)-1,2,3,4-Tetrahydroisoquinoline-3-Carboxylic Acid by PAL-Mediated Amination and Pictet-Spengler Cyclization. In Practical Methods for Biocatalysis and Biotransformations 3, 2016; pp. 243-246.

(273) Roughley, S. D.; Jordan, A. M. The Medicinal Chemist's Toolbox: An Analysis of Reactions Used in the Pursuit of Drug Candidates. J. Med. Chem. 2011, 54, 3451-3479.

(274) Nielsen, D. R.; McKenna, R. Microbial Conversion of Glucose to Styrene and Its Derivatives. WO 2012122333 A1, 2012. 
(275) Busto, E.; Simon, R. C.; Kroutil, W. Vinylation of Unprotected Phenols Using a Biocatalytic System. Angew. Chem. Int. Ed. 2015, 54, 10899-10902.

(276) Zhou, Y.; Wu, S.; Li, Z. Cascade Biocatalysis for Sustainable Asymmetric Synthesis: From Biobased L-Phenylalanine to High-Value Chiral Chemicals. Angew. Chem. Int. Ed. 2016, 55, 11647-11650.

(277) Katsuyama, Y.; Funa, N.; Horinouchi, S. Precursor-Directed Biosynthesis of Stilbene Methyl Ethers in Escherichia coli. Biotechnol. J. 2007, 2, 1286-1293.

(278) Li, M.; Kildegaard, K. R.; Chen, Y.; Rodriguez, A.; Borodina, I.; Nielsen, J. De novo Production of Resveratrol from Glucose or Ethanol by Engineered Saccharomyces cerevisiae. Metab. Eng. 2015, 32, 1-11.

(279) Li, M.; Schneider, K.; Kristensen, M.; Borodina, I.; Nielsen, J. Engineering Yeast for High-Level Production of Stilbenoid Antioxidants. Sci. Rep. 2016, 6, 36827.

(280) Ro, D. K.; Douglas, C. J. Reconstitution of the Entry Point of Plant Phenylpropanoid Metabolism in Yeast (Saccharomyces cerevisiae): Implications for Control of Metabolic Flux into the Phenylpropanoid Pathway. J. Biol. Chem. 2004, 279, 2600-2607.

(281) Miyahisa, I.; Funa, N.; Ohnishi, Y.; Martens, S.; Moriguchi, T.; Horinouchi, S. Combinatorial Biosynthesis of Flavones and Flavonols in Escherichia coli. Appl. Microbiol. Biotechnol. 2006, 71, 53-58.

(282) Trantas, E.; Panopoulos, N.; Ververidis, F. Metabolic Engineering of the Complete Pathway Leading to Heterologous Biosynthesis of Various Flavonoids and Stilbenoids in Saccharomyces cerevisiae. Metab. Eng. 2009, 11, 355-366.

(283) Jendresen, C. B.; Stahlhut, S. G.; Li, M.; Gaspar, P.; Siedler, S.; Förster, J.; Maury, J.; Borodina, I.; Nielsen, A. T. Highly Active and Specific Tyrosine Ammonia-Lyases from Diverse Origins Enable Enhanced Production of Aromatic Compounds in Bacteria and Saccharomyces cerevisiae. Appl. Environ. Microbiol. 2015, 81, 4458-4476.

(284) Cantor, J. R.; Panayiotou, V.; Agnello, G.; Georgiou, G.; Stone, E. M. Engineering Reduced-Immunogenicity Enzymes for Amino Acid Depletion Therapy in Cancer. Methods Enzymol. 2012, 502, 291-319.

(285) Fu, Y. M.; Yu, Z. X.; Pelayo, B. A.; Ferrans, V. J.; Meadows, G. G. Focal Adhesion Kinase-Dependent Apoptosis of Melanoma Induced by Tyrosine and Phenylalanine Deficiency. Cancer Res. 1999, 59, 758-765.

(286) Pelayo, B. A.; Fu, Y. M.; Meadows, G. G. Inhibition of B16BL6 Melanoma Invasion by Tyrosine and Phenylalanine Deprivation Is Associated with Decreased Secretion of Plasminogen Activators and Increased Plasminogen Activator Inhibitors. Clin. Exp. Metastasis 1999, 17, 841-848.

(287) Kovács, K.; Bánóczi, G.; Varga, A.; Szabó, I.; Holczinger, A.; Hornyánszky, G.; Zagyva, I.; Paizs, C.; Vértessy, B. G.; Poppe, L. Expression and Properties of the Highly Alkalophilic Phenylalanine Ammonia-Lyase of Thermophilic Rubrobacter xylanophilus. PLoS One 2014, 9, e85943.

(288) Abell, C. W.; Stith, W. J.; Hodgins, D. S. The Effects of Phenylalanine Ammonia-Lyase on Leukemic Lymphocytes in vitro. Cancer Res. 1972, 32, 285-290.

(289) Stith, W. J.; Hodgins, D. S.; Abell, C. W. Effects of Phenylalanine Ammonia-Lyase and Phenylalanine Deprivation on Murine Leukemic Lymphoblasts in vitro. Cancer Res. 1973, 33, 966-971.

(290) Abell, C. W.; Hodgins, D. S.; Stith, W. J. An in vivo Evaluation of the Chemotherapeutic Potency of Phenylalanine Ammonia-Lyase. Cancer Res. 1973, 33, 2529-2532. 
(291) Roberts, J.; Schmid, F. A.; Rosenfeld, H. J. Biological and Antineoplastic Effects of Enzyme-Medicated in vivo Deletion of L-Glutamine, L-Trytophan, and L-Histidine. Cancer Treat. Rep. 1979, 63, 1045-1054.

(292) Jack, G.; Wilbin, C.; McMahon, P. The Effect of Histidine Ammonia Lyase on Some Murine Tumours. Leuk. Res. 1983, 7, 421-429.

(293) Shen, R.; Fritz, R.; Abell, C. Biochemical Properties and Immunogenicity of LPhenylalanine Ammonia-Lyase: Effects on Tumor-Bearing Mice. Cancer Treat. Rep. 1979, 63, 1063-1068.

(294) Sethuraman, N.; Roberts, J.; Macallister, T. Cloning of Corynebacteriaceae Histidine Ammonia Lyase and Therapeutic Uses. WO 2001079469, 2001.

(295) Sethuraman, N.; Roberts, J.; Macallister, T. Cloning, Overexpression and Therapeutic Use of Bioactive Histidine Ammonia Lyase. US 6939541, 2005.

(296) Vellard, M. C.; Fitzpatrick, P. A.; Kakkis, E. D.; Wendt, D. J.; Muthalif, M.; Bell, S. M.; Okhamafe, A. O. Compositions of Prokaryotic Phenylalanine Ammonia-Lyase and Methods of Treating Cancer Using Compositions Thereof. WO 2009025760, 2009.

(297) Babich, O. O.; Pokrovsky, V. S.; Anisimova, N. Y.; Sokolov, N. N.; Prosekov, A. Y. Recombinant L-Phenylalanine Ammonia Lyase from Rhodosporidium toruloides as a Potential Anticancer Agent. Biotechnol. Appl. Biochem. 2013, 60, 316-322.

(298) Gámez, A.; Sarkissian, C. N.; Wang, L.; Kim, W.; Straub, M.; Patch, M. G.; Chen, L.; Striepeke, S.; Fitzpatrick, P.; Lemontt, J. F.; O'Neill, C.; Scriver, C. R.; Stevens, R. C. Development of Pegylated Forms of Recombinant Rhodosporidium toruloides Phenylalanine Ammonia-Lyase for the Treatment of Classical Phenylketonuria. Mol. Ther. 2005, 11, 986-989.

(299) Sarkissian, C. N.; Kang, T. S.; Gámez, A.; Scriver, C. R.; Stevens, R. C. Evaluation of Orally Administered PEGylated Phenylalanine Ammonia Lyase in Mice for the Treatment of Phenylketonuria. Mol. Genet. Metab. 2011, 104, 249-254.

(300) Rossi, L.; Pierigè, F.; Carducci, C.; Gabucci, C.; Pascucci, T.; Canonico, B.; Bell, S. M.; Fitzpatrick, P. A.; Leuzzi, V.; Magnani, M. Erythrocyte-Mediated Delivery of Phenylalanine Ammonia Lyase for the Treatment of Phenylketonuria in BTBR-Pah ${ }^{\text {enu } 2}$ Mice. J. Control. Release 2014, 194, 37-44.

(301) Bélanger-Quintana, A.; Burlina, A.; Harding, C. O.; Muntau, A. C. Up to Date Knowledge on Different Treatment Strategies for Phenylketonuria. Mol. Genet. Metab. 2011, 104, S19-S25.

(302) Ikeda, K.; Schiltz, E.; Fujii, T.; Takahashi, M.; Mitsui, K.; Kodera, Y.; Matsushima, A.; Inada, Y.; Schulz, G. E.; Nishimura, H. Phenylalanine Ammonia-Lyase Modified with Polyethylene Glycol: Potential Therapeutic Agent for Phenylketonuria. Amino Acids 2005, 29, 283-287.

(303) Huisman, G. W.; Agard, N. J.; Elgart, D.; Zhang, X. Engineered Tyrosine Ammonia Lyase. WO 2015161019 A1, 2015.

(304) Castañeda, M. T.; Adachi, O.; Hours, R. A. Reduction of L-Phenylalanine in Protein Hydrolysates Using L-Phenylalanine Ammonia-Lyase from Rhodosporidium toruloides. J. Ind. Microbiol. Biotechnol. 2015, 42, 1299-1307.

(305) Sarkissian, C. N.; Gámez, A. Phenylalanine Ammonia Lyase, Enzyme Substitution Therapy for Phenylketonuria, Where Are We Now? Mol. Genet. Metab. 2005, 86, S22S26. 
(306) Kang, T. S.; Wang, L.; Sarkissian, C. N.; Gámez, A.; Scriver, C. R.; Stevens, R. C. Converting an Injectable Protein Therapeutic into an Oral Form: Phenylalanine Ammonia Lyase for Phenylketonuria. Mol. Genet. Metab. 2010, 99, 4-9.

(307) Longo, N.; Harding, C. O.; Burton, B. K.; Grange, D. K.; Vockley, J.; Wasserstein, M.; Rice, G. M.; Dorenbaum, A.; Neuenburg, J. K.; Musson, D. G.; Gu, Z.; Sile, S. SingleDose, Subcutaneous Recombinant Phenylalanine Ammonia Lyase Conjugated with Polyethylene Glycol in Adult Patients with Phenylketonuria: an Open-Label, Multicentre, Phase 1 Dose-Escalation Trial. Lancet 2014, 384, 37-44.

(308) Jaliani, H. Z.; Farajnia, S.; Mohammadi, S. A.; Barzegar, A.; Talebi, S. Engineering and Kinetic Stabilization of the Therapeutic Enzyme Anabaena variabilis Phenylalanine Ammonia Lyase. Appl. Biochem. Biotechnol. 2013, 171, 1805-1818.

(309) Liu, J.; Jia, X.; Zhang, J.; Xiang, H.; Hu, W.; Zhou, Y. Study on a Novel Strategy to Treatment of Phenylketonuria. Artif. Cells, Blood Substit., Immobil. Biotechnol. 2009, 30, 243-257.

(310) Zhang, Y.; Jia, X.; Wang, L.; Liu, J.; Ma, G. Preparation of Ca-Alginate Microparticles and Its Application for Phenylketonuria Oral Therapy. Ind. Eng. Chem. Res. 2011, 50, 4106-4112.

(311) Bourget, L.; Chang, T. M. S. Phenylalanine Ammonia-Lyase Immobilized in Semipermeable Microcapsules for Enzyme Replacement in Phenylketonuria. FEBS Lett. 1985, 180, 5-8.

(312) Shah, R. M.; D'mello, A. P. Stabilization of Phenylalanine Ammonia Lyase against Organic Solvent Mediated Deactivation. Int. J. Pharm. 2007, 331, 107-115.

(313) Shah, R. M.; D'mello, A. P. Strategies to Maximize the Encapsulation Efficiency of Phenylalanine Ammonia Lyase in Microcapsules. Int. J. Pharm. 2008, 356, 61-68.

(314) Wang, L.; Gamez, A.; Sarkissian, C. N.; Straub, M.; Patch, M. G.; Won Han, G.; Striepeke, S.; Fitzpatrick, P.; Scriver, C. R.; Stevens, R. C. Structure-Based Chemical Modification Strategy for Enzyme Replacement Treatment of Phenylketonuria. Mol. Genet. Metab. 2005, 86, 134-140.

(315) MacDonald, C. B. J.; Tobin, A. M. F.; Morrison, A. A. E.; Tait, M. E.; D’Cunha, A. G. B.; A, S. L. M. Generation of a Highly Stable Reusable Biocatalyst by Entrapment of an Oligomeric Enzyme in Ultra-Large-Pore Mesoporous Silica. Aust. J. Chem. 2015, 68, 396-400.

(316) Hosta-Rigau, L.; York-Duran, M. J.; Kang, T. S.; Städler, B. Extracellular Microreactor for the Depletion of Phenylalanine Toward Phenylketonuria Treatment. Adv. Funct. Mater. 2015, 25, 3860-3869.

(317) Cui, J. D.; Zhang, S.; Sun, L. M. Cross-Linked Enzyme Aggregates of Phenylalanine Ammonia Lyase: Novel Biocatalysts for Synthesis of L-Phenylalanine. Appl. Biochem. Biotechnol. 2012, 167, 835-844.

(318) Cui, J. D.; Li, L. L.; Zhao, Y. M. Simple Technique for Preparing Stable and Recyclable Cross-Linked Enzyme Aggregates with Crude-Pored Microspherical Silica Core. Ind. Eng. Chem. Res. 2014, 53, 16176-16182.

(319) Zhu, L.; Zhou, L.; Cui, W.; Liu, Z.; Zhou, Z. Mechanism-Based Site-Directed Mutagenesis to Shift the Optimum $\mathrm{pH}$ of the Phenylalanine Ammonia-Lyase from Rhodotorula glutinis JN-1. Biotechnol. Reports 2014, 3, 21-26.

(320) Eigtved, P.; Groth Clausen, I. Stabilised Phenylalanine Ammonia Lyase. US 5753487 A, 1998. 
(321) Huisman, G. W.; Agard, N. J.; Mijts, B.; Vroom, J.; Zhang, X. Engineered Phenylalanine Ammonia Lyase Polypeptides. WO 2014172541 A3, 2015.

(322) Noel, J. P.-; Louie, G. V; Bowman, M. E.; Moore, B. S.; Moffitt, M. C. Substrate Switched Ammonia Lyases and Mutases. US 20090011400, 2009.

$\underline{\text { Table of Contents Graphic }}$

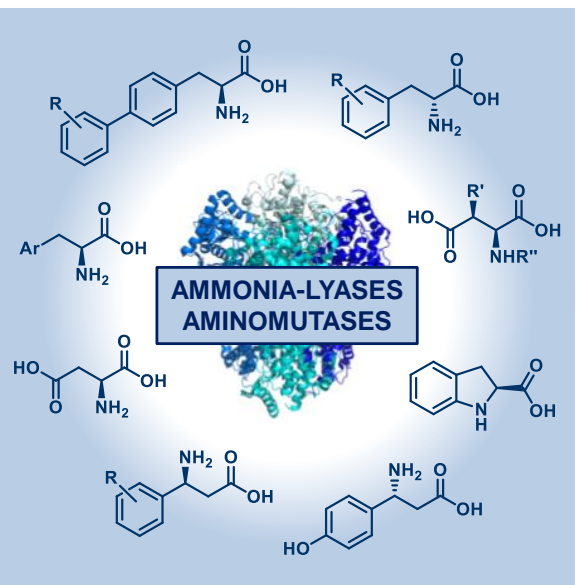

